\title{
P2Y6 receptor signaling in natural killer cells impairs insulin sensitivity in obesity
}

Anna Sieben ${ }^{1,2}$, Anne Christine Hausen ${ }^{1}$, Paul Klemm ${ }^{1}$, Alexander Jais ${ }^{1,3}$, Christian Heilinger ${ }^{1}$, Jens Alber ${ }^{1}$, Kat Folz-Donahue ${ }^{4}$, Lena Schumacher ${ }^{4}$, Janine Altmüller ${ }^{5}$, Marek Franitza ${ }^{5}$, Patrick Giavalisco ${ }^{6}$, Yvonne Hinze ${ }^{6}$, Susanne Brodesser ${ }^{7}$, Christian Kukat $^{4}$, Sebastian Theurich ${ }^{8,9}$, Jens C. Brüning ${ }^{1,2,10, \# \text {. }}$

'Max Planck Institute for Metabolism Research, AG Neuronal Control of Metabolism, Gleueler Straße 50, 50931 Cologne, Germany

${ }^{2}$ Center for Endocrinology, Diabetes and Preventive Medicine (CEDP), University Hospital Cologne, Kerpener Straße 26, 50924 Cologne, Germany

${ }^{3} \mathrm{HI}-\mathrm{MAG}$ Helmholtz Institute for Metabolic, Obesity and Vascular Research, AG Diet-Induced Metabolic Alterations, Philipp-Rosenthal-Str. 27, 04103 Leipzig, Germany

${ }^{4}$ Max Planck Institute for Biology of Ageing, FACS \& Imaging Core Facility, Joseph-StelzmannStr. 9B, 50931 Cologne, Germany

${ }^{5}$ Cologne Center for Genomics (CCG), University of Cologne, Next-Generation-Sequencing Core Facility, Weyertal 115b, 50931 Cologne, Germany

${ }^{6}$ Max Planck Institute for Biology of Ageing, Metabolomics Core Facility, Joseph-StelzmannStr. 9b, 50931 Cologne, Germany

${ }^{7}$ CECAD Research Center, Lipidomics/Metabolomics Facility, Joseph-Stelzmann-Str. 26, 50931 Cologne, Germany

${ }^{8}$ Department of Medicine III, University Hospital, LMU Munich, Munich, Germany

${ }^{9}$ Cancer and Immunometabolism Research Group, Gene Center LMU, Feodor-LynenStr. 25, 81377 Munich, Germany

${ }^{10}$ Lead contact

\#Address correspondence to:

Jens C. Brüning, M.D.

Max Planck Institute for Metabolism Research

Gleueler Str. 50

50931 Cologne, Germany

Phone: +49-221 4726202

Fax: +49-221 4726203

e-mail: bruening@sf.mpg.de 
53 Natural killer (NK) cells contribute to the development of obesity-associated insulin

54 resistance and have previously been shown to up-regulate the expression of the P2Y

55 purinoreceptor 6 (P2Y6R) upon high fat diet (HFD)-induced obesity. Here, we reveal

56 that NK cell-specific inactivation of the P2Y6R gene improves insulin sensitivity in

57 obese mice and reduces the expression of chemokines in adipose tissue infiltrating

58 NK-cells. Obese mice lacking P2Y6R specifically in NK cells exhibited a reduction in

59 adipose tissue inflammation, exhibited improved insulin-stimulated suppression of

60 lipolysis in adipose tissue and a reduction in hepatic glucose production, leading to an

61 overall improvement of systemic insulin sensitivity. In contrast, myeloid lineage specific

62 P2Y6R inactivation does not affect energy or glucose homeostasis in obesity.

63 Collectively, we show that P2Y6R signaling in NK cells contributes to the development

64 of obesity-associated insulin resistance and thus might be a future target for the

65 treatment of obesity-associated insulin resistance and type 2 diabetes.

66 
68 More than 1.9 billion adults were overweight in 2016. Of these, over 650 million adults

69 were obese $(\mathrm{WHO}, 2021)$, reflecting a slow but dangerous global pandemic that started 70 more than 40 years ago (WHO, 2021). Obesity not only increases the risk of co-

71 morbidities such as cardiovascular diseases, cancer and diabetes mellitus (Guh et al.,

72 2009) but also has profound effects on the immune system (De Heredia et al., 2012).

73 Obesity is associated with a chronic low-grade inflammation, termed metaflammation

74 (De Heredia et al., 2012), which is reflected in an increased number of macrophages

75 (Weisberg et al., 2003), T cells and natural killer (NK) cells (Theurich et al., 2017)

76 infiltrating the adipose tissue and a rise in pro-inflammatory molecules in tissues and

77 blood (Xu et al., 2003; Schmidt et al., 2015).

78 Nucleotides such as adenosine and uridine are critical building blocks of the

79 genetic code and at the same time key regulators of inflammation (Idzko et al., 2014).

80 Uridine acts as a potent anti-inflammatory molecule during lung inflammation

81 (Evaldsson et al., 2007) and pulmonary fibrosis (Cicko et al., 2015). Here, it affects

82 adhesion between leukocytes and endothelium in a lung inflammation model

83 (Uppugunduri and Gautam, 2004). Plasma uridine concentrations are regulated by

84 fasting and refeeding (Deng et al., 2017), controlled by the circadian rhythm (El Kouni

85 et al., 1990) and exercise (Yamamoto et al., 1997) and positively correlate with insulin

86 resistance and plasma insulin levels (Hamada et al., 2007). Uridine impairs insulin

87 sensitivity and glucose tolerance in mice (Urasaki et al., 2014), has an impact on liver

88 lipid metabolism (Urasaki et al., 2016; Le et al., 2013) as well as is involved in glycogen

89 metabolism (Haugaard et al., 1977).

Moreover, uridine serves as precursor for the synthesis of the nucleotide uridine

91 diphosphate (UDP). Coupled to glucose, this nucleotide is a critical component in the

92 synthesis pathway of glycogen (Berg et al., 2002). At the same time, UDP acts as an

93 extracellular signaling molecule after being released from apoptotic and necrotic cells

94 and serves as danger and find-me signal in order to attract immune cells to the site of

95 inflammation (Elliott et al., 2009). On the other hand, a controlled way of nucleotide

96 release has been reported (Lazarowski et al., 2011). Here, the extracellular nucleotide

97 adenosine triphosphate (ATP) can act as an initiator or terminator of immune

98 responses to induce different effects on immune cells depending on the pattern of P2

99 receptors engaged, the duration of the stimulus and its concentration in the

100 extracellular milieu (Gorini et al., 2010). 
The extracellular nucleotide UDP binds to P2Y6 receptors (P2Y6R) which

102 belong to the family of G-protein coupled receptors. The activation of Gq-coupled

103 P2Y6Rs results in a rise of intracellular calcium concentration leading to an altered

104 activity of kinases such as protein kinase C (PKC), mitogen-activated protein kinase

105 (MAPK) and extracellular signal-regulated kinase (ERK), to changes in transcription

106 and to the release of signaling molecules (Kim et al., 2003; Li et al., 2014; Zhang et al.,

107 2011). In the context of glucose metabolism and obesity it has been shown that UDP-

108 P2Y6R signaling regulates feeding via hypothalamic agouti-related protein (AgRP)

109 neurons (Steculorum et al., 2015) and that P2Y6R action in AgRP neurons contributes

110 to HFD-induced deterioration of insulin sensitivity (Steculorum et al., 2017).

111 Investigations in peripheral organs revealed that adipocyte-specific deletion of P2Y6R

112 protects mice from diet-induced obesity, improves glucose tolerance and insulin

113 sensitivity and reduces systemic inflammation (Jain et al., 2020). In contrast to that,

114 P2Y6R deletion in skeletal muscle resulted in impaired glucose homeostasis (Jain et

115 al., 2020).

116 P2Y6Rs are also expressed in a variety of immune cells, such as macrophages

117 (Bar et al., 2008; Lattin et al., 2008), T cells (Giannattasio et al., 2011) and microglia

118 (Idzko et al., 2004, Kim et al., 2011). Moreover, expression of P2Y6R is upregulated in

119 NK cells isolated from adipose tissue of mice fed a HFD when compared to lean mice

120 on a control diet (Theurich et al., 2017). NK cells are part of the innate immune system

121 responsible for the recognition and elimination of virus-infected and cancerous cells

122 (Vivier et al., 2008). They are able to destroy target cells via the release and joined

123 action of the cytotoxic mediators perforin and granzyme (Pardo et al., 2002) or through

124 the exocytosis of cytokines such as interferon gamma (IFNY) and tumor necrosis factor

125 alpha (TNFa) (Girart et al., 2007; Wang et al., 2012; Jewett et al., 1996). NK cell activity

126 is controlled by the orchestration of activating and inactivating receptors (Bakker et al.,

127 2000; Lanier, 2008) as well as by cytokines such as interleukin (IL) 2 and 15 (Zwirner

128 and Domaica, 2010). Obesity induces lipid accumulation in NK cells, leading to

129 cytotoxic dysfunction, impaired trafficking and reduced anti-tumor responses (Michelet

130 et al., 2018). NK cells derived from adipose tissue of obese mice show an activated

131 immune phenotype and release cytokines that trigger the differentiation and infiltration

132 of macrophages, finally resulting in increased inflammation and promotion of insulin

133 resistance (Wensveen et al., 2015; Lee et al., 2016). The majority of macrophages

134 infiltrating the adipose tissue of obese animals and humans are arranged around dead 
135 adipocytes, forming characteristic crown-like structures (CLS) (Murano et al., 2008;

136 Cinti et al., 2005). These necrotic adipocytes might release UDP as danger signal

137 leading to an increased infiltration or local proliferation of immune cells, thus promoting

138 metaflammation and development of insulin resistance.

139 Given the improvement of insulin action in conventional P2Y6R knockout mice

140 (Steculorum et al., 2017; Jain et al., 2020), and the role of P2Y6R-signaling in immune

141 cells, we aimed to investigate the role of this receptor in NK cells and macrophages in

142 the development of obesity-associated metaflammation and insulin resistance. To this

143 end, we generated mice lacking P2Y6R specifically in these immune compartments.

144 While ablation of P2Y6R in myeloid lineage cells had no effect on metaflammation and

145 obesity-associated insulin resistance, obese mice lacking P2Y6R specifically in NK

146 cells exhibited a reduction in adipose tissue inflammation, exhibited improved insulin-

147 stimulated suppression of lipolysis in adipose tissue and a reduction in hepatic glucose

148 production, leading to an overall improvement of systemic insulin sensitivity.

149 Collectively, these experiments extend the tissue-specific actions of P2Y6R signaling

150 in obesity, further characterizing P2Y6R inhibition as a novel target for metabolic

151 diseases. 


\section{Results}

153 Deletion of the P2Y6R gene in NK cells does not protect from HFD-induced 154 obesity

155 Among other genes, P2Y6R expression contributes to the discrimination of NK cells 156 from lean and obese mice (Theurich et al., 2017). Furthermore, it has been reported

157 that circulating uridine levels are increased in obesity (Steculorum et al., 2015; Deng 158 et al., 2017). To further define potential changes in uridine and UDP in different 159 organismal compartments upon development of HFD-induced obesity and insulin 160 resistance, we compared uridine and UDP levels in plasma, liver, adipocytes and the 161 adipose tissue stromal vascular fraction (SVF) of mice that had been kept on normal 162 chow diet (NCD) or had been exposed to HFD-feeding for 6 or 12 weeks. This analysis 163 revealed increased levels of uridine in plasma of obese compared to lean mice after 16412 weeks of HFD feeding (Suppl. Fig. S1A), while uridine levels in liver and SVF 165 increased already at 6 weeks of HFD feeding and remained elevated until 12 weeks of 166 HFD feeding (Suppl. Fig. S1B, C), while adipocyte uridine concentration increased 167 after 6 weeks of HFD feeding, but reached comparable levels to NCD-fed mice after 16812 weeks (Suppl. Fig. S1D). While UDP levels in plasma paralleled the increase of 169 circulating uridine 12 weeks after HFD-feeding (Suppl. Fig. S1E) as well as the 170 increases in adipose tissue SVF 6 and 12 weeks after high fat diet feeding (Suppl. Fig. 171 S1G), hepatic UDP levels remained unaltered upon HFD feeding (Suppl. Fig. S1F), 172 and adipocyte UDP levels increased only after prolonged HFD feeding (Suppl. Fig. $173 \mathrm{~S} 1 \mathrm{H})$. These experiments indicate a coordinated regulation of uridine and UDP 174 concentrations in plasma and SVF, while UDP synthesis in liver and adipocytes does 175 not appear to be directly linked to changes in local uridine concentrations. 
A

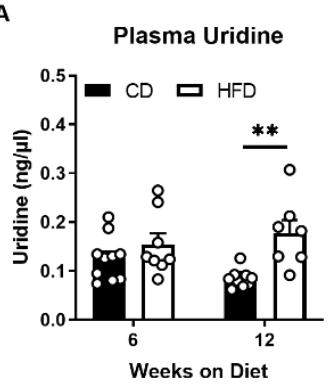

$\mathrm{E}$

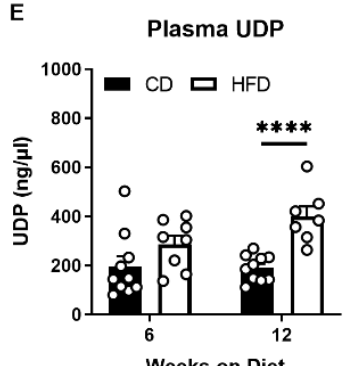

B
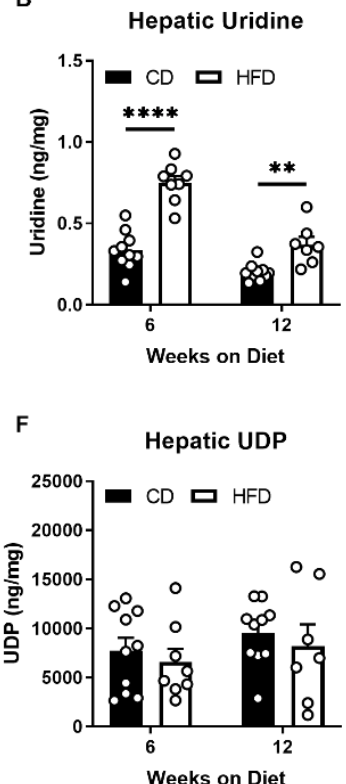
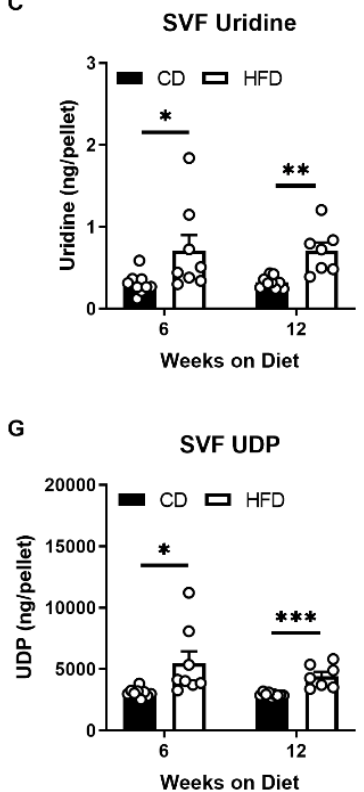

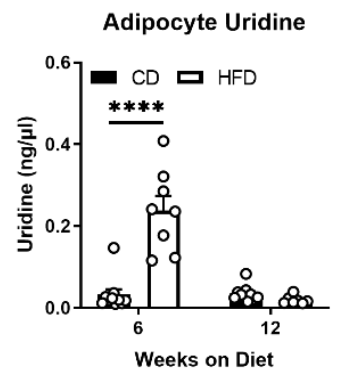

H

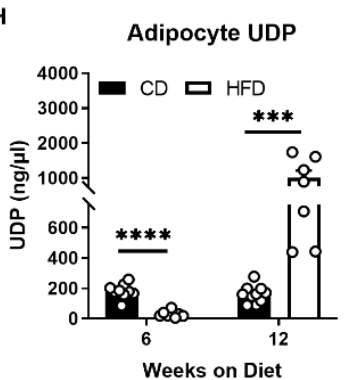

Fig. S1: Uridine and UDP levels are increased upon HFD feeding. Two cohorts of C57BI6 mice were either fed a normal chow diet (NCD) or HFD for 6 (NCD, n=10; HFD, n=8) or 12 (NCD, n=10; HFD, n=7) weeks. Uridine and UDP levels were determined in plasma (A, E), liver (B, F), cells of the stromal vascular fraction (SVF) $(\mathbf{C}, \mathbf{G})$ and in adipocytes (D, H). Statistics: Unpaired two-tailed t-test, ${ }^{*} \mathrm{p} \leq 0.05$, ${ }^{* *} p \leq 0.01,{ }^{* * *} p \leq 0.001,{ }^{* * *} p \leq 0.0001$. Data are represented as mean $\pm S E M$. $\circ$ denotes individual mice.

In light of the coordinated regulation of uridine and UDP in plasma and adipose

179 tissue SVF, compartments which directly interact with immune cells, we aimed to 180 investigate the impact of P2Y6R signaling in NK cells on body weight development,

181 glucose metabolism, and obesity-associated metaflammation in vivo. In order to 182 specifically delete the P2Y6R in NK cells, we crossed mice carrying a loxP-flanked 183 P2Y6R gene (Jain et al., 2020) with Ncr1-Cre mice (Eckelhardt et al., 2011). PCR 184 analysis of DNA isolated from NK cells of control (P2Y6 $\left.6^{f / f l}\right)$ or NK cell-specific P2Y6185 deleted (P2Y6 $\left.{ }^{\Delta N c 1}\right)$ mice confirmed successful and specific disruption of P2Y6R 186 expression in NK cells (Suppl. Fig. S2).

187 Starting at the age of 6 weeks, $\mathrm{P}^{2} \mathrm{Y}^{\Delta \mathrm{Ncr} 1}$ mice and their respective littermate controls 188 were fed a HFD or control diet (CD). HFD feeding did not result in any differences in 189 body weight between genotypes in male mice (Fig.1A), while female P2Y6 ${ }^{\Delta N c 1}$ mice 190 exhibited a mild protection from HFD-induced weight gain upon prolonged HFD191 feeding (Suppl. Fig. S3A). Consistent with unaltered body weight development, the 192 relative amount of lean and fat mass of male mice after 15 weeks on HFD was not 193 different between genotypes (Fig. 1B). In line with this, male P2Y6 ${ }^{\Delta \mathrm{Ncr} 1}$ mice did not 194 exhibit any differences in food intake (Suppl. Fig. S4A), water consumption (Suppl. Fig. 
195 S4B), energy expenditure (Fig. S4C) or locomotor activity (Fig. S4D) compared with

196 control mice on HFD. However, after 14 weeks of HFD feeding we could observe a

197 slight reduction in liver weights in male (Fig. 1C) and female (Suppl. Fig. S3B)

$198 \mathrm{P} 2 \mathrm{Y}^{\Delta \mathrm{N} c r 1}$ mice, as well as a decrease in fasted leptin levels in male P2Y6 ${ }^{\Delta \mathrm{Ncr} 1}$ mice

199 compared to their control littermates (Fig. 1D). Collectively, NK-cell P2Y6R signaling

200 has no major impact on energy homeostasis and body weight control in obesity, while

201 it appears to affect liver weight in obesity.

202

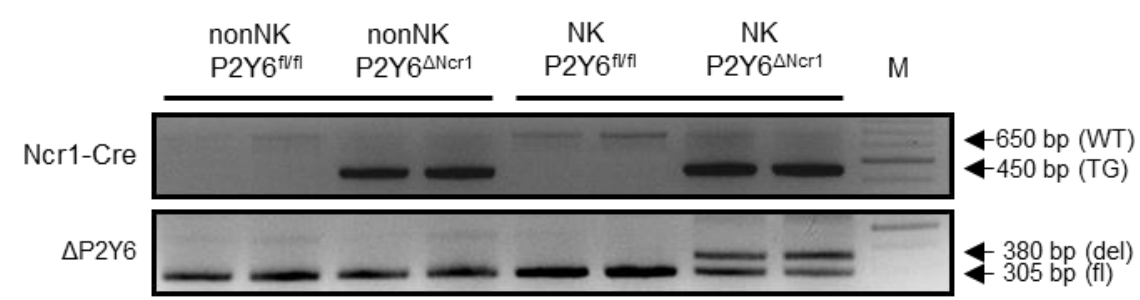

Fig. S2: P2Y6R is selectively deleted in NK cells. Splenic cells from $P 2 Y 6^{f / f i f}(n=2)$ and $P 2 Y 6^{\Delta N c r 1}(n=2)$ mice were separated with magnetic beads resulting in two fractions: NK cells and nonNK cells. Genomic DNA was extracted from each group. Genotyping for P2Y6R was performed by competitive PCR using the primers listed in table 1 which resulted in a 305 bp PCR-product (floxed) and a 370 bp PCR-product (P2Y6R deletion) in NK cells isolated from P2Y6 ${ }^{\Delta \text { Ncr1 }}$ (Ncr1-Cre positive mice (TG band 450 bp)). This deletion band was neither detected in NK cells from P2Y6 ${ }^{\mathrm{fl} / \mathrm{fl}}$, (Ncr1-Cre negative mice (WT band 650

A Body weight development

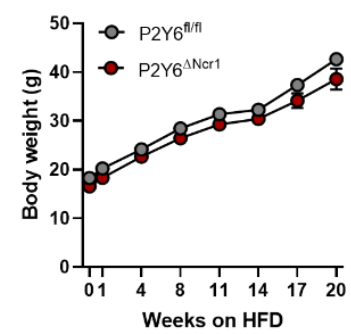

C

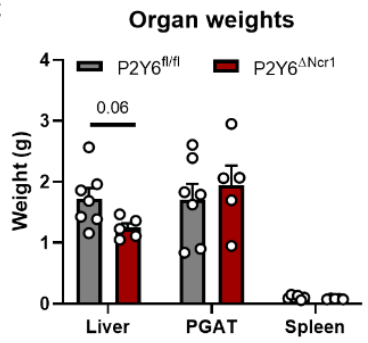

B

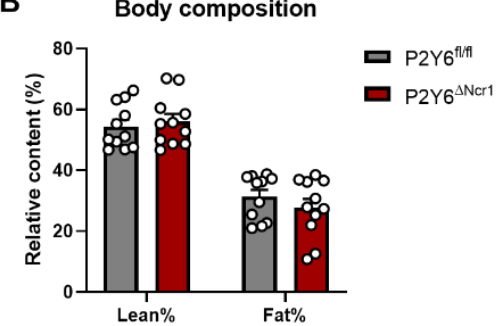

D

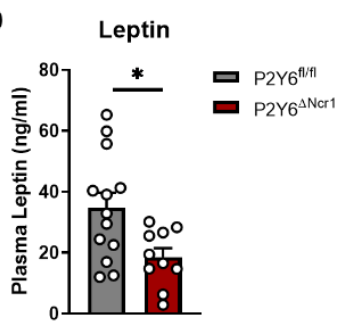

bp)) nor in nonNK cells of either genotype.

Fig. 1: Deletion of the P2Y6R gene from NK cells does not affect energy homeostasis in mice on HFD. (A) Body weight development was assessed over a period of 20 weeks in transgenic mice lacking P2Y6R expression in NK cells (P2Y6 $\left.{ }^{\Delta N c r 1}, n=11\right)$ and littermate control mice $\left(P 2 Y 6^{f / / f l}, n=11\right)$ subjected to HFD feeding. (B) Body composition of P2Y6 ${ }^{f / f 1}(n=11)$ and P2Y6 ${ }^{\Delta N c r 1}(n=11)$ mice after 15-16 weeks on HFD was determined by $\mu-C T$ analysis. (C) Organ weights of P2Y $6^{\mathrm{fl} / \mathrm{fl}}(\mathrm{n}=7)$ and P2Y6 ${ }^{\Delta \mathrm{Ncr} 1}(\mathrm{n}=5)$ mice after 14 weeks on HFD. (D) Plasma leptin levels of P2Y6 $6^{f / f l}(n=13)$ and P2Y6 ${ }^{\Delta N c r 1}(n=10)$ mice fed a HFD for 14 weeks and fasted for 16 hours were analyzed by ELISA. Statistics: (A) Two-way RM ANOVA with Sidak's multiple comparison test. (B-D) Unpaired two-tailed t-test, ${ }^{*} p \leq 0.05$. Data are represented as mean \pm SEM. $\circ$ denotes individual mice. 


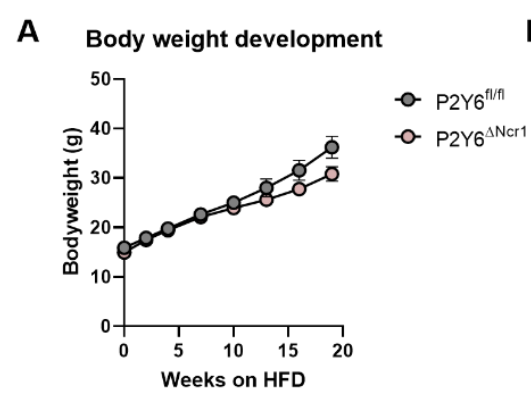

C

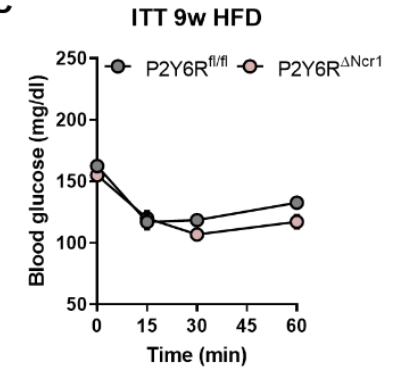

$\mathbf{F}$

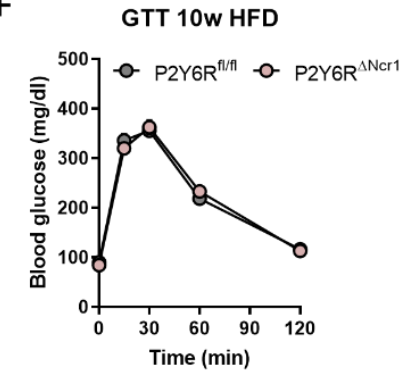

B

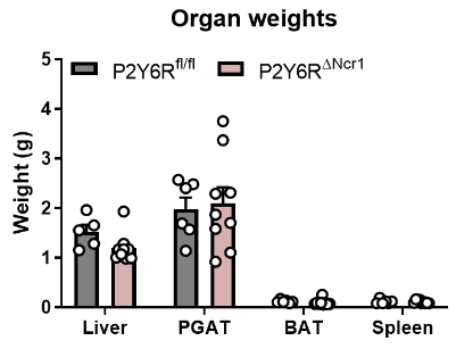

D

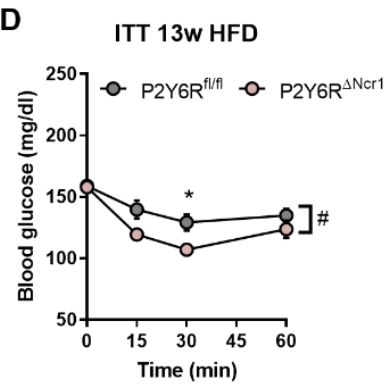

E

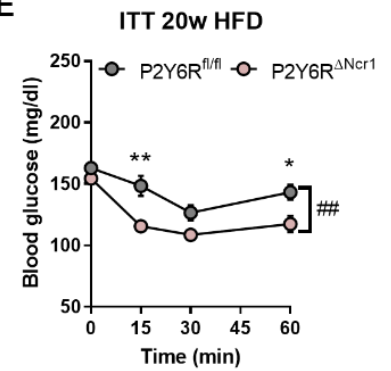

G

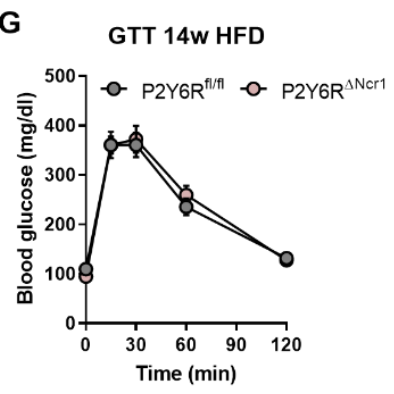

H

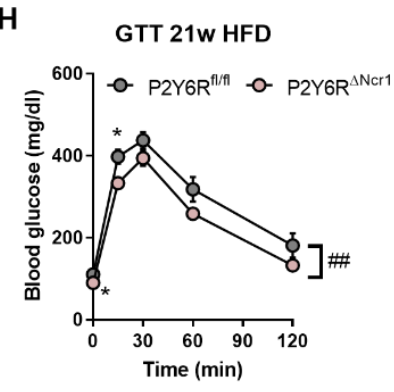

Fig. S3: Deletion of P2Y6R from NK cells improves insulin sensitivity in female mice on HFD. (A) Body weight development in HFD-fed female mice (P2Y6 f/ffl, $\left.n=16 ; P 2 Y 6^{\Delta N c r 1}, n=15\right)$ (B) Organ weights after 16 weeks of HFD-feeding (P2Y6 ${ }^{f / f 1}, n=5$; $\left.P 2 Y 6^{\Delta N c 1}, n=9\right)(C-E)$ Female transgenic mice lacking P2Y6R expression in NK cells (P2Y6 $\left.{ }^{\Delta N c r 1}, n=15\right)$ and littermate control mice $\left(P 2 Y 6^{f / f / l}, n=16\right)$ fed a HFD for 9 weeks (C), 13 weeks (D) and 20 weeks (E) were subjected to an insulin tolerance test (ITT). (F-H) Glucose tolerance tests (GTT) were performed in female P2Y6 $6^{f / f l}(n=16)$ and P2Y6 ${ }^{\Delta N c 1}(n=15)$ after 10 weeks (F), 14 weeks (G) and 21 weeks (H) on HFD. Statistics: (A, C-H) Two-way RM ANOVA with Sidak's multiple comparison test, ${ }^{*} p \leq 0.05,{ }^{* *} p \leq 0.01$; genotype: $\# p \leq 0.05$, \#\#p $\leq 0.01$. Error bars represent SEM. (B, I-K) Unpaired two-tailed t-test, ${ }^{*} p \leq 0.05$. Data are represented as mean \pm SEM. $\circ$ denotes individual mice. 
A

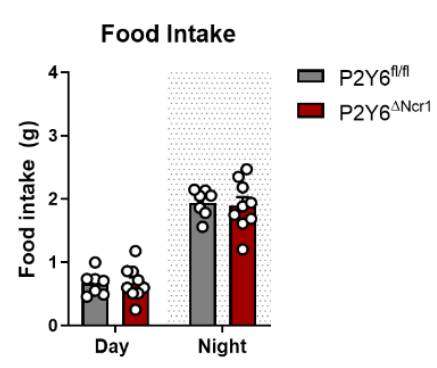

C

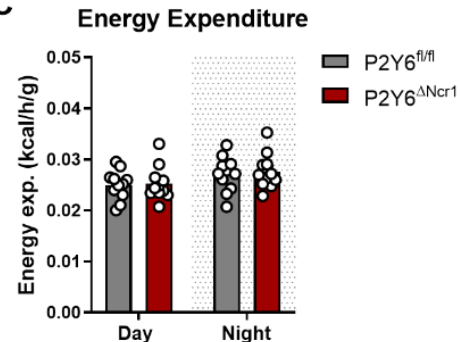

B

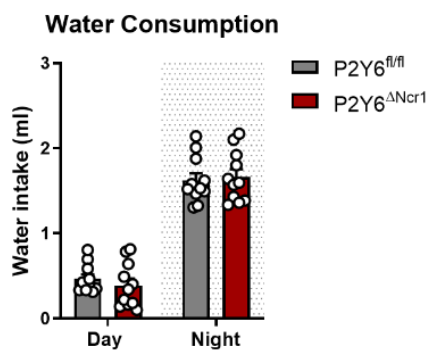

D

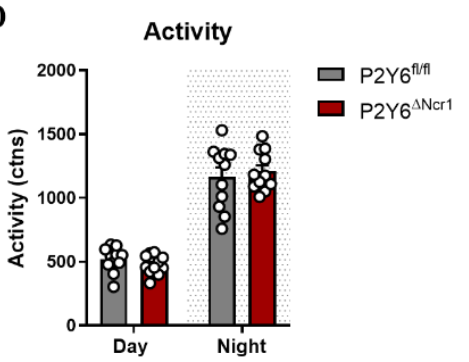

Fig. S4: NK cell-specific deletion of P2Y6R does not affect food intake or energy expenditure in male mice on HFD. Indirect calorimetry was performed in P2YG $6^{f / f l}(n=11)$ and P2Y6 ${ }^{\Delta N c r 1}(n=11)$ mice after 14 weeks on HFD. Data are shown as average values for day and night. (A) Food intake. (B) Water consumption. (C) Energy expenditure normalized to lean mass. (D) Average activity counts. Statistics: (A-D) Unpaired two-tailed t-test, ${ }^{*} p \leq 0.05$. Data are represented as mean \pm SEM. $\circ$ denotes individual mice.

\section{P2Y6R signaling in natural killer cells impairs insulin sensitivity in HFD mice}

207 Next, we aimed to determine the impact of P2Y6R signaling in NK cells on glucose

208 metabolism and insulin sensitivity. To this end, we performed insulin tolerance tests

209 (ITT) and glucose tolerance tests (GTT) at various timepoints upon exposure to CD or

210 HFD. Compared to controls, deletion of the P2Y6R from NK cells improved insulin

211 sensitivity after 13 and 20 weeks of HFD feeding in both genders (Fig. 2A-C and Suppl.

212 Fig. S3C-E) but not in male mice fed a control diet (Suppl. Fig. S5C-E). However,

213 glucose tolerance was not affected by NK cell-specific P2Y6R deletion neither on HFD

214 (Fig. 2D-F and Suppl. Fig. S3F-H) nor on CD (Suppl. Fig. S5F-H). While we did not

215 observe any differences in fasted blood glucose levels between P2Y6 ${ }^{\Delta N c 1}$ mice and

216 their control littermates on HFD (Fig. 2G), fasted insulin concentrations were reduced

217 in P2Y6 ${ }^{\Delta \mathrm{Ncr} 1}$ mice as early as after 10 weeks of HFD feeding (Fig. $2 \mathrm{H}$ ) concomitant with

218 a reduction of the homeostasis model assessment index for insulin resistance (HOMA-

219 IR) (Fig. 2I). These effects were HFD-specific and could not be observed in mice on

220 control diet (Suppl. Fig. S5I-J). Taken together, abrogation of P2Y6R-signaling from NK

221 cells, partially protects from the development of obesity-associated insulin resistance. 

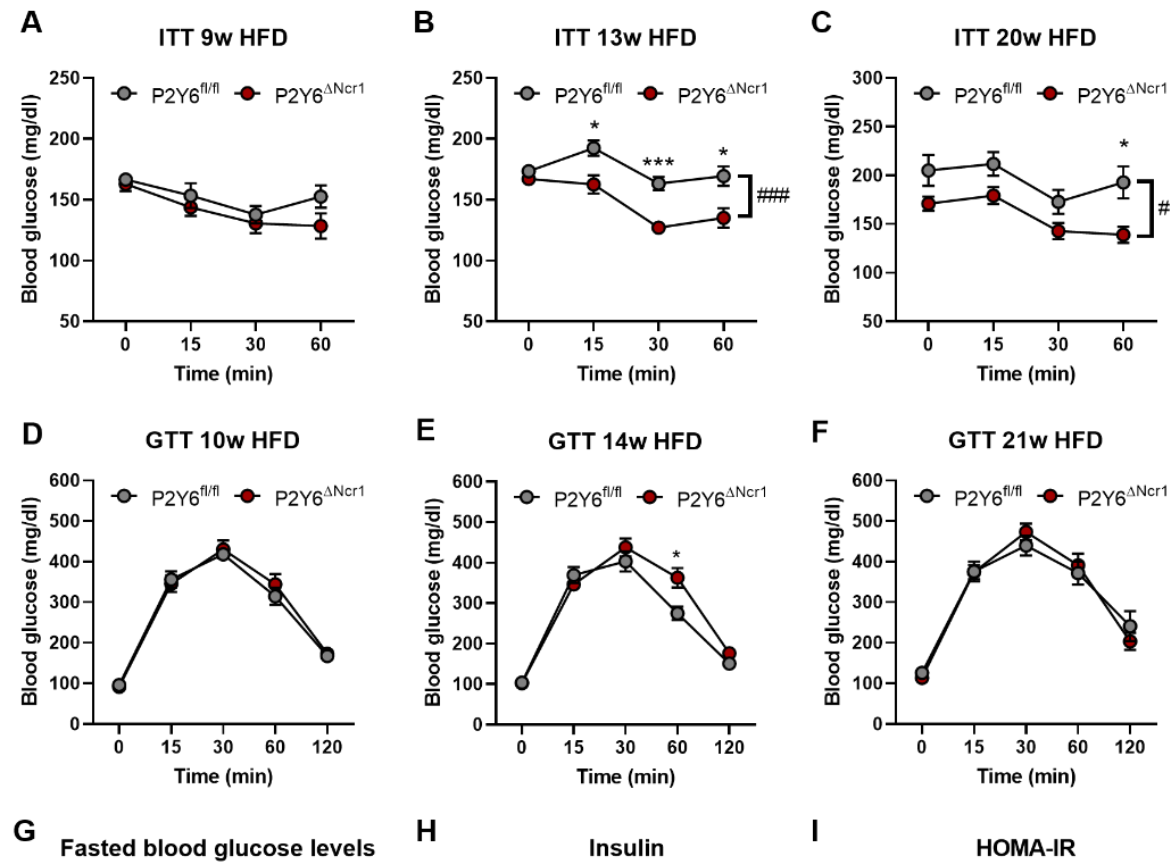

H
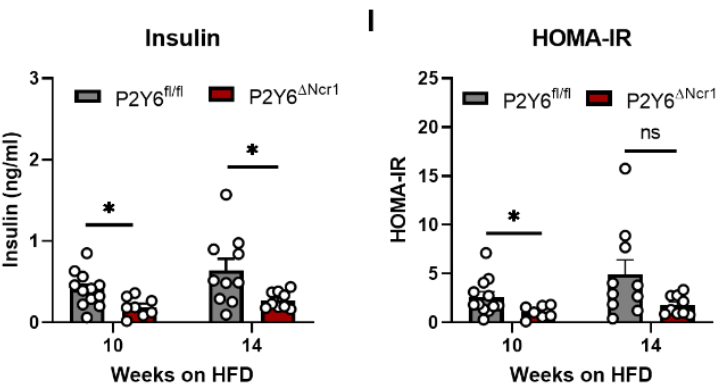

Fig. 2: P2Y6R signaling in natural killer cells impairs insulin sensitivity in HFD-fed mice. (A-C) Transgenic mice lacking P2Y6R expression in NK cells $\left(P 2 Y 6^{\Delta N c r 1}, n=11\right)$ and littermate control mice (P2Y6 $\left.{ }^{f / f l}, n=11\right)$ on HFD for 9 weeks $(A), 13$ weeks $(B)$ and 20 weeks $(C)$ were subjected to an insulin tolerance test (ITT). (D-F) Glucose tolerance tests (GTT) were performed in P2Y6 $6^{\mathrm{fl} / f l}(\mathrm{n}=11)$ and P2Y6 ${ }^{\Delta \text { Ncr1 }}(n=11)$ after 10 weeks (D), 14 weeks (E) and 21 weeks (F) on HFD. (G) Blood glucose levels after 16 hour-fasting in P2Y6 $6^{\text {fl/fl }}(n=11)$ and P2Y6 $6^{\Delta N c r 1}(n=11)$ mice after 10 and 14 weeks on HFD. (H) Plasma insulin levels of P2Y6 ${ }^{\text {fl/fl }}(n=10-12)$ and P2Y6 ${ }^{\Delta N c r 1}(n=8-9)$ mice fed a HFD for 14 weeks and fasted for 16 hours were analyzed by ELISA. (I) Homeostasis Model Assessment (HOMA) Index for insulin resistance $(I R)$ was determined for $P 2 Y 6^{f l / f l}(n=10-12)$ and $P 2 Y 6^{\Delta N c r 1}(n=7-9)$ mice after 10 and 14 weeks on HFD. Statistics: (A-F) Two-way RM ANOVA with Sidak's multiple comparison test, * $p \leq 0.05$, ${ }^{* *} p \leq 0.01,{ }^{* * *} p \leq 0.001$; genotype: \#p $\leq 0.05, \# \#$, $\leq 0.001$. Error bars represent SEM. (G-I) Unpaired twotailed t-test, ${ }^{*} p \leq 0.05$. Data are represented as mean \pm SEM. o denotes individual mice. 
A Body weight development

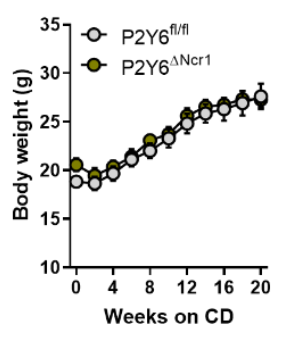

C

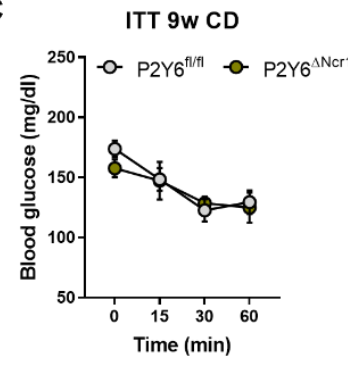

$\mathbf{F}$

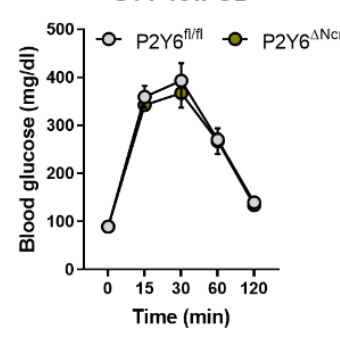

I

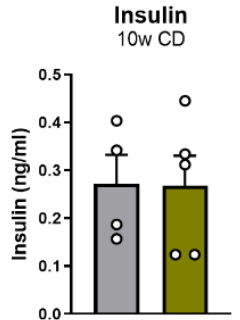

B
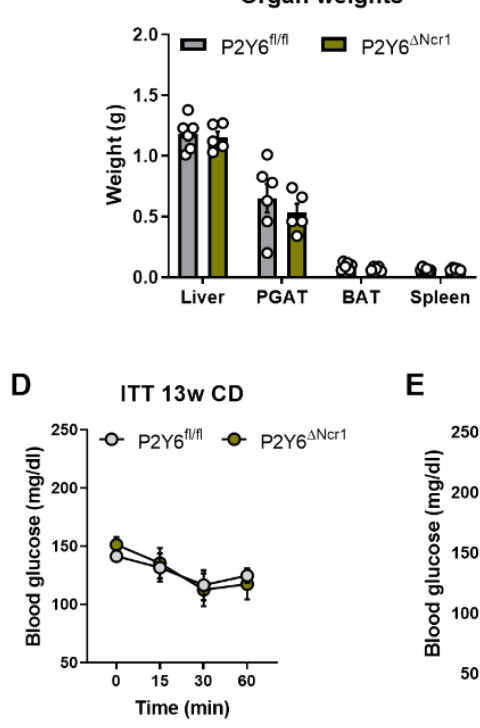

E

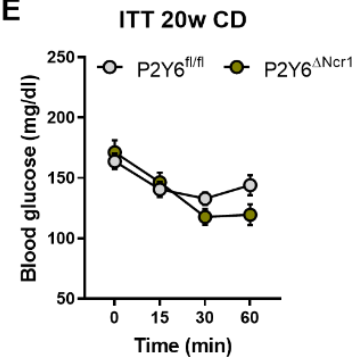

G

GTT 14w CD

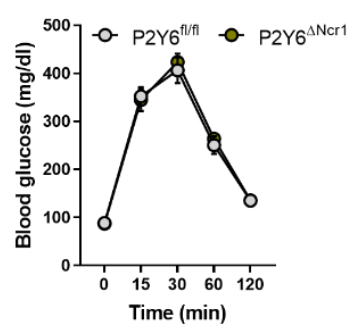

H

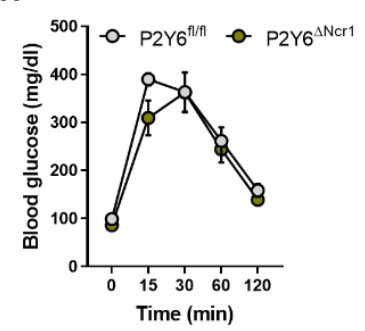

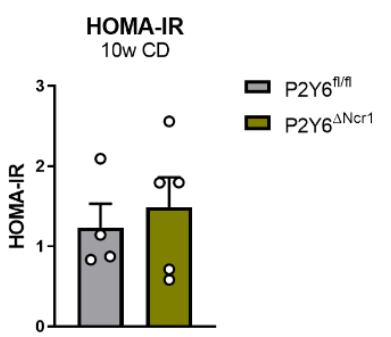

Fig. S5: P2Y6R deletion in NK cells of control diet-fed mice does not improve insulin sensitivity. (A) Body weight development in control diet-fed male mice (P2Y6 $\left.6^{f / f l}, n=6 ; P 2 Y 6^{\Delta N c r 1}, n=5\right)$. (B) Organ weights after 21 weeks of $C D$-feeding (P2Y6 ${ }^{f / f l}, n=6$; $\left.P 2 Y 6^{\Delta N c r 1}, n=5\right)$. (C-E) Transgenic mice lacking P2Y6R expression in NK cells (P2Y6 $\left.{ }^{\Delta N c r 1}, n=5\right)$ and littermate control mice $\left(P 2 Y 6^{f / f / l}, n=6\right)$ fed a $C D$ for 9 weeks (C), 13 weeks (D) and 20 weeks (E) were subjected to an insulin tolerance test (ITT). (F-H) Glucose tolerance tests (GTT) were performed in P2Y6 $6^{f / f l}(n=6)$ and P2Y6 ${ }^{\Delta N c r 1}(n=5)$ after 10 weeks $(F)$, 14 weeks (G) and 21 weeks (H) on CD. (I) Plasma insulin levels of P2YG $6^{f / f l}(n=4)$ and P2Y6 ${ }^{\Delta N c r 1}(n=6)$ mice fed a CD for 10 weeks and fasted for 16 hours were analyzed by ELISA. (J) Homeostasis Model Assessment (HOMA) Index for insulin resistance (IR) was determined for P2Y6 $6^{f / f l}(n=4)$ and P2Y6 ${ }^{\Delta N c r 1}$ $(n=6)$ mice after 10 weeks on CD. Statistics: $(A, C-H)$ Two-way RM ANOVA with Sidak's multiple comparison test. (B, I-J) Unpaired two-tailed t-test. Data are represented as mean \pm SEM. $\circ$ denotes individual mice. 
226 NK cell-specific deletion of P2Y6R improves insulin-induced suppression of

227 hepatic glucose production in obesity

228 To further dissect tissue-specific effects of P2Y6R signaling in NK cells on insulin

229 sensitivity and glucose homeostasis, we performed hyperinsulinemic-euglycemic

230 clamp studies in HFD-fed P2Y6 ${ }^{\Delta N c r 1}$ mice and their littermate controls. Mice with NK

231 cell-specific depletion of the P2Y6R required significantly higher glucose infusion rates

232 (GIR) compared to their littermate controls (Fig. 3A) in order to maintain euglycemic

233 blood glucose levels during the steady state of the clamp experiment (Fig. 3B), while

234 exhibiting comparable levels of circulating human insulin concentrations (Fig. 3C).

235 Quantitative assessment of insulin's ability to suppress gluconeogenesis in both

236 groups of mice revealed that P2Y6 $6^{\Delta \mathrm{Ncr} 1}$ mice exhibited a clear improvement in insulin's

237 ability to suppress hepatic glucose production (HGP) (Fig. 3D). Since hepatic glucose

238 production is controlled both through insulin-induced changes in the expression of key

239 enzymes of gluconeogenesis as well as through gluconeogenic substrate availability,

240 such as fatty acids released from adipose tissue through lipolysis, we next compared

241 the ability of insulin to regulate the concentration of plasma free fatty acid (FFA)

242 concentrations during the clamp phase. This analysis revealed that while in obese

243 HFD-fed control mice insulin failed to suppress circulating plasma FFA concentrations

244 during the clamp, insulin significantly reduced circulating FFA concentrations in

245 P2Y6 ${ }^{\Delta \mathrm{Ncr} 1}$ mice (Fig. 3E).

246 Lipolysis is exceptionally sensitive to the action of insulin (Jensen and Nielsen,

247 2007) and tightly regulated by the phosphorylation of hormone sensitive lipase (HSL)

248 in adipose tissue (Nielsen et al., 2014). We found that insulin inhibits the

249 phosphorylation of $\mathrm{HSL}$ at serine 563 in $\mathrm{P} 2 \mathrm{Y}^{\Delta \mathrm{Ncr} 1}$ mice (Fig. 3F), which is a main

250 phosphorylation site known to regulate HSL-driven lipolysis (Nielsen et al., 2014), while

251 phosphorylation of serine 565 only showed a mild increase in P2Y6 ${ }^{\Delta \mathrm{Ncr} 1}$ mice (Fig. 3F).

252 However, in contrast to an enhanced ability of insulin to suppress HGP and

253 adipose tissue lipolysis, insulin-stimulated uptake of $\left[{ }^{14} \mathrm{C}\right]-\mathrm{D}$-glucose into perigonodal

254 adipose tissue (PGAT), brown adipose tissue (BAT), brain, and skeletal muscle (SKM)

255 remained unaltered in P2Y6 ${ }^{\Delta N c r 1}$ mice compared to their controls (Fig. 3G). Consistent

256 with unaltered insulin-stimulated glucose uptake in these organs, also assessment of

257 insulin-stimulated protein kinase B (AKT) phosphorylation during the clamp failed to

258 reveal significant differences in $\mathrm{P} 2 \mathrm{Y}^{\Delta \mathrm{Ncr} 1}$ mice compared to their controls (Fig. 4).

259 Moreover, insulin's ability to activate AKT phosphorylation in liver did not differ between 
bioRxiv preprint doi: https//doi.org/10.1101/2021.06.23.449596; this version posted June 24, 2021. The copyright holder for this preprint (which was not certified by peer review) is the author/funder, who has granted bioRxiv a license to display the preprint in perpetuity. It is made available under aCC-BY 4.0 International license.

260 the two groups of animals (Fig. 4). Thus, deletion of P2Y6R from NK-cells in obesity

261 appears to enhance insulin-stimulated suppression of lipolysis in PGAT and thus

262 gluconeogenic substrate supply to liver.

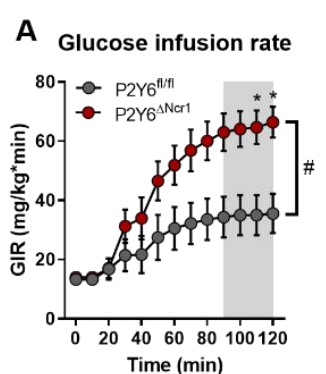

E

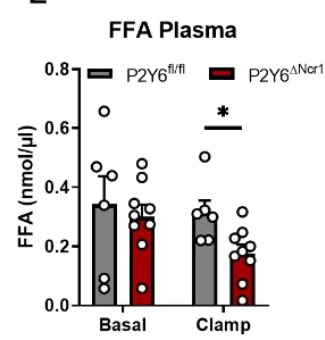

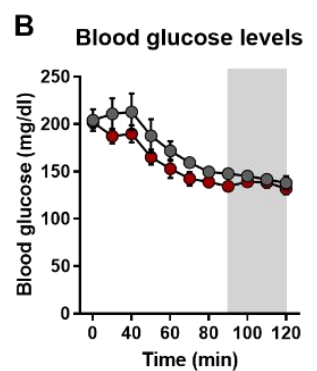

$\mathbf{F}$

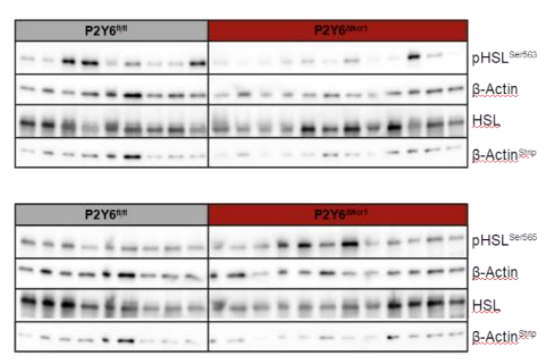

Hepatic
glucose production

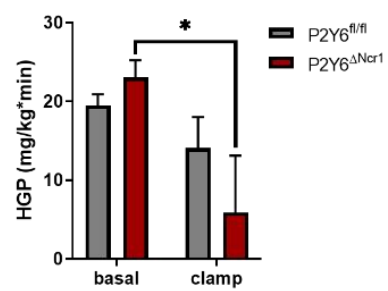

HSL phosphorylation

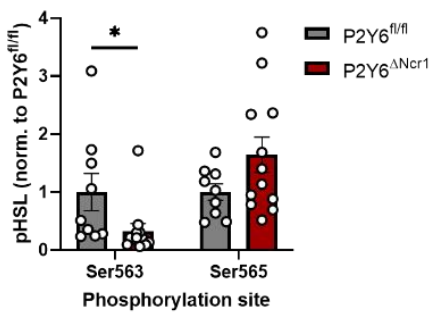

G

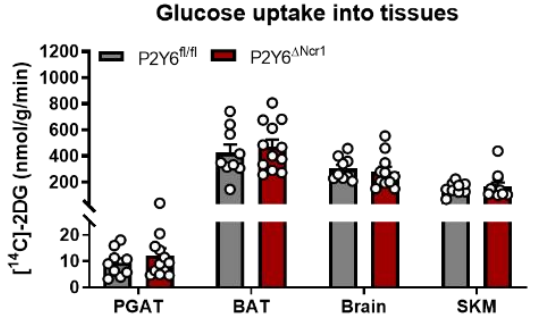

Fig. 3: P2Y6R deletion in NK cells reduces hepatic glucose production and lipolysis in adipose tissue. Transgenic mice lacking P2Y6R expression in NK cells (P2Y6 $6^{\Delta N r 1}, n=12$ ) and littermate control mice (P2Y6 fl/fl, $n=9$ ) were subjected to HFD feeding for 9 weeks. (A and B) Hyperinsulinemic-euglycemic clamp study (A) Glucose-infusion rate (GIR) required to reach euglycemic steady state glucose levels (90-120 min). (B) Blood glucose levels, monitored every $10 \mathrm{~min}$ during the experiment, reached euglycemic steady state levels at the end of the experiment. (C) Human insulin levels in plasma during the clamped state were determined by ELISA (P2Y6 fl/fl,$\left.n=9 ; P 2 Y 6^{\Delta N c r 1}, n=12\right)$. (D) Hepatic glucose production (HGP) $\left(P 2 Y 6^{f / / f l}, n=8 ; P 2 Y 6^{\Delta N c r 1}, n=9\right)$ and $(E)$ free fatty acid (FFA) concentration in plasma $\left(P 2 Y 6^{f / f l}, n=6 ; P 2 Y 6^{\Delta N c r 1}, n=9\right)$ were determined at the basal and clamped state. (F) Organs of clamped $\mathrm{P}^{2} 6^{\mathrm{fl} / \mathrm{fl}}(\mathrm{n}=9)$ and P2Y6 ${ }^{\Delta \mathrm{Ncr}}(\mathrm{n}=12)$ mice on HFD (9 weeks) were further investigated by western blot analysis. Representative immuno-blots for phosphorylated hormone-sensitive lipase (HSL) at Ser563 and at Ser565, total HSL and $\beta$-Actin in PGAT of clamped mice. Quantification of pHSLSer563 and pHSL Ser565 levels was performed after normalization to $\beta$-Actin and total HSL levels. (G) Organ-specific glucose uptake rates during the clamped state in P2Y6 ${ }^{f l / f l}(n=9)$ and P2Y6 $6^{\Delta N c_{1}}(n=12)$ mice. Statistics: (A) Two-way RM ANOVA with Sidak's multiple comparison test. *p $\leq 0.05$, \#p (Genotype) $\leq 0.05$. (B) Two-way RM ANOVA with Sidak's multiple comparison test for steady state $(90-120 \mathrm{~min}) \mathrm{p}=0.2598$. (CH) Unpaired two-tailed t-test, * $p \leq .0 .05$. Data are represented as mean \pm SEM. o denotes individual mice. 

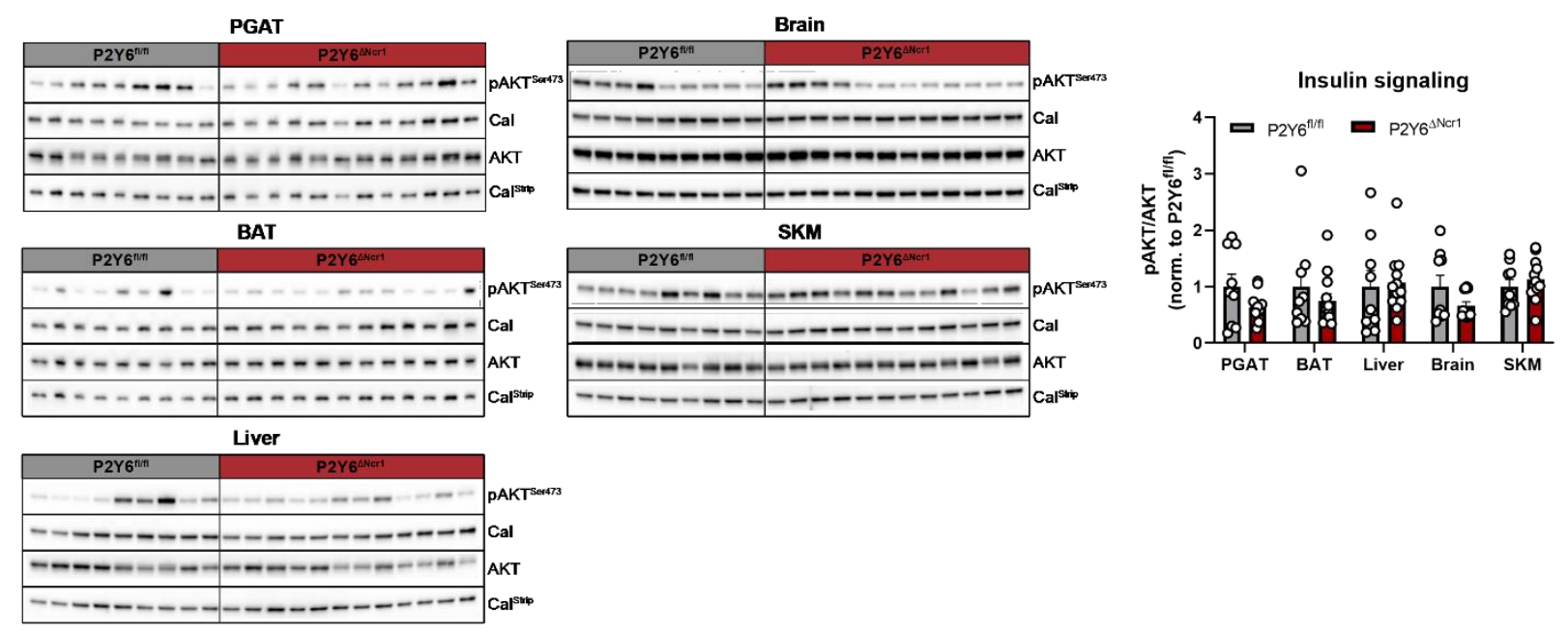

Fig. 4: NK cell-specific deletion of P2Y6R does not alter insulin signaling in peripheral organs. Organs of clamped P2Y6 $6^{f / / 1}(n=9)$ and P2Y6 $6^{\Delta N c r 1}(n=12)$ mice on HFD (9 weeks) were further investigated by western blot analysis. Representative immuno-blots for phosphorylated AKT (Ser473), total AKT and Calnexin in PGAT, BAT, liver, brain and SKM of clamped mice. Quantification of pAKT Ser473 levels was performed after normalization to Calnexin and total AKT as loading controls. Statistics: Unpaired twotailed t-test, ${ }^{*} p \leq 0.05$. Data are represented as mean \pm SEM. $\circ$ denotes individual mice.

264

265 NK cell-specific deletion of P2Y6R upregulates genes associated with 266 triglyceride homeostasis and increases mitochondrial function in liver

267 To further investigate the effects of NK cell-specific P2Y6R signaling on liver and 268 adipose tissue, we compared gene expression profiles of liver and PGAT from $269 \mathrm{P} 2 \mathrm{Y}^{\Delta \mathrm{Ncr} 1}$ and their control littermates on HFD by mRNA deep sequencing. Differential 270 gene expression analysis (DESeq2) identified 166 differentially expressed genes in 271 liver and 58 genes in PGAT of P2Y6 ${ }^{\Delta N c r 1}$ mice compared to their controls (Fig. 5A). To 272 gain more functional insights based on the gene expression patterns, we subjected 273 significantly regulated genes (cutoff $p \leq 0.05$ ) to gene set enrichment analyses. The 274 three most significantly regulated gene ontologies in liver identified pathways of 275 acylglycerol homeostasis, triglyceride homeostasis and oxidative phosphorylation (Fig. 276 5B), gene set enrichment analysis for regulated genes in PGAT did not yield any 277 metabolism-related gene ontology terms (data not shown). Here, expression of

278 lipoprotein lipase $(L p /)$, a marker gene of the GO terms for acylglycerol and triglyceride

279 homeostasis, was shown to be specifically up-regulated in liver of P2Y6 ${ }^{\Delta N c r 1}$ mice 280 compared to controls, but not in PGAT (Fig 5C). In contrast, Plin2 expression was 281 significantly down-regulated in PGAT and showed a mild decrease in expression in 282 liver (Fig. 5D). 
Interestingly, analysis of the overlap of commonly regulated genes in liver and

284 PGAT revealed that 10 genes (Lilra5, Rora, Herc1, Bnip2, Lactb, Ciao2a, Rps27I, Ice2,

285 Anxa2, Vps13c) were similarly differentially expressed in both organs (Suppl. Fig. S6).

286 Importantly, some of them are known to be specifically expressed in immune cells and

287 NK cells, thus raising the possibility that some of the commonly observed differences

288 in both tissues might result from their coordinated expression changes in tissue-

289 infiltrating NK cells. Here, only Lilra5 expression was found to be up-regulated,

290 whereas the expression of Rora and Herc1 was down-regulated (Suppl. Fig. S6).

291 These findings were further validated by real-time PCR analysis. Consistent with the

292 results obtained in the mRNA sequencing experiment, Rora expression was

293 significantly down-regulated in liver and PGAT of P2Y6 ${ }^{\Delta N c r 1}$ mice compared to the

294 expression in littermate controls (Fig. 5E) and Lilra5 expression was confirmed to be

295 up-regulated in both organs of P2Y6R knock out mice (Fig. 5F).

296 As triglyceride (TG) homeostasis was affected by NK cell-specific deletion of

297 P2Y6R, we conducted lipidomic analyses of liver samples from mice fed a HFD for 9

298 weeks. These analyses revealed no significant overall changes in hepatic TG (Fig. 5G)

299 and diacylglyceride (DAG) contents (Fig. 5H), however some specific lipid species

300 showed a trend towards reduction in liver of P2Y6 ${ }^{\Delta \mathrm{Ncr} 1}$ compared to controls (C16:0,

301 C18:0 TGs, C16:0-C18:1, C18:1-C18:1 DAGs).

302 Since hepatic lipid homeostasis is closely linked to mitochondrial function in liver,

303 we next investigated the rates of oxidative capacity and extracellular acidification rates

304 (ECAR) in micro-punches from liver of HFD-fed P2Y6 ${ }^{\Delta N c r 1}$ mice and their control

305 littermates. This analysis revealed a significant upregulation of the ECAR (Fig. 5I),

306 indicating an increase in glycolysis, while the oxygen consumption rate (OCR) was

307 only slightly but not significantly improved in liver of HFD-fed P2Y6 ${ }^{\Delta \mathrm{Ncr} 1}$ mice compared

308 to their control littermates (Fig. 5I).

309 Collectively, NK cell-specific P2Y6R signaling appears to affect triglyceride

310 metabolism and mitochondrial function in liver, while coordinately regulated differences

311 in gene expression between liver and adipose tissue possibly arise from NK cell

312 intrinsic changes in gene expression in tissue-infiltrating NK cells. 
bioRxiv preprint doi: https://doi.org/10.1101/2021.06.23.449596: this version posted June 24,2021 . The copyright holder for this preprint (which was not certified by peer review) is the author/funder, who has granted bioRxiv a license to display the preprint in perpetuity. It is made available under aCC-BY 4.0 International license.

A

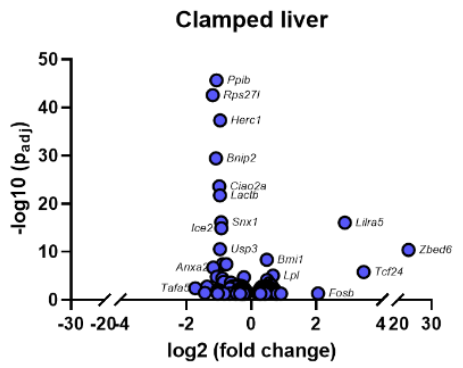

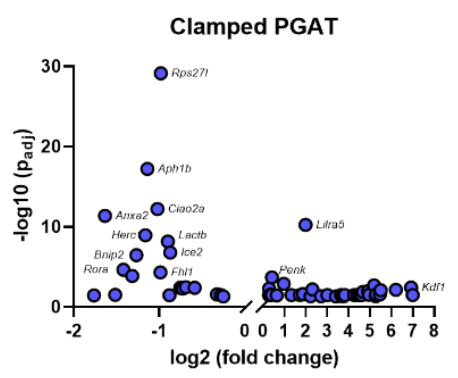

B
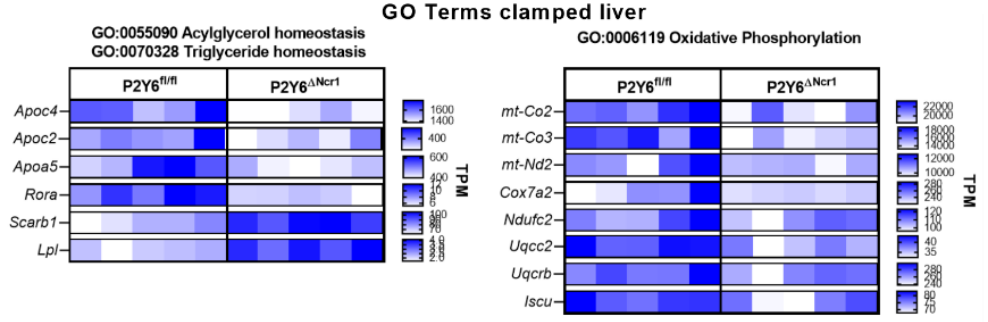

C

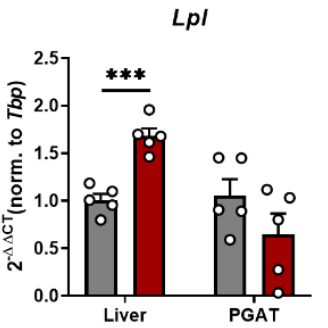

D

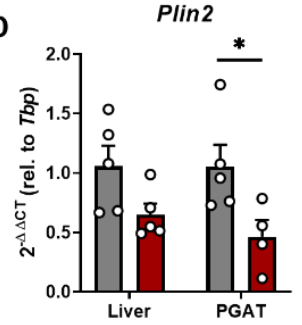

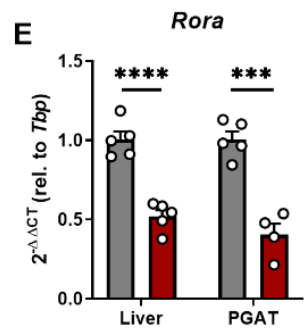

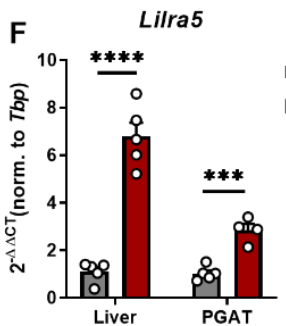

G

Hepatic Triacylglycerides

\section{H}

Hepatic Diacylglycerides

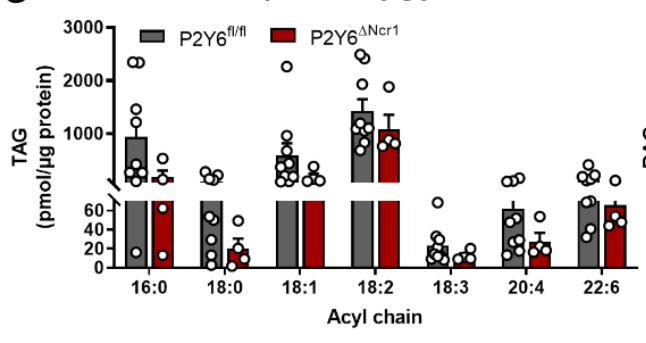

OCR/ECAR basal

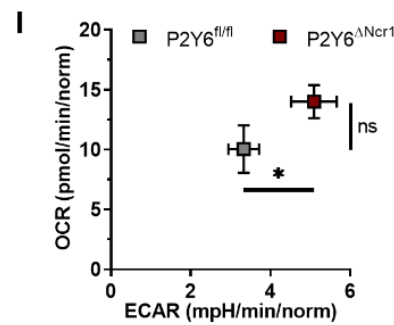

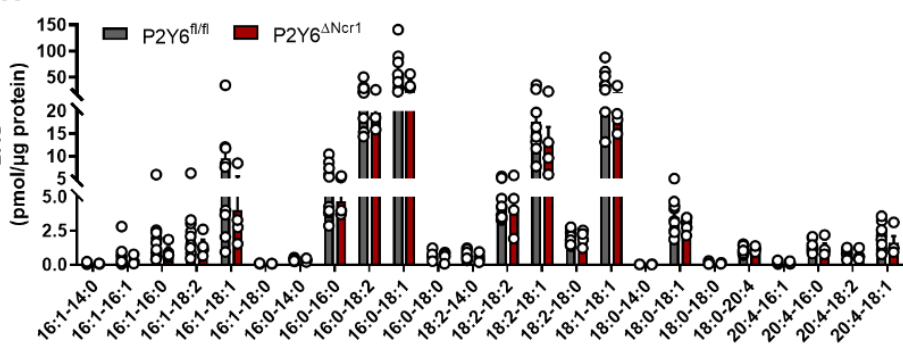

Acyl chain

Fig. 5: NK cell-specific deletion of P2Y6R upregulates genes associated with triglyceride homeostasis and increases mitochondrial function in liver. (A) Differentially expressed genes in liver (left) and PGAT (right) of clamped P2Y6 $6^{f / f l}(n=5)$ versus P2Y6 ${ }^{\Delta N c r 1}(n=5)$ mice on HFD (9 weeks). (B) Gene set enrichment analysis of transcriptomes in livers of clamped P2Y6 $6^{f / f l}(n=5)$ versus P2Y $6^{\Delta N c r 1}$ $(n=5)$ mice. Shown are the most significantly enriched gene ontology terms (GO terms) with the transcripts per million (TPM) for the GO term defining marker genes. (C-F) Validation of the RNASeq data by real-time PCR analysis. Expression of lipoprotein lipase $(L p l)$, RAR-related orphan receptor alpha (Rora), Perilipin 2 (Plin2) and Leukocyte immunoglobulin-like receptor subfamily A member 5 (Lilra5) was quantified in clamped liver and PGAT of P2Y6 $6^{f / f l}(n=5)$ and P2Y6 $\Delta$ Nor1 $(n=5)$ mice. Quantification was determined by the $2^{-\Delta \Delta C t}$ method using the housekeeping gene TATA-binding protein 
(Tbp) for normalization. (G-H) Lipidomic analysis of livers from P2Y6 $6^{f / f l}(n=8)$ and P2Y6 $6^{\Delta N c r 1}(n=4)$ mice on HFD (9 weeks). (I) Seahorse analysis in liver punches of P2Y6 $6^{f / f l}(n=5)$ and P2Y6 ${ }^{\Delta N c r 1}(n=4)$ mice on HFD (9 weeks). Baseline oxygen consumption rate (OCR) as measure for respiration was plotted against the extracellular acidification rate (ECAR) as measure for glycolysis. Statistics: (C-I) Unpaired two-tailed t-test, ${ }^{*} p \leq 0.05,{ }^{* *} p \leq 0.01,{ }^{* * *} p \leq 0.001,{ }^{* * * *} p \leq 0.0001$. Data are represented as mean \pm SEM. $\circ$ denotes individual mice.
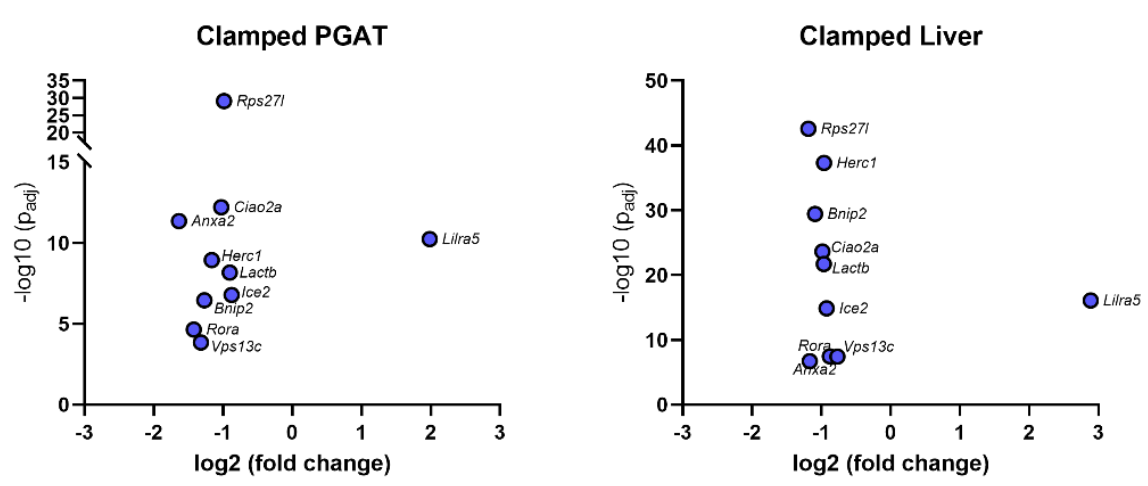

Fig. S6: Overlap analysis of differentially expressed genes in clamped PGAT and liver reveal a NK cell-specific gene set. Differentially expressed genes $(p \leq 0.05)$ in clamped PGAT and liver were compared and overlapping genes plotted as volcano plots.

317 NK cell specific deletion of P2Y6R alters tissue-infiltration of immune cells and 318 expression of chemokines

319 NK cells represent a heterogeneous innate lymphocyte cell population with organ- and 320 microenvironment-specific expression of cytotoxic molecules, receptors and 321 transcription factors (Crinier et al., 2018; Zhao et al., 2020). As the overlap analysis of 322 the transcriptional changes from adipose tissue and liver revealed a common set of 323 regulated, possibly immune cell-specific genes, we next assessed the transcriptome 324 of isolated NK cells. To this end, NK cells were isolated from adipose tissue and liver 325 of HFD-fed P2Y6 ${ }^{\Delta \mathrm{Ncr} 1}$ and P2Y6 $6^{\text {fl/fl }}$ mice by flow cytometry cell sorting based on the 326 expression of the NK cell-specific surface markers NK1.1 and Ncr1 (Suppl. Fig. S7).

327 DESeq2 analysis of the transcriptome of bulk-sequenced NK cells resulted in 59 328 differentially expressed genes in adipose tissue-derived NK cells (Fig. 6A) and 78 329 genes in hepatic NK cells (Fig. 6B). Gene set enrichment analyses of the differentially 330 expressed genes (cutoff $\mathrm{p} \leq 0.05$ ) in adipose tissue-derived NK cells identified pathways 331 of lymphocyte chemotaxis, lymphocyte migration and neutrophil migration (Fig. 6C), 
332 whereas pathways of protein folding, and chaperone cofactor-dependent protein

333 folding were detected in liver-derived NK cells (Fig. 6D).
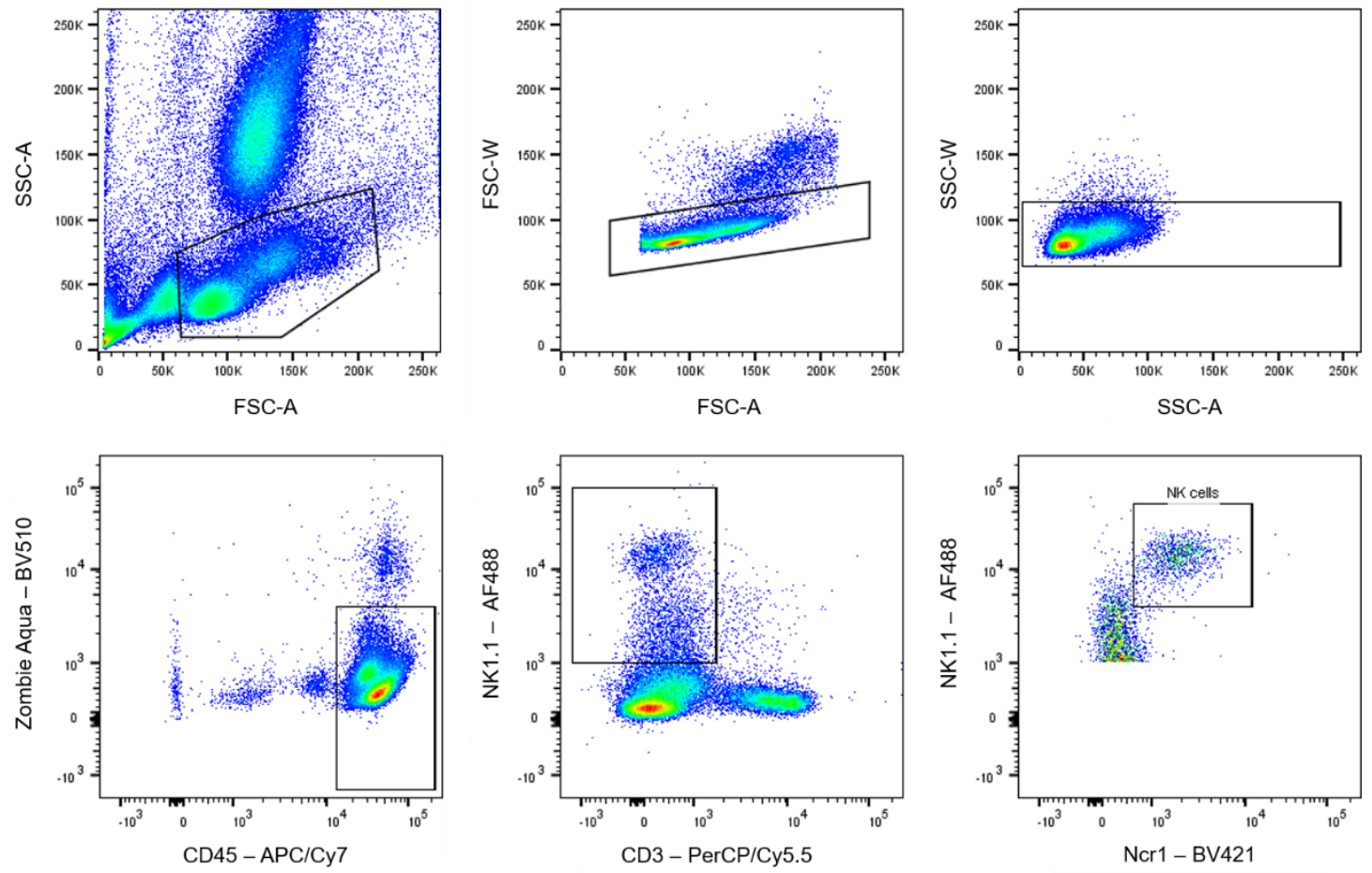

Fig. S7: NK cells were sorted from liver, PGAT and spleen of male mice on HFD by flow cytometry. NK cells were gated as single/viable/CD45 $/$ CD3-/NK1.1+/Ncr1 ${ }^{+}$cells.

Consistent with our previous findings in liver and adipose tissue, we also found

336 genes of the presumably NK cell-specific gene set to be differentially expressed in 337 isolated NK cells of both tissue origins (Fig. 6E). This supports our hypothesis that 338 similar differences in gene expression between liver and adipose tissue possibly arise

339 from NK cell intrinsic changes in gene expression of tissue-infiltrating NK cells. Besides

340 this first NK cell-specific gene set, we found a second set of overlapping genes in 341 isolated NK cells derived from adipose tissue and liver. This gene set includes the up342 regulated genes Trip4, Stip1, Gtf2a2 and Hsph1 and the down-regulated genes Rab8b, 343 Usp3, Ppib, F2rl3 and Snx1 (Fig. 6F). Usp3, Ppib and Snx1 have also been found to 344 be differentially expressed in liver and Rab8b in adipose tissue of clamped P2Y6 ${ }^{\Delta \mathrm{Ncr} 1}$ 345 mice when compared to their littermate controls (data not shown). Notably, in adipose tissue-derived NK cells the gene expression of the 347 chemokines $\mathrm{Ccl}$, Ccl4, Xcl1 and $\mathrm{Cxcl16}$ (Fig. 6G) was found to be down-regulated in 348 parallel with the down-regulation of Csf1 expression in hepatic NK cells (Fig. 6B). As 349 these chemokines are involved in the recruitment and activation of immune cells, we 350 aimed to elucidate the impact of this P2Y6R-dependent and NK cell-specific down- 
351 regulation on the infiltration of immune cells into different organs. In line with this

352 reduced expression of chemokines in NK cells, we observed a significant reduction of

353 NK cell numbers in liver and spleen, a decrease in NKT cell numbers in PGAT and a

354 reduction of macrophages in spleen of P2Y6 $6^{\Delta \mathrm{Ncr} 1}$ mice compared to control (Fig. 7A).

355 In addition, infiltration of MAC2 positive macrophages into adipose tissue was reduced

356 in mice with NK cell-specific P2Y6R deletion after prolonged HFD-feeding (Suppl. Fig.

357 S8A), concomitant with a mild but non-significant reduction in the expression of 358 inflammatory cytokines and chemokines (Suppl. Fig. S8B). On the contrary, expression

359 of inflammatory markers in liver of mice fed a HFD for 22 weeks were not significantly

360 altered between genotypes (Suppl. Fig. S8C).

361 Depending on their maturation state, NK cells express a distinct repertoire of 362 cytotoxins and cytokines (Crinier et al., 2018). As we observed a reduction in 363 chemokine expression in P2Y6 ${ }^{\Delta N c r 1}$-derived NK cells, we investigated the impact of 364 P2Y6R signaling on the maturation state of NK cells based on the surface expression 365 of CD27 and CD11b. Quantification of the different maturation subsets of NK cells by 366 flow cytometry analysis revealed that the majority of NK cells in adipose tissue and 367 liver belong to the mature subset (CD11 $\left.{ }^{+} / \mathrm{CD}^{-} 7^{-}\right)$, whereas in spleen and blood also 368 a notable proportion of immature $\left(\mathrm{CD} 11 \mathrm{~b} / \mathrm{CD} 27^{+}\right)$and intermediate $\left(\mathrm{CD} 11 \mathrm{~b}^{+} / \mathrm{CD} 27^{+}\right)$ 369 NK cells could be found (Fig. 7B). However, abrogation of P2Y6R signaling did not 370 affect the overall maturation state in NK cells (Fig. 7B).

371 In summary, P2Y6R signaling in NK-cells of HFD-fed mice likely increases the 372 expression of chemokines favoring the infiltration of immune cells into metabolically 373 relevant organs, while it at the same time drives NK cell intrinsic changes in the 374 expression of specific gene sets that might serve as possible markers for tissue375 infiltrating NK cells. 
bioRxiv preprint doi: https://doi.org/10.1101/2021.06.23.449596: this version posted June 24, 2021. The copyright holder for this preprint (which was not certified by peer review) is the author/funder, who has granted bioRxiv a license to display the preprint in perpetuity. It is made available under aCC-BY 4.0 International license.

A

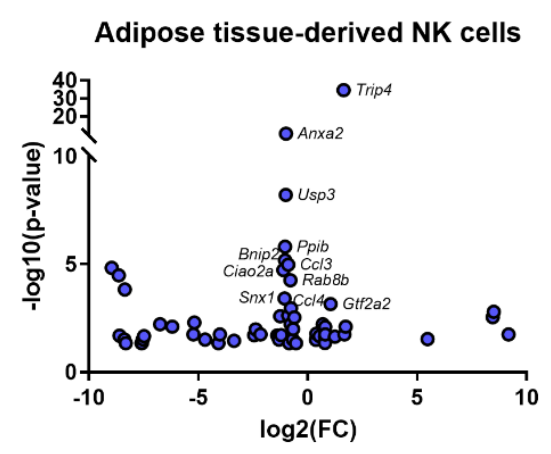

B

Liver-derived NK cells

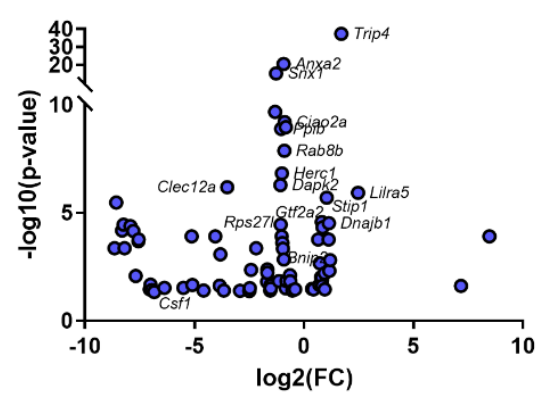

E

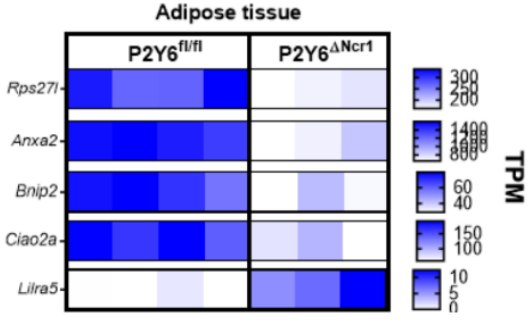

$\mathbf{F}$
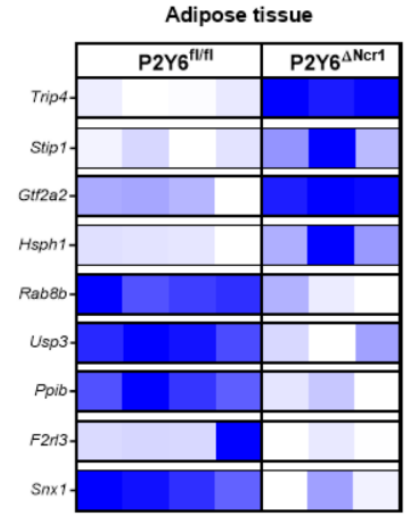

G

GO:0048247 Lymphocyte chemotaxis

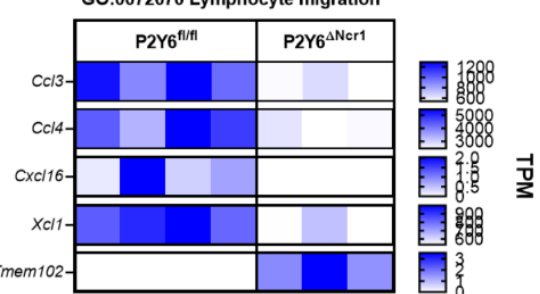

GO Terms adipose tissue-derived NK cells Top 25 sig. terms, ordered by overlap

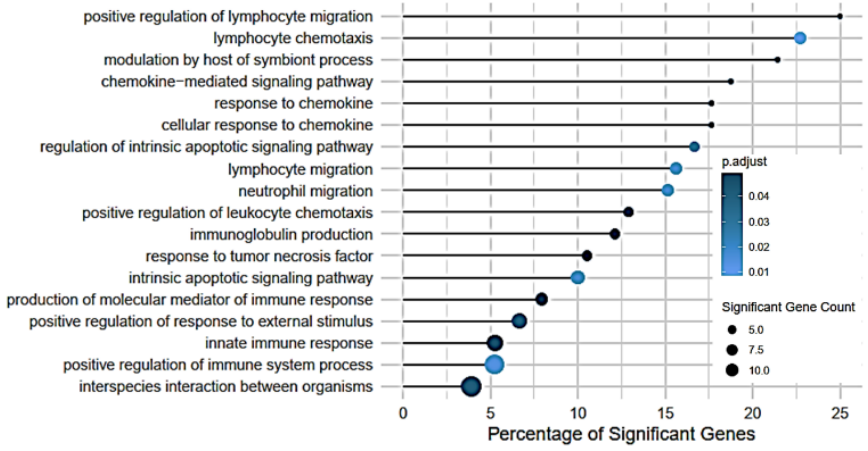

D

GO Terms liver-derived NK cells Top 25 sig. terms, ordered by overlap
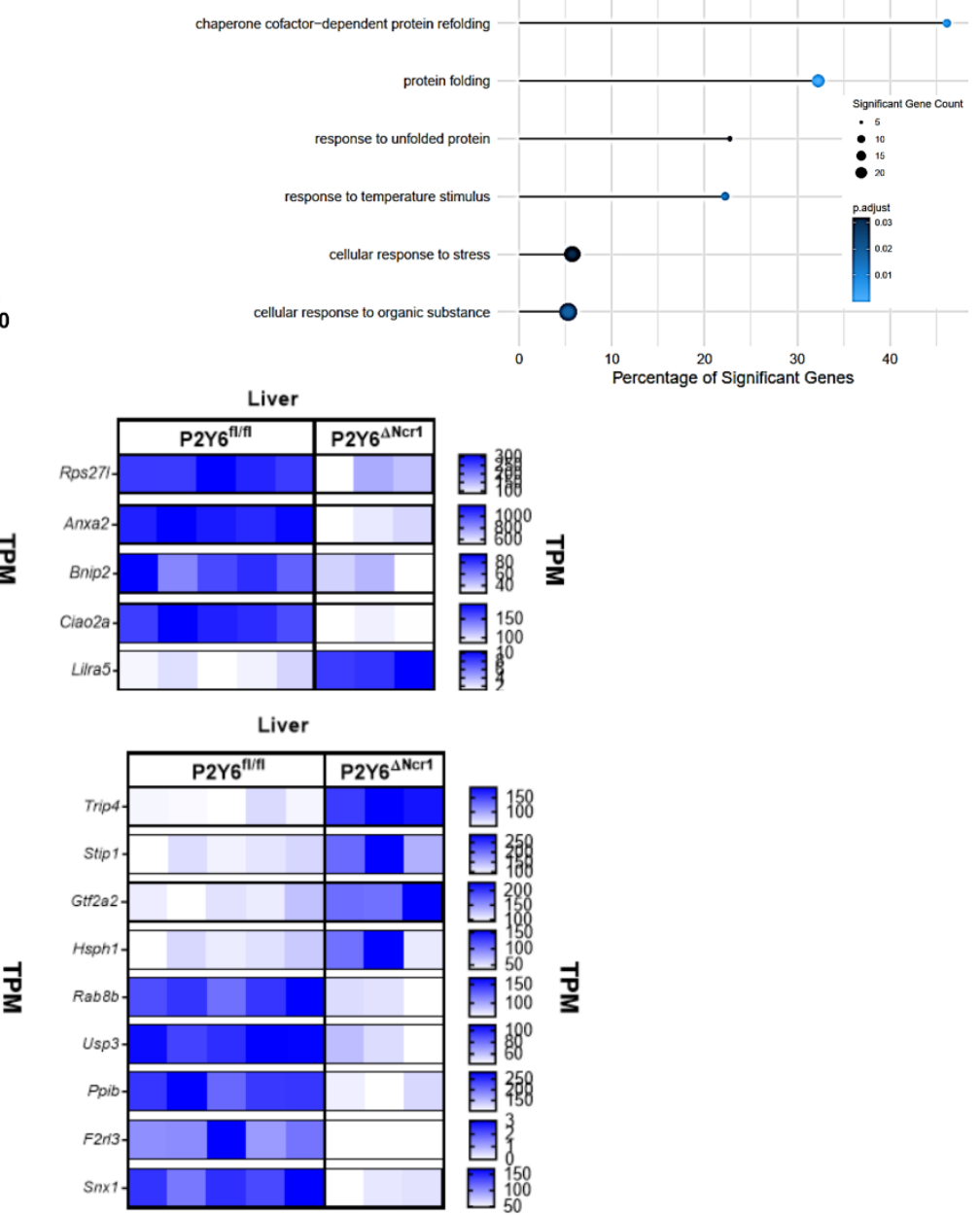

Fig. 6: Adipose tissue-derived NK cells from P2Y6 ${ }^{\Delta \mathrm{Ncr} 1}$ mice show reduced expression of chemokines. (A-B) Transcriptomic analysis of NK cells (defined as single/viable/CD45 $/$ CD3$/ \mathrm{NK} 1.1^{+} / \mathrm{Ncr}^{+}{ }^{+}$cells and sorted by flow cytometry) from adipose tissue $(\mathrm{A})$ and liver $(\mathrm{B})$ of $\mathrm{P} 2 \mathrm{Y} 6^{\mathrm{fl} / \mathrm{fl}}(\mathrm{n}=4-$ 
bioRxiv preprint doi: https://doi.org/10.1101/2021.06.23.449596: this version posted June 24,2021 . The copyright holder for this preprint (which was not certified by peer review) is the author/funder, who has granted bioRxiv a license to display the preprint in perpetuity. It is made available under aCC-BY 4.0 International license.

5) and P2Y6 ${ }^{\Delta N c r 1}(n=3)$ mice on HFD (15 weeks). Volcano-plots of differentially expressed genes with $p \leq 0.05$. (C-D) $G O$ term analysis of differentially expressed genes with $p \leq 0.05$ in adipose tissue-derived NK cells (C) and (D) hepatic NK cells. (E) TPM plots of commonly differentially expressed genes in NK cells isolated from PGAT and from liver. These genes have also been found to be differentially expressed in liver and PGAT of clamped mice. (F) TPM plots of genes differentially expressed in NK cells from both origins, PGAT (left) and liver (right). (G) TPM plots of GO term defining genes differentially expressed in adipose tissue-derived NK cells of $P 2 \mathrm{Y}^{\mathrm{ft} / \mathrm{fl}}(\mathrm{n}=4)$ and $\mathrm{P} 2 \mathrm{YG}^{\Delta \mathrm{Ncr} 1}(\mathrm{n}=3)$ mice.

A
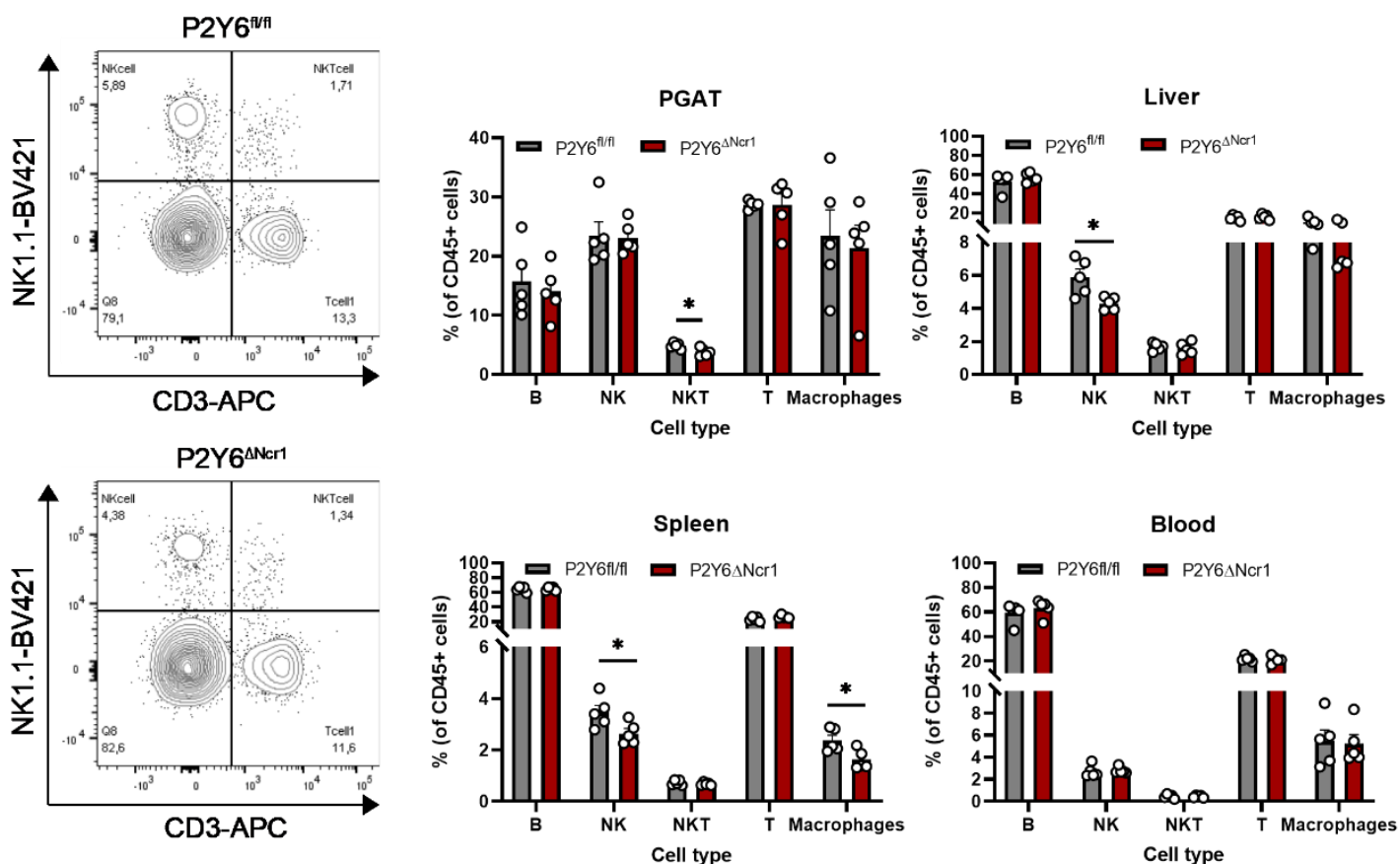

B
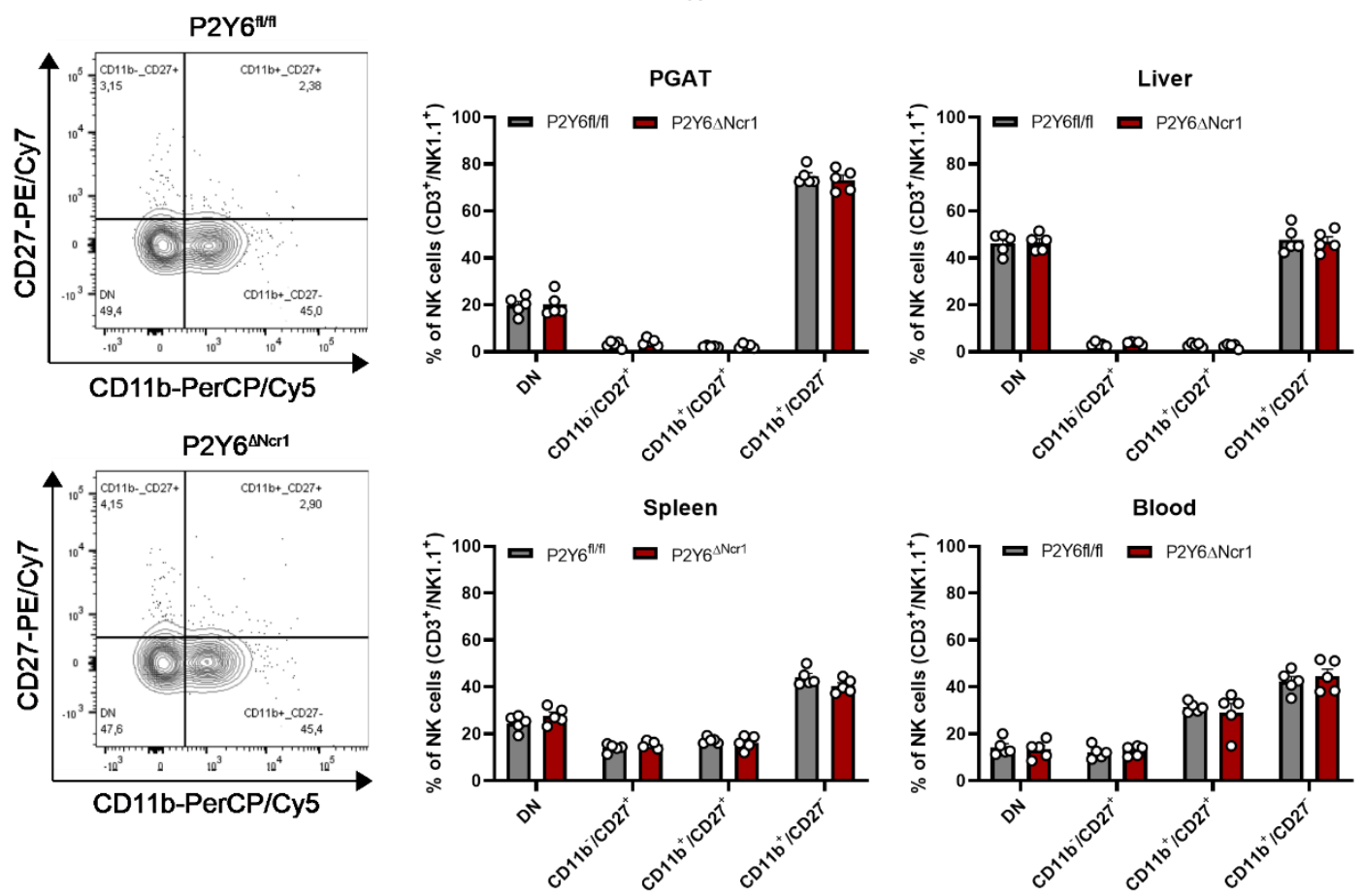

Fig. 7: P2Y6 ${ }^{\Delta N c r 1}$ mice exhibit reduced immune cell infiltration into peripheral organs. (A) Representative flow cytometry analysis plots and quantification of immune cell subsets in PGAT, liver, spleen and blood of P2Y6 $6^{\text {fl/fl }}(n=5)$ and P2Y6 ${ }^{\Delta N c r 1}(n=5)$ mice fed a HFD for 14 weeks. B cells were 
gated as single/viable/CD45 $/ \mathrm{CD} 3 / \mathrm{CD} 19^{+}$cells, NK cells as single/viable/CD45 $/ \mathrm{CD} 3 / \mathrm{NK} 1.1^{+}$cells, NKT cells as single/viable/CD45 $/ \mathrm{CD} 3^{+} / \mathrm{NK} 1.1^{+}$cells, $\mathrm{T}$ cells as single/viable/CD45 $/ \mathrm{CD} 3^{+} / \mathrm{NK} 1.1^{-}$cells and macrophages as single/viable/CD $45^{+} / \mathrm{CD} 11 \mathrm{~b}^{+} / \mathrm{F} 4 / 80^{+}$cells. (B) Representative FACS plots and quantification of the maturation status of NK cells in PGAT, liver, spleen and blood of P2Y6 $6^{\mathrm{fl} / \mathrm{fl}}(\mathrm{n}=5)$ and P2Y6 ${ }^{\Delta \mathrm{Ncr} 1}(\mathrm{n}=5)$ mice fed a HFD for 14 weeks. NK cells were gated as single/viable/CD45 $/$ CD $3 /$ NK1 $11^{+}$ cells and further subdivided into double negative (DN) (CD11b-CD27-), immature (CD11b-/CD27 ${ }^{+}$, intermediate $\left(\mathrm{CD} 11 \mathrm{~b}^{+} / \mathrm{CD} 27^{+}\right)$and mature $\left(\mathrm{CD} 11 \mathrm{~b}^{+} / \mathrm{CD} 27^{-}\right)$NK cells. Statistics: (A, B) Unpaired twotailed t-test, ${ }^{*} p \leq 0.05$. Data are represented as mean \pm SEM. $\circ$ denotes individual mice.

A
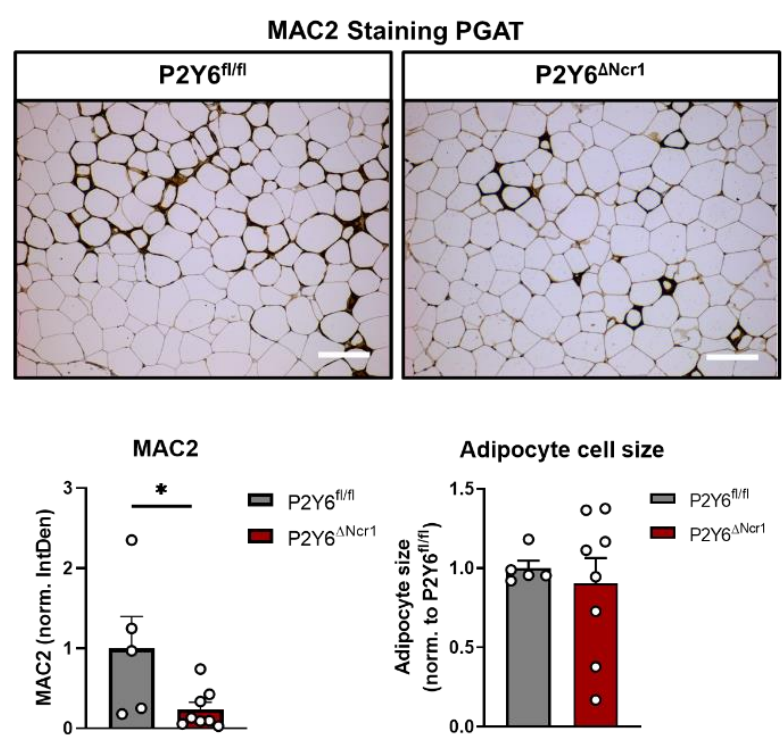

B

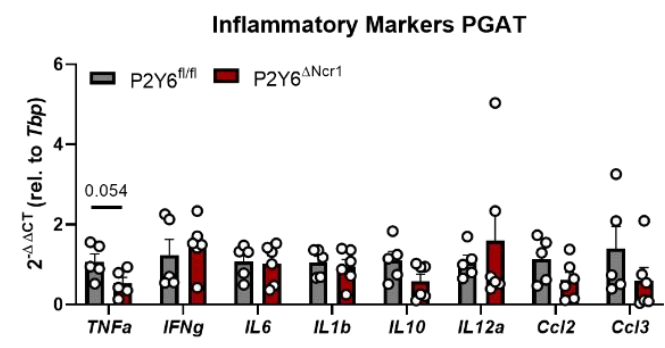

C

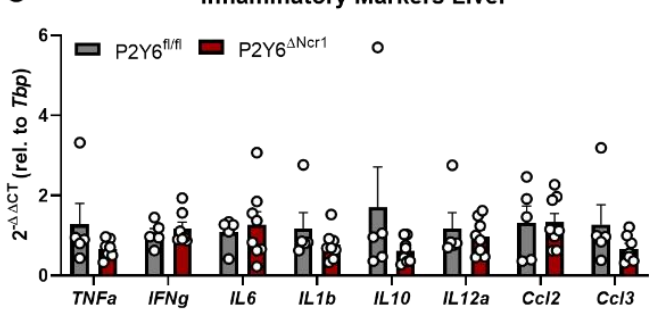

Fig. S8: NK cell-specific deletion of $P 2 Y 6 R$ reduces macrophage infiltration into adipose tissue in mice on HFD. Transgenic mice $\left(P 2 Y 6^{\Delta N c r 1}, n=8\right)$ and their control littermates $\left(P 2 Y 6^{f / f / 1}, n=5\right)$ were fed a HFD for 22 weeks. (A) Immunohistochemical analysis of MAC2 positive macrophages in PGAT of $P 2 Y^{\Delta N c r 1}(n=8)$ and P2Y6 ${ }^{f / / f}$ mice $(n=5)$. Scale bars $100 \mu \mathrm{m}$. (B-C) Real-time PCR analysis of PGAT (B) and liver (C) from $P 2 Y^{\Delta N c r 1}(n=6-8)$ and their control littermates $\left(P 2 Y 6^{f / f / l}, n=5\right)$. Statistics: Unpaired twotailed t-test, ${ }^{*} p \leq 0.05$. Data are represented as mean \pm SEM. $\circ$ denotes individual mice.

380 Abrogation of P2Y6R-signaling in macrophages does not protect from HFD381 induced insulin resistance

382 Since P2Y6Rs are also expressed in macrophages (Bar et al., 2008; Lattin et al., 2008)

383 where its' activation increases the expression of inflammatory (Bar et al., 2008) or 384 regulatory cytokines (Oishi et al., 2016), we also investigated the role of P2Y6R 385 signaling in macrophages in the context of obesity and the development of 386 metaflammation-induced insulin resistance. Therefore, we crossed mice carrying a 387 loxP-flanked P2Y6R gene (Jain et al., 2020) with LysM-Cre mice (Clausen et al., 1999). 388 Gene expression analysis of cultured bone marrow-derived macrophages 389 (BMDM) isolated from control (P2Y6 $\left.{ }^{f / / f l}\right)$ or macrophage-specific P2Y6R-deleted 390 (P2Y6 $\left.{ }^{\Delta L y s M}\right)$ mice confirmed successful and specific reduction of P2Y6R expression in 
391 macrophages (Suppl. Fig. S9A). As obesity development is accompanied by a

392 macrophage switch from M2- to M1-polarized macrophages in adipose tissue (Castoldi

393 et al., 2016), we aimed to elucidate the role of macrophage polarization on P2Y6R

394 expression. Here, we found a more than three-fold upregulation of P2Y6R expression

395 in M2-polarized compared to M1-polarized bone marrow-derived macrophages (Suppl.

396 Fig. S9B).

397 We then investigated the impact of macrophage-specific P2Y6R signaling on

398 the metabolic phenotype of HFD-fed mice. Mice with macrophage-specific deletion of

399 P2Y6R showed the same body weight development as their control littermates (Fig.

$4008 \mathrm{~A}$ ) as well as no differences in body fat content (Fig. 8B). In parallel, investigations of

401 insulin sensitivity and glucose tolerance after 10, 14 or 20 weeks of HFD-feeding did

402 not reveal any significant differences between P2Y6 ${ }^{\Delta L y s M}$ and P2Y6 $6^{f / f l}$ mice (Fig. 8C-H).

403 This was in line with results from indirect calorimetry in mice fed a HFD for 18 weeks.

404 Analyses of food intake, water consumption, energy expenditure, total activity or

405 respiratory exchange rate (RER) did not reveal any differences between $P 2 Y 6^{\Delta L y s M}$ and

406 P2Y6 fl/fl mice (Suppl. Fig. S9C-G). Also, insulin and leptin levels in plasma of 16 hours-

407 fasted mice did not differ between genotypes concomitant with unaltered HOMA-IR 408 (Fig. 8I-K).

409 Since UDP levels are increased in adipocytes (Suppl. Fig. S1H) where it can

410 serve as a "find me" signal triggering macrophages to eliminate necrotic cells, such as

411 hypertrophic and necrotic adipocytes (Cinti et al., 2005), we examined the infiltration

412 of macrophages into adipose tissue of HFD-fed mice by immunohistochemistry.

413 However, we did not find any differences in the amount of MAC2 positive macrophages

414 between in P2Y6 ${ }^{\Delta \text { LysM }}$ and P2Y6 f/fl mice (Suppl. Fig. S10). In line with the overall

415 inconspicuous phenotype of P2Y6 $6^{\Delta \mathrm{LysM}}$ mice, we also did not observe any differences

416 in lipid accumulation in liver between genotypes (Suppl. Fig. S10).

417 In conclusion, we demonstrated here that P2Y6R-signaling in macrophages

418 does not play a major role in the context of obesity-induced insulin resistance and

419 metaflammation. 
A

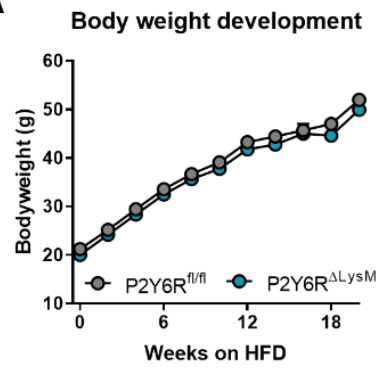

C

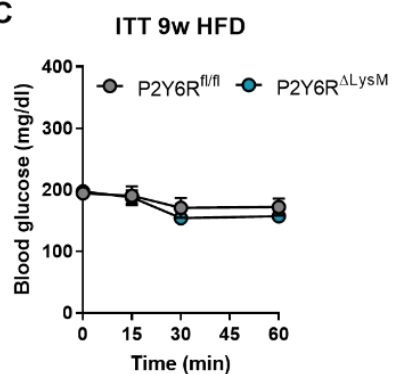

$\mathbf{F}$

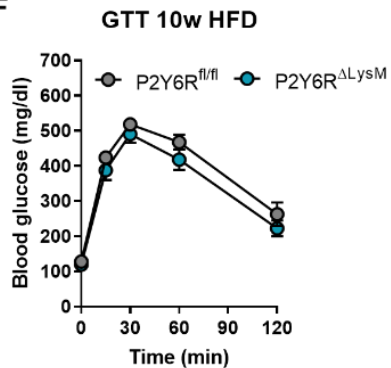

I

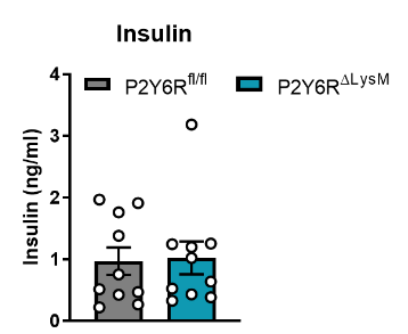

B

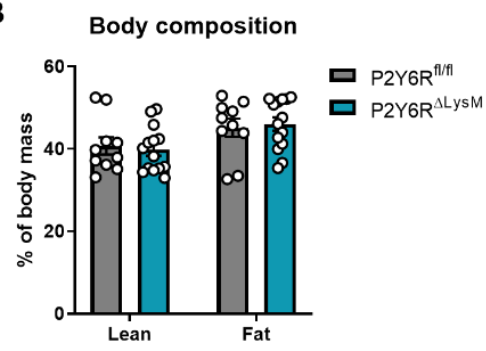

D

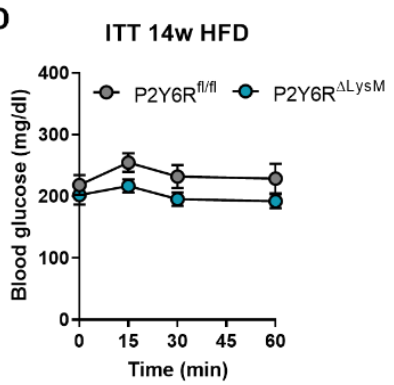

E

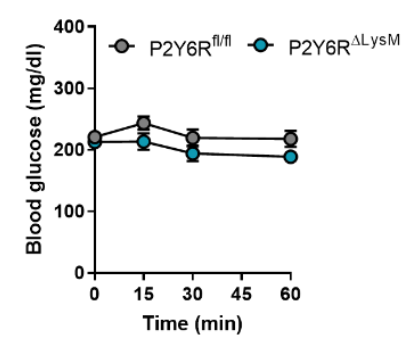

G

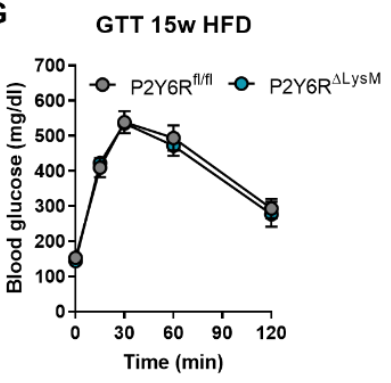

H

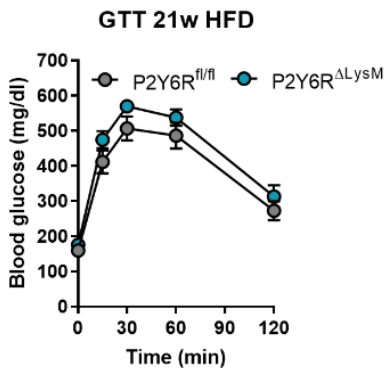

$\mathbf{J}$

Leptin

K

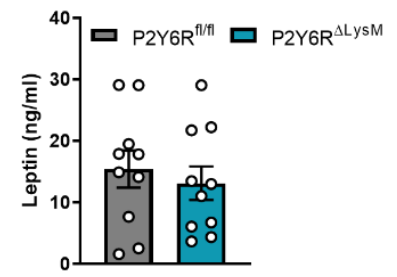

HOMA-IR

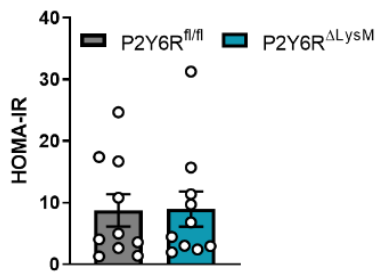

Fig. 8: Deletion of P2Y6R in macrophages does not affect glucose metabolism in mice on HFD. (A) Body weight development was assessed over a period of 20 weeks in transgenic mice lacking P2Y6R expression in macrophages $\left(P 26^{\Delta L y s M}, n=14\right)$ and littermate control mice $\left(P 2 Y 6^{f / / f l}, n=10\right)$ subjected to high-fat diet (HFD) feeding. (B) Body composition was investigated by $\mu C T$ measurements after 19 weeks on HFD (P2Y6 fl/fl, $\left.n=10 ; P 2 Y 6^{\Delta L y s M}, n=14\right)(C-E)$ Transgenic mice lacking P2Y6R expression in macrophages (P2Y6 $\left.{ }^{\Delta L y s M}, n=14\right)$ and littermate control mice $\left(P 2 Y 6^{f / f f l}, n=10\right)$ fed a HFD for 9 weeks (C), 13 weeks (D) and 20 weeks (E) were subjected to an insulin tolerance test (ITT). (F-H) Glucose tolerance tests (GTT) were performed in P2Y $f^{f / f l}(n=10)$ and P2YG ${ }^{\Delta L y s M}(n=14)$ after 10 weeks (F), 14 weeks (G) and 21 weeks (H) on HFD. (I) Plasma insulin levels of P2YG ${ }^{f / / f l}(n=10)$ and P2Y6 $6^{\Delta L y s M}$ $(n=10)$ mice fed a HFD for 10 weeks and fasted for 16 hours were analyzed by ELISA. (J) Blood leptin levels after 16 hour-fasting in P2Y6 $6^{f / f l}(n=10)$ and P2Y6 ${ }^{\Delta L y s M}(n=10)$ mice after 10 weeks on HFD. (K) Homeostasis Model Assessment (HOMA) Index for insulin resistance (IR) was determined for P2Y6 f//fl $(n=10)$ and P2Y6 ${ }^{\Delta L y s M}(n=10)$ mice after 10 weeks on HFD. Statistics: $(A, C-H)$ Two-way RM ANOVA with Sidak's multiple comparison test. (B, I-K) Unpaired two-tailed t-test, ${ }^{*} p \leq 0.05$. Data are represented as mean \pm SEM. $\circ$ denotes individual mice. 

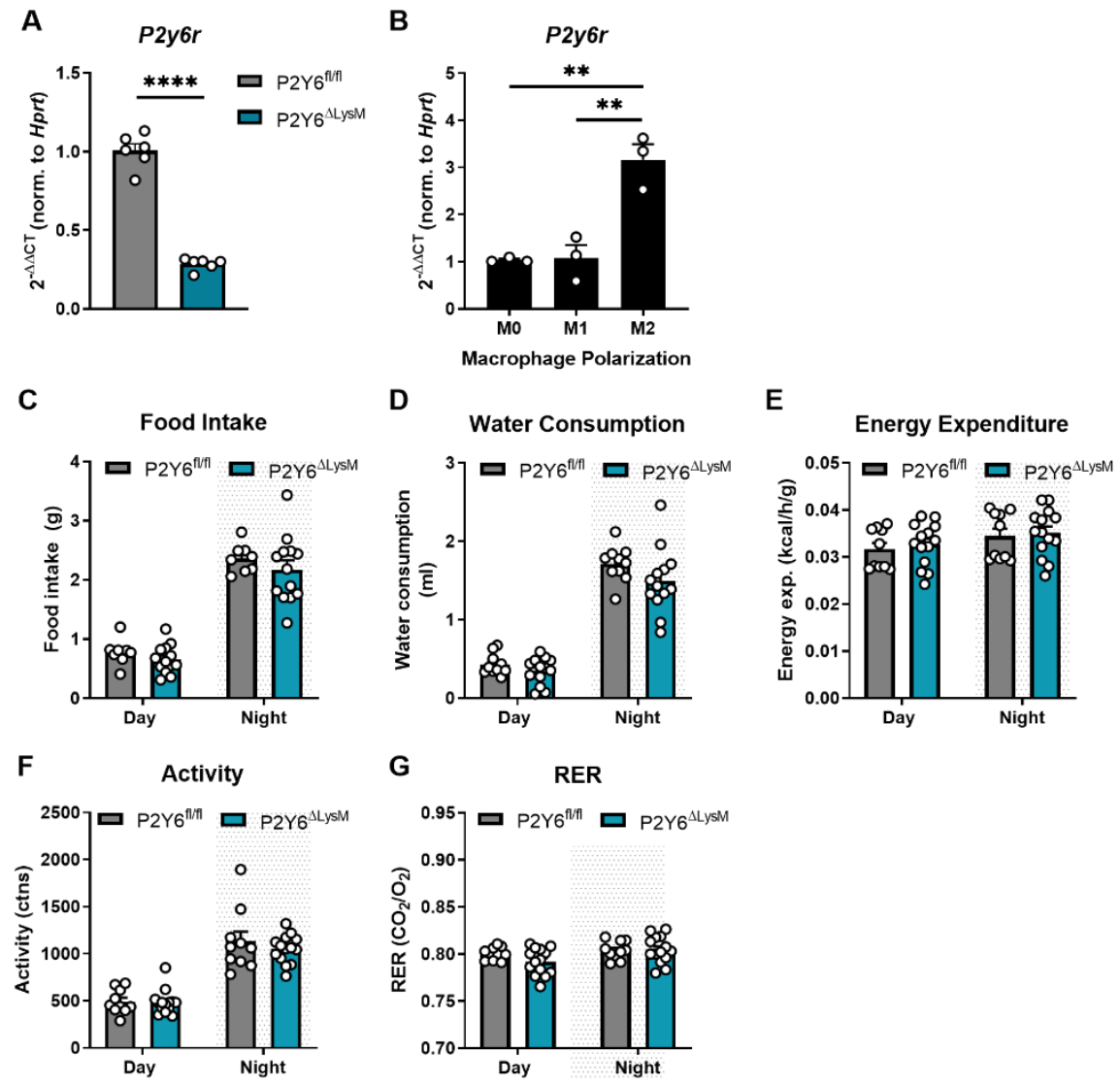

Fig. S9: P2Y6R deletion in macrophages does not alter food intake or energy expenditure. (A) Bone-marrow-derived macrophages were isolated from P2Y6 $6^{\mathrm{fl} / \mathrm{fl}}$ and $\mathrm{P} 2 \mathrm{Y}^{\Delta \mathrm{L}}{ }^{\mathrm{LSM}}$ mice and cultured for 7 days. P2Y6R expression was verified to be reduced in BMDMs of $P 2 Y 6^{\Delta L y s M}$ mice $(n=6)$ compared to control $\left(P 2 Y 6^{f / f / f}, n=6\right)$. Data from two independent experiments. o denotes individual mice. (B) BMDM derived from C57BI6 mice were cultured for 7 days and polarized into M1 or M2 macrophages. P2Y6R expression in M1 and M2 BMDMs was determined by real-time PCR and normalized to the expression in unpolarized (M0) BMDMs. Data pooled from three independent experiments. $\circ$ denotes an individual experiment. (C-G) Indirect calorimetry of mice with macrophage-specific deletion of P2Y6R (P2Y6 $6^{\Delta L y s}$, $n=14$ ) and their control littermates (P2Y $\left.6^{t / f f}, n=10\right)$ after 18 weeks on HFD. $\circ$ denotes individual mice. Statistics: (A) Unpaired two-tailed t-test. ${ }^{* * *} p \leq 0.0001$ (B) Two-way RM ANOVA with Sidak's multiple comparison test. ${ }^{* *} \mathrm{p} \leq 0.01$; (C-G) Unpaired two-tailed student's t-test. Data are represented as mean \pm SEM. 


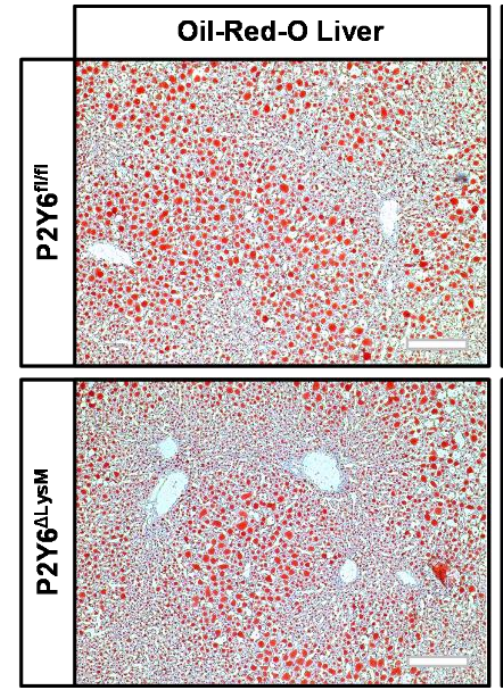

Oil-Red-O Liver

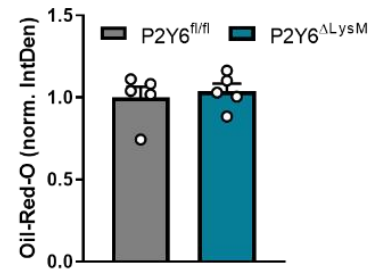

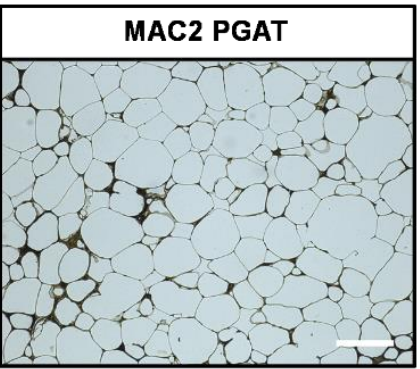

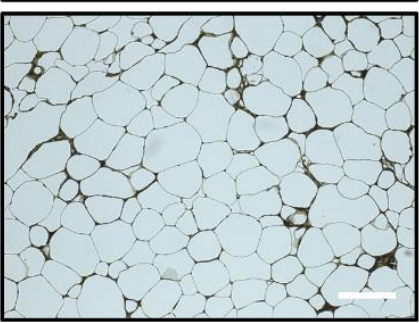

MAC2 PGAT

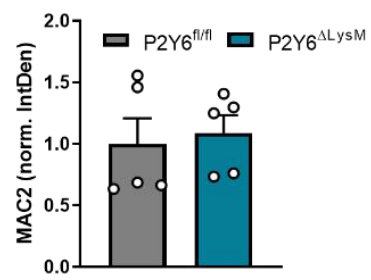

Fig. S10: P2Y6R signaling in macrophages does not affect metaflammation in PGAT of mice on HFD. Transgenic mice (P2Y6 $\left.6^{\Delta L y s M}, n=5\right)$ and their control littermates $\left(P 26^{f / / f t}, n=5\right)$ were fed a HFD for 22 weeks. (left) Oil-Red-O staining of liver tissue. Scale bars $100 \mu \mathrm{m}$. (right) Immunohistochemical analysis of MAC2 positive macrophages in PGAT. Scale bars $100 \mu \mathrm{m}$. Statistics: Unpaired two-tailed ttest. Data are represented as mean \pm SEM. $\circ$ denotes individual mice. 
424 Obesity and its' co-morbidities are often associated with a chronic low-grade

425 inflammation, termed "metaflammation", characterized by increased levels of

426 inflammatory cytokines in serum and infiltration of immune cells into adipose tissue.

427 One immune cell type gaining increasing attention in the context of obesity and glucose

428 metabolism are natural killer cells, a type of innate lymphoid cell (ILC) responsible for

429 surveillance and extinction of virus-infected and tumor cells. In a previous study we

430 reported that P2Y6R expression is up-regulated in NK cells derived from PGAT of HFD-

431 fed mice (Theurich et al., 2017). P2Y6R-signaling has been reported to modulate the

432 production of pro-inflammatory cytokines and chemotaxis of immune cells (Chen et al.,

4332014 ) and to be a relevant regulator of food intake and glucose metabolism in obesity

434 (Steculorum et al., 2015; Steculorum et al., 2017).

$435 \quad$ Here, we demonstrated that UDP and uridine levels are elevated upon HFD

436 feeding. The increase of UDP levels in plasma, adipocytes and adipose tissue-derived

437 SVF after 12 weeks of HFD-feeding coincidences with the development of insulin

438 resistance in wildtype mice. Mice with a NK cell-specific abrogation of P2Y6R signaling

439 were protected from this diet-induced insulin resistance and showed a reduction in the

440 expression of pro-inflammatory chemo- and cytokines in adipose tissue-derived NK

441 cells. NK cells in adipose tissue have been found to induce the infiltration of

442 macrophages mainly by an increased production and release of IFNy (Wensveen et

443 al., 2015), primarily explaining why genetic depletion of NK cells improves adipose

444 tissue inflammation and insulin resistance (Lee et al., 2016). As we did not observe

445 any alterations in the numbers of NK cells but of macrophages and NKT cells in

446 adipose tissue upon NK cell-specific P2Y6R deletion, we rather postulated that the

447 observed reduction in adipose tissue inflammation and associated improvement in

448 insulin sensitivity might be due to a reduced release of inflammatory cytokines from

449 NK cells. And indeed, we found that adipose tissue-derived NK cells showed a

450 decreased gene expression of inflammatory molecules such as $\mathrm{Ccl} 3, \mathrm{Ccl} 4, \mathrm{Xcl} 1$ and

$451 \mathrm{Cxc} / 16$, presumably leading to an attenuation of obesity-induced metaflammation.

452 Obesity-induced metaflammation is a trigger for the development of insulin

453 resistance which is in turn correlated with increased lipolysis in adipose tissue leading

454 to a rise in FFA levels in serum of obese mice and humans (Arner and Rydén, 2015).

455 These FFAs serve as energy source and can, after $\beta$-oxidation, be transformed to ATP

456 by oxidative phosphorylation in mitochondria. Here, we show that NK cell-specific 
457 disruption of P2Y6R-signaling improves insulin sensitivity in mice on HFD, most

458 prominently reflected by an insulin-induced reduction of lipolysis in adipose tissue and

459 repression of glucose production in liver. A causal linkage between insulin suppression

460 of lipolysis and suppression of liver glucose output has been reported in dogs (Rebrin

461 et al., 1996), in rats (Lam et al., 2002; Lam et al., 2003) and in humans (Lewis et al.,

462 1997).

463 In adult liver, expression of the enzyme lipoprotein lipase (Lpl) is physiologically 464 relatively low (Liu et al., 2016) but can be increased by insulin (Ramos et al., 1999) or 465 pro-inflammatory molecules such as TNFa (Enerbäck et al., 1988). We found that $L p l$ 466 gene expression is up-regulated in liver of P2Y6 ${ }^{\Delta N c r 1}$ mice in the clamped (insulin467 stimulated) state but not in basal (unstimulated) conditions (data not shown). 468 Furthermore, it is known, that the activity of Lpl is modulated by apolipoproteins such 469 as apolipoprotein A-V (Apoa5) (Fruchart-Najib et al., 2004) whose expression in turn 470 is regulated by insulin via phosphoinositide 3-kinases (PI3K) (Nowak et al., 2005) or 471 on a transcriptional level by the nuclear receptor RAR Related Orphan Receptor alpha 472 (Rora) (Genoux et al., 2005). In our transcriptomic studies of clamped liver, we found 473 that the expression of both these genes Apoa5 and Rora is down-regulated in $474 \mathrm{P} 2 \mathrm{Y}^{\Delta \mathrm{Ncr} 1}$ mice. These observations further support the finding that hepatic insulin 475 sensitivity is improved in these mice, while insulin-stimulated AKT activation remained 476 unaltered.

477 Collectively, our data show that depletion of P2Y6R in NK cells reduces 478 metaflammation in adipose tissue of HFD-fed mice leading to an overall improvement 479 in insulin sensitivity. This improvement in insulin sensitivity is mainly reflected by a 480 reduction of lipolysis in PGAT, an improved insulin-mediated suppression of HGP and 481 the regulation of insulin-dependent genes, despite the notion that assessment of 482 insulin-stimulated AKT activation upon injection of a high dose of insulin was 483 comparable between the mice of the different genotypes.

$484 \quad$ NK cells are a heterogeneous innate lymphocyte cell population with organ- and 485 microenvironment-specific expression patterns of cytotoxic molecules, receptors and 486 transcription factors (Crinier et al., 2018; Zhao et al., 2020). Despite this tissue487 specificity, we found a common set of ten genes that were similarly differentially 488 expressed in adipose tissue and liver of clamped, thus insulin-stimulated mice. As we 489 assumed that this gene set reflects that of tissue-infiltrating NK cells, rather than being 490 an insulin-regulated gene set, we further investigated the transcriptome of isolated NK 
491 cells from adipose tissue and liver of HFD-fed P2Y6 $6^{\mathrm{fl} / \mathrm{fl}}$ and P2Y6 ${ }^{\Delta \mathrm{Ncr} 1}$ mice. Also here,

492 transcripts of this gene set were found to be differentially expressed in NK cells of both

493 origins, supporting our hypothesis that this gene set is NK cell-specific and that the

494 deletion of P2Y6R from NK cells might alter the intrinsic expression pattern of tissue

495 infiltrating NK cells. There are multiple unique lineages of NK cells, e.g. circulating

496 conventional (c)NK cells, thymic NK cells, tissue-resident (tr)NK cells of the liver and

497 skin and uterine (u)NK cells (Sojka, Tian, Yokoyama, 2014; Peng and Tian, 2017;

498 Hashemi and Malarkannan, 2020). Each of these NK cell populations is characterized

499 by a unique repertoire of receptors and cytokines and seems to emerge from a distinct

500 developmental pathway (Erick and Brossay, 2016). Conventional NK cells are

501 characterized by the expression of DX5 (CD49b) and are found as circulating cells in

502 the blood or as tissue-infiltrating cells in different organs (Sojka et al., 2014; Erick and

503 Brossay, 2016). In contrast to that, tissue-resident NK cells are characterized by the

504 expression of CD49a and exhibit a rather immature phenotype (Sojka, Tian, Yokoyama,

505 2014; Sojka et al., 2014). Despite a large amount of studies with RNASeq data of NK

506 cells from different origins such as blood (Yang et al., 2019; Smith et al., 2020; Zhao

507 et al., 2020), liver (Filipovic et al., 2019; Zhao et al., 2020) and spleen (Peng et al.,

508 2013; Sojka et al., 2014; Zhao et al., 2020), as well as from tumor infiltrating NK cells

509 (de Andrade et al., 2019; Cursons et al., 2019), we could assign none of the ten genes

510 of our NK cell-specific gene cluster to any of these available gene sets with the

511 exception of Rora, whose expression has been reported to be higher in liver-resident

512 DX5-NK cells (Peng et al., 2013).

513 The only gene that was consistently up-regulated in all our transcriptomic data

514 sets was Lilra5. This receptor belongs to the family of leukocyte immunoglobin-like

515 receptors which are known to regulate leukocyte activation. Lilra5 has been reported

516 to exist as transmembrane protein or as secreted molecule (Borges et al., 2003). The

517 ligands for some but not all LILRs are classical and non-classical HLA-class I

518 molecules, however the ligand for Lilra5 is still unknown (Kaur et al., 2013). Cross-

519 linking Lilra5 to the surface of macrophages has been reported to trigger an increase

520 in intracellular calcium concentrations and the release of cytotoxic molecules such as

521 TNFa and IL-10 (Mitchell et al., 2008). Our data shows clear expression of Lilra5 in

522 P2Y6R-deficient NK cells, however in literature it is reported to be mainly expressed in

523 macrophages and to some extend in B cells and granulocytes (Human Protein Atlas,

524 https://www.proteinatlas.org/) and functional studies on Lilra5 until now are limited. 
Collectively, our findings underline the importance of further studies to unravel

526 the role of P2Y6R-signaling in NK cells especially in regard to the regulation of this

527 here reported NK cell-specific gene cluster. In light of the finding that cell numbers in

528 spleen and liver of P2Y6 ${ }^{\Delta \mathrm{Ncr} 1}$ mice are reduced, further studies on conventional and

529 tissue-resident NK cells and their infiltration kinetics in dependence of P2Y6R-signaling

530 should be conducted to further define the impact of NK cell-specific P2YR-signaling on

531 obesity-induced metaflammation.

532 Several publications have reported a role for a P2Y6R-dependent regulation of

533 cytokine expression and release in macrophages (Bar et al., 2008; Lattin et al., 2008).

534 In light of macrophages' roles as drivers for obesity-induced metaflammation (Xu et al.,

535 2003) and an increase in UDP levels in adipose tissue upon HFD-feeding, it was an

536 obvious question whether macrophage-specific P2Y6R-signaling might contribute to

537 obesity-associated metaflammation and development of insulin resistance. However,

538 here we showed that mice with macrophage-specific abrogation of P2Y6R-signaling

539 did not present in any conspicuous phenotype in regard to bodyweight development,

540 insulin sensitivity, food intake or energy expenditure. In addition, we did not observe

541 any alterations in adipose tissue inflammation. As obesity development is

542 accompanied by a macrophage switch from M2- to M1-polarized macrophages in

543 adipose tissue (Castoldi et al., 2016), it was interesting to note that the expression of

$544 \mathrm{P} 2 \mathrm{Y} 6 \mathrm{R}$ is 3-fold up-regulated in M2-polarized macrophages compared to unpolarized

545 or M1-polarized macrophages in vitro. Comparison of M1 and M2 polarized

546 macrophages derived from human blood only reported a difference in the expression

547 of other P2Y receptors such as P2Y1R, P2Y12R and P2Y13R that were found to be

548 down-regulated in M1 compared to M2 macrophages and P2Y2R, P2Y8R, P2Y10R

549 and P2Y14R being up-regulated (Gerrick et al., 2018). Another study on murine

550 BMDMs found P2Y1R to be up-regulated in M2-polarized macrophages when

551 compared to unpolarized cells (Jablonski et al., 2015). Nevertheless, our data clearly

552 indicate that P2Y6R signaling in macrophages appears to be dispensable for the

553 manifestation of obesity-induced metaflammation.

554 Taken together, our study demonstrates that insulin sensitivity and

555 metaflammation depend on P2Y6R signaling in NK cells in the context of obesity, which

556 may provide a target for the treatment of obesity-associated pathologies and diabetes. 


\section{Animals}

561 All mouse experiments were approved by the local authorities (Bezirksregierung Köln,

562 Germany) and conducted in accordance with NIH guidelines. Mice were housed in

563 groups of $3-5$ animals at $22-24^{\circ} \mathrm{C}$ and a 12 -hour light/dark cycle. Mice had ad libitum

564 access to food and water at all times, and food was only withdrawn if required for an

565 experiment. Mouse models were generated by crossing previously published mouse

566 strains, all of which have been backcrossed to C57BL/6 mice for at least ten

567 generations. Briefly, mice with a conditional knockout of the P2Y6R gene in NK cells

568 (P2Y6 ${ }^{\Delta N c r 1}$ ) were generated by crossing Ncr1-Cre mice (Eckelhardt et al., 2011) with

569 P2Y6R-floxed mice (Jain et al., 2020) and line breeding was maintained with Ncr1-

$570 \mathrm{Cre}^{+/-}: \mathrm{P} 2 \mathrm{Y}^{\mathrm{fl} / / f l}$ crossed with Ncr1-Cre ${ }^{-/-}: \mathrm{P} 2 \mathrm{Y}^{\mathrm{fl} / \mathrm{fl}}$ mice. Mice with a conditional knockout

571 of the P2Y6R gene in macrophages (P2Y6 $\left.{ }^{\Delta L y s M}\right)$ were generated by crossing LysM-

572 Cre mice (Clausen et al., 1999) with P2Y6R-floxed mice (Jain et al., 2020) and line

573 breeding was maintained with LysM-Cre ${ }^{+/-}: P 2 Y 6^{f / / f l}$ crossed with LysM-Cre ${ }^{-/-}: P 2 Y 6^{f / f l}$

574 mice. Genotyping of mouse lines was performed by genomic PCR on DNA isolated

575 from tail biopsies using the primers listed in table 1. C57BL/6 N mice were ordered

576 from Charles River (Cologne, Germany).

578 Table 1: Genotyping primers.

\begin{tabular}{llll}
\hline Primer Name & Transgene & Sequence & PCR product \\
\hline Ncr1-Cre227.for & & GACCATGATGCTGGGTTTGGCCCAGATG & \\
Ncr1-Cre146.rev & Ncr1-Cre & ATGCGGTGGGCTCTATGGCTTCTG & Cre: $450 \mathrm{bp}$ \\
Myo F & TTACGTCCATCGTGGACAGC & Ctrl: $650 \mathrm{bp}$ \\
Myi676 & TTCCCGGTATCATCAGCACA & \\
\hline LysM-Cre_1 & LysM-Cre & CTCTAGTCAGCCAGCAGCTG & Cre: $350 \mathrm{bp}$ \\
LysM-Cre_2 & ATGTTTAGCTGGCCCAAATGT & Ctrl: $700 \mathrm{bp}$ \\
\hline P2y6-fl-wt-F & & CTGGGACTTGAACCTAGATCC & wt: $186 \mathrm{bp}$ \\
P2y6-fl-wt-R & P2Y6-fl & GCTACTGCTCCTGTACCTAAGC & flox: $305 \mathrm{bp}$ \\
P2y6del & & GTGCGTCATCGTTGTGTGAGTTTGTGTCGA & del: $370 \mathrm{bp}$ \\
\hline
\end{tabular}




\section{Metabolic Phenotyping}

\section{Diet Induced Obesity and High Fat Diet Feeding}

583 Mice were fed a normal chow diet (NCD) (ssniff R/M-H Phytoestrogenarm, ssniff

584 Spezialdiäten $\mathrm{GmbH}$, Soest, Germany) until used for experiments. Experimental 585 animals were either fed a high-fat diet (HFD) (ssniff D12492 (I) mod., ssniff 586 Spezialdiäten $\mathrm{GmbH}$, Soest, Germany) or a low-fat, low-sugar control diet (CD) (ssniff 587 D12450B mod. LS, ssniff Spezialdiäten $\mathrm{GmbH}$, Soest, Germany) starting at the age of 5886 weeks.

589

\section{Body Composition Analysis}

591 Body weights were assessed weekly. Body composition was analyzed by computed 592 tomography (CT) in isoflurane-anesthetized mice (Dräger Healthcare, Cologne, 593 Germany). For data acquisition on an IVIS Spectrum CT scanner (Caliper LifeScience, 594 Hopkinton, MA, USA) we used IVIS Livinglmage Software V4.3.1. Quantification of 595 lean and fat mass contents were determined with a modification of the previously 596 described Vinci software package 5.06.0 (Cízek et al., 2004) developed at our 597 institution (available at: www.nf.mpg.de/vinci3).

\section{Glucose and Insulin Tolerance Test, and HOMA-IR Determination}

600 Glucose tolerance tests were performed in 16 hours overnight-fasted mice after 9, 14 601 and 20 weeks of HFD- or CD-feeding. Fasted blood glucose concentrations (mg/dl) 602 were determined prior to the i.p. injection of $20 \%(\mathrm{w} / \mathrm{v})$ glucose (B.Braun, Melsungen, 603 Germany; $2 \mathrm{mg} / \mathrm{g}$ body weight). Fifteen, 30, 60 and $120 \mathrm{~min}$ after injection, blood 604 glucose levels in tail vein blood were measured using an automatic glucometer (Bayer 605 Contour Next, Bayer AG, Leverkusen, Germany). Insulin tolerance tests were 606 performed in random fed mice at corresponding ages as GTTs. Blood glucose 607 concentrations $(\mathrm{mg} / \mathrm{dl})$ were determined prior to the test. Before i.p. injection of insulin 608 (0.5 mU/g body weight; Insuman Rapid 40 I.E./ml, Sanofi, Paris, France), food was 609 removed from the cages for the duration of the experiment. Blood glucose levels were 610 measured 15, 30 and 60 min after injection. HOMA-IR is a method to quantify insulin 611 resistance. Here, HOMA-IR was calculated using the formula (Insulin fasted $[\mu U / \mathrm{ml}] \mathrm{x}$ 612 Glucosefasted $[\mathrm{mg} / \mathrm{dl}]) \times 405$. 


\section{Hyperinsulinemic-Euglycemic Clamp Analyses}

616 Surgical implantation of catheters in the jugular vein was performed as previously

617 described (Könner et al., 2007). After one week of recovery, mice were fasted for 4

618 hours in the morning of the day of experiment, which was conducted based on the

619 protocol from the Mouse Metabolomic Phenotyping Center at Vanderbilt University

620 (Ayala et al., 2011). Prior to the actual clamp, mice received $270 \mu \mathrm{l}$ of a $3 \%(\mathrm{v} / \mathrm{v}$ ) plasma

621 solution at a rate of $0.18 \mathrm{ml} / \mathrm{h}$ for $90 \mathrm{~min}$ in parallel with a primed-continuous tracer D-

622 [3- $\left.{ }^{3} \mathrm{H}\right]-$ Glucose infusion (PerkinElmer, Waltham, MA, USA, $0.8 \mu \mathrm{Ci}$ prime, $0.04 \mu \mathrm{Ci} / \mathrm{min}$ ).

623 After this basal period, a blood sample was collected from the tail tip for determination

624 of basal parameters. Following, the clamp started with the infusion of an insulin solution

625 (Sanofi, Paris, France or Lilly, Bad Homburg, Germany; $6 \mu \mathrm{U} / \mathrm{g} / \mathrm{min}$ ) together with a

$62640 \%$ glucose solution (Bela Pharm, Vechta, Germany) primed with D-[3-3 H]-Glucose

$627(0.06 \mathrm{ml} / \mathrm{h})$. After $30 \mathrm{~min}$ of infusion, venous blood glucose levels were determined

628 every 10 min using an ultra-sensitive glucometer (B-Glucose Analyzer; Hemocue,

629 Ängelhom, Sweden) and glucose infusion rates were adjusted in order to keep blood

630 glucose at physiological levels $( \pm 140 \mathrm{mg} / \mathrm{dl})$. The steady state was considered, when

631 a fixed glucose infusion rate maintained the blood glucose levels constant $( \pm 140 \mathrm{mg} / \mathrm{dl})$

632 for $30 \mathrm{~min}$. Approximately $90 \mathrm{~min}$ after start of the insulin infusion, steady state levels

633 were reached. During the steady state, blood samples were collected every 10 min for

634 the measurement of steady-state parameters. Then, animals received a $100 \mu$ bolus

635 of 2-[1-14C]-Deoxy-D-glucose (American Radiolabeled Chemicals Inc., St. Louis, MO,

$636 \mathrm{USA} ; 10 \mu \mathrm{Ci}$ ) at a rate of $1.2 \mathrm{ml} / \mathrm{h}$. Following, infusion was switched back to insulin

$637(6 \mu \mathrm{U} / \mathrm{g} / \mathrm{min})$ and $40 \%$ glucose primed with $\mathrm{D}-\left[3-{ }^{3} \mathrm{H}\right]-G l u c o s e ~(40 \mu \mathrm{l}$ bolus at $1.2 \mathrm{ml} / \mathrm{h}$

638 followed by continuous infusion at the rate used to keep steady state glucose levels).

639 Blood samples were collected and blood glucose levels determined at 0, 5, 10, 15, 25

640 and $35 \mathrm{~min}$ after this last bolus for follow-up determination of 2-[1- $\left.{ }^{14} \mathrm{C}\right]-\mathrm{Deoxy}-\mathrm{D}$ -

641 glucose withdrawal from plasma.

642 At the end of the experiment, mice were sacrificed by cervical dislocation, and liver,

643 perigonodal adipose tissue, brain, brown adipose tissue and skeletal muscle were

644 dissected and stored at $-80^{\circ} \mathrm{C}$. Plasma $\left[3-{ }^{3} \mathrm{H}\right]-$ Glucose radioactivity of basal and

645 steady state was measured as described by the Vanderbilt protocol (Ayala et al., 2011).

646 Plasma 2-[1-14C]-Deoxy-D-glucose radioactivity was directly measured in a liquid

647 scintillation counter (Tri-Carb 2810 TR, Perkin Elmer, Waltham, MA, USA). Tissue

648 lysates were processed through ion exchange chromatography columns (Bio Rad 
649 Laboratories Inc., Hercules, CA, USA) to separate 2-[1-14C]-Deoxy-D-glucose (2DG) 650 from 2-[1-14C]-Deoxy-D-glucose-6-phosphate (2DG6P). Glucose infusion rate (GIR)

651 and hepatic glucose production (HGP) was calculated as previously described (Könner

652 et al., 2007). In vivo glucose uptake into perigonodal and brown adipose tissue, brain,

653 liver and skeletal muscle was calculated based on the accumulation of 2DG6P in the

654 respective tissue and the disappearance rate of 2DG from plasma as described

655 previously (Könner et al., 2007).

656

\section{Analyses of Plasma Samples}

658 Blood was collected in EDTA-treated tubes (Sarstedt, Nümbrecht, Germany) and 659 centrifuged for $10 \mathrm{~min}$ at $17000 \mathrm{~g}$ and $4^{\circ} \mathrm{C}$. The plasma was immediately frozen in 660 liquid nitrogen and stored at $-80^{\circ} \mathrm{C}$ until used for ELISA analysis. Fasted mouse insulin 661 and leptin plasma concentrations were determined by ELISA with mouse standards 662 according to the manufacturer's guidelines (Ultra Sensitive Mouse Insulin ELISA Kit, 663 Crystal Chem, Elk Grove Village, IL, USA; Mouse Leptin ELISA Kit, Crystal Chem, Elk 664 Grove Village, IL, USA). For the leptin ELISA, plasma samples were diluted 1:20, for 665 the insulin ELISA undiluted plasma samples were used. The concentration of human 666 insulin in plasma of clamped mice was determined by ELISA according to 667 manufacturer's guidelines (Insulin (Human) Ultrasensitive, DRG Diagnostics, Marburg, 668 Germany) using 1:25 diluted plasma samples. Free fatty acid concentration in plasma 669 (diluted 1:25) of clamped mice was determined using a quantitative Free Fatty Acid 670 Assay Kit (Abcam, Cambridge, Great Britain). Absorbance was measured with the 671 Filter Max F5 device (Molecular Devices, San José, CA, USA) at corresponding 672 wavelengths. Concentrations were calculated using a 4-parameter logistic regression 673 analysis.

674

\section{Indirect Calorimetry}

676 Indirect calorimetry was performed using an open-circuit, indirect calorimetry system 677 (PhenoMaster, TSE systems, Bad Homburg vor der Höhe, Germany). Three days 678 before data acquisition, mice were adapted to the food/drink dispensers of the 679 PhenoMaster system and the body weight was monitored. Then, mice were placed into 680 regular type II cages with sealed lids and allowed to adapt to the chambers at least 24 681 hours at room temperature $\left(22^{\circ} \mathrm{C}\right)$. During data acquisition, mice were provided with 
682 food and drink ad libitum. All parameters were measured simultaneously and 683 continuously.

684

\section{Gene Expression Analyses}

\section{RNA Extraction from Tissues and Cells}

687 Snap frozen tissue samples or cells were homogenized in 0.5-1 ml Qiazol (Qiagen, 688 Hilden, Germany) using a Retsch Homogenizer (Retsch GmbH, Haan, Germany) 689 combined with beads (Bertin Technologies, Montigny-le-Bretonneux, France). 690 Afterwards, 0.2-0.4 $\mathrm{ml}$ chloroform were added to each sample and the mixture 691 incubated for $5 \mathrm{~min}$ at RT. Following, samples were centrifuged for $15 \mathrm{~min}$ at $4^{\circ} \mathrm{C}$ and $69212200 \mathrm{~g}$. The supernatant was transferred to a new tube and mixed with the equal 693 volume of $70 \%$ ethanol. The total volume was then transferred to a RNeasy Mini Spin 694 Column of the RNeasy Mini Kit (Qiagen, Hilden, Germany). Subsequent steps were 695 conducted as described in the manufacturer's protocol. DNAse treatment (15 min at 696 RT; Qiagen, Hilden, Germany) was only conducted in samples used for RNASeq but 697 not in samples used for qPCR analysis. Finally, RNA was eluted in $\mathrm{H}_{2} \mathrm{O}$ and stored at $698-80^{\circ} \mathrm{C}$ until further use.

699

\section{cDNA Synthesis and Quantitative Real Time PCR}

701 Concentrations of RNA extracted from PGAT, liver or BMDMs were determined with 702 the NanoDrop ND-1000 device (Thermo Fisher Scientific, Waltham, MA, USA). cDNA 703 synthesis was conducted using the High Capacity cDNA RT kit according to 704 manufacturer's guidelines (Applied Biosystems, Foster City, CA, USA). Following, 705 cDNA was used for real-time PCR analysis using Takyon Low Rox Probe 2x Master 706 Mix dTTP Blue (Eurogentec, Liège, Belgium) with TaqMan® Assay-on-demand kits 707 (Applied Biosystems, Foster City, CA, USA). Relative expression of target mRNAs was 708 adjusted for total RNA content by hypoxanthine guanine phosphoribosyl transferase 1 709 (Hprt1) or TATA binding protein (Tbp) mRNA qRT-PCR (for target probe details see 710 table 2). Calculations were performed by a comparative method (2- $\Delta \Delta \mathrm{CT})$. Quantitative 711 PCR was performed on a QuantStudio 7 Flex Real-Time PCR System using the

712 QuantStudio Real-Time PCR Software v1.3 (Life Technologies, Carlsbad, CA, USA). 
716 Table 2: Probes used for quantitative real time PCR.

\begin{tabular}{llll}
\hline Target gene & Probe ID & Fluorophore & Company \\
\hline P2y6r & Mm02620937_s1 & FAM & Thermo Fisher Scientific \\
II6 & Mm00446190_m1 & FAM & Thermo Fisher Scientific \\
Tnfa & Mm00443258_m1 & FAM & Thermo Fisher Scientific \\
Cc/2 & Mm00441242_m1 & FAM & Thermo Fisher Scientific \\
Cc/3 & Mm00441258_m1 & FAM & Thermo Fisher Scientific \\
Ifng & Mm01168134_m1 & FAM & Thermo Fisher Scientific \\
II10 & Mm00439614_m1 & FAM & Thermo Fisher Scientific \\
II12a & Mm00434165_m1 & FAM & Thermo Fisher Scientific \\
II1b & Mm01336189_m1 & FAM & Thermo Fisher Scientific \\
Lpl & Mm00434764_m1 & FAM & Thermo Fisher Scientific \\
Rora & Mm01173766_m1 & FAM & Thermo Fisher Scientific \\
Plin2 & Mm00475794_m1 & FAM & Thermo Fisher Scientific \\
Lilra5 & Mm01335361_m1 & FAM & Thermo Fisher Scientific \\
Hprt & Mm01545399_m1 & FAM & Thermo Fisher Scientific \\
Hprt & Mm03024075_m1 & VIC & Thermo Fisher Scientific \\
\hline Tbp & Mm00446973_m1 & VIC & Thermo Fisher Scientific \\
\hline
\end{tabular}

717

\section{RNA Sequencing of Clamped Tissue Samples}

719 RNA from clamped liver and PGAT samples was isolated as described above. Libraries 720 were prepared using the TruSeq Stranded mRNA protocol according to the

721 manufacturer's recommendations (Illumina, San Diego, CA, USA). After validation with

722 the Agilent 4200 TapeStation (Agilent, Santa Clara, CA, USA) and quantification with

723 a Qubit device (Invitrogen, Carlsbad, CA, USA), all transcriptome libraries were pooled.

724 The pool was quantified using the Peqlab KAPA Library Quantification Kit (VWR,

725 Radnor, PA; USA) and the Applied Biosystems 7900HT Sequence Detection

726 (ThermoFisher, Waltham, MA, USA) and sequenced on a Illumina NovaSeq S2 flowcell

727 (Illumina, San Diego, CA, USA) with a PE100 protocol. 


\section{Immunoblots}

\section{Protein Extraction from Tissue Samples}

732 Snap frozen tissue samples were homogenized in $1 \mathrm{ml}$ lysis buffer containing $50 \mathrm{mM}$

733 Tris, $130 \mathrm{mM} \mathrm{NaCl}, 5 \mathrm{mM}$ EDTA, 1\% (v/v) NP-40, 1 mM NaF, 1 mM PMSF (Sigma

734 Aldrich, St. Louis, MO, USA), cOmplete mini protease inhibitor cocktail (1 tablet/10 ml;

735 Roche, Basel, Switzerland) and PhosSTOP Phosphatase Inhibitor Cocktail (1 tablet/10

$736 \mathrm{ml}$; Roche, Basel, Switzerland) using a Retsch Homogenizer (Retsch GmbH, Haan,

737 Germany) combined with ceramic beads (Bertin Technologies, Montigny-le-

738 Bretonneux, France). After homogenization for $2 \mathrm{~min}$ at a frequency of $30 \mathrm{1} / \mathrm{s}$,

739 homogenates were incubated for $20 \mathrm{~min}$ on ice followed by centrifugation at

$74013000 \mathrm{rpm}$ for $20 \mathrm{~min}$ at $4^{\circ} \mathrm{C}$. Protein concentration was determined relative to a BSA

741 standard using the Pierce ${ }^{T M}$ BCA $^{T M}$ Protein-Assay (Thermo Scientific, Waltham, MA,

742 USA). Lysates were finally adjusted to a final concentration of $2 \mu \mathrm{g} / \mu \mathrm{l}$ with $4 \mathrm{X}$ Laemmli

743 buffer (Bio-Rad, Hercules, CA, USA) containing 10\% $\beta$-Mercaptoethanol.

\section{Western Blotting}

746 Proteins (20 $\mu \mathrm{g}$ per well) were separated in 4-15\% Criterion ${ }^{\mathrm{TM}}$ TGX ${ }^{\mathrm{TM}}$ Precast Midi 747 Protein Gels with 26 wells (Bio-Rad, Hercules, CA, USA) at a constant voltage of 748120 V. The SDS-Running buffer contained $200 \mathrm{mM}$ Glycine, $25 \mathrm{mM}$ Tris and 1\% (w/v) 749 SDS. Proteins were transferred to Trans-Blot Turbo Midi $0.2 \mu \mathrm{m}$ PVDF Transfer 750 membranes (Bio-Rad, Hercules, CA, USA) using the Trans-Blot Turbosystem (Bio-Rad, 751 Hercules, CA, USA). Following, membranes were blocked in western blotting reagent 752 (Roche, Basel, Switzerland) diluted 1:10 in TBS-T buffer (Tris-buffered saline with 1\% $753(\mathrm{v} / \mathrm{v})$ Tween 20$)$ for $1-2 \mathrm{~h}$ at room temperature. Incubation with primary antibodies was 754 conducted over night at $4^{\circ} \mathrm{C}$. Subsequently, membranes were washed three times for 755 5-7 $\mathrm{min}$ in TBS-T followed by the incubation in horse radish peroxidase-labeled 756 secondary antibody over night at $4^{\circ} \mathrm{C}$. All primary and secondary antibodies were 757 diluted in 5\% (v/v) western blotting reagent (Roche, Basel, Switzerland). After washing 758 for 3 times in TBS-T, membranes were incubated with SuperSignal ECL Western

759 Blotting Substrate (Thermo Fisher Scientific, Waltham, MA, USA) and luminescence 760 was detected by the Fusion Solo Vilber Lourmat system (Vilber Lourmat $\mathrm{GmbH}$, 761 Eberhardzell, Germany). If necessary, membranes were stripped in stripping buffer 762 (6\% SDS (v/v), $188 \mathrm{mM}$ Tris $\mathrm{pH} 6.8,2 \%$ (v/v) $\beta$-Mercaptoethanol) for $30 \mathrm{~min}$ at $56^{\circ} \mathrm{C}$ 763 in a shaking water bath. Afterwards, membranes were washed three times in TBS-T 
764 followed by blocking in $10 \%$ (v/v) western blotting reagent (Roche, Basel, Switzerland)

765 for $1-2 \mathrm{~h}$ at room temperature. Then, the incubation with primary and secondary

766 antibodies was conducted as described above. Band intensities were quantified using

767 Image $(\mathrm{NIH})$. The intensity of protein bands was normalized to the intensity of calnexin

768 or $\beta$-Actin bands which served as loading control. Band intensities of protein bands

769 from phospho-targets such as pAKT and pHSL were additionally normalized to bands

770 of total protein, AKT and HSL, respectively. For detailed information of individual

771 primary and secondary antibodies see table 3.

772

773 Table 3: Detailed description of antibodies used for Western Blots.

\begin{tabular}{lccll}
\hline Target & Host & Dilution & Company & Order No. \\
\hline pAKT & rabbit & $1: 2000$ & Cell Signaling, Danvers, MA, USA & \#4060 \\
AKT & rabbit & $1: 2000$ & Cell Signaling, Danvers, MA, USA & $\# 4685$ \\
Calnexin & rabbit & $1: 4000$ & Merck kGaA, Darmstadt, Germany & $\# 208880$ \\
B-Actin & rabbit & $1: 1000$ & Cell Signaling, Danvers, MA, USA & $\# 4967$ \\
pHSL-Ser563 & rabbit & $1: 1000$ & Cell Signaling, Danvers, MA, USA & $\# 4139$ \\
pHSL-Ser565 & rabbit & $1: 1000$ & Cell Signaling, Danvers, MA, USA & $\# 4137$ \\
HSL & rabbit & $1: 1000$ & Cell Signaling, Danvers, MA, USA & $\# 4107$ \\
Anti-rabbit-HRP & goat & $1: 2000$ & Sigma Aldrich, St. Louis, MO, USA & \#A6154
\end{tabular}

774

775 Analyses of Isolated Immune Cells

776 Immune Cell Isolation from Tissue

777 Leucocytes were isolated from perigonodal adipose tissue (PGAT), liver, spleen and

778 blood from HFD-fed male mice according to the protocol described in Theurich et al.,

779 2017. Briefly, liver and PGAT were dissociated in lysis buffer (liver: $150 \mathrm{mM} \mathrm{NaCl}, 5.6$

$780 \mathrm{mM} \mathrm{KCl}, 5.5 \mathrm{mM}$ Glucose, $20.1 \mathrm{mM}$ HEPES, 25 mM NaHCO , $2 \mathrm{mM} \mathrm{CaCl}$, 2 mM

$781 \mathrm{MgCl}_{2}, 500 \mathrm{U} / \mathrm{ml}$ Collagenase IV; PGAT: 120 mM NaCl, 5.2 mM KH $\mathrm{PO}_{2}, 1$ mM MgSO ,

$78210 \mathrm{mM}$ Hepes, $10 \mathrm{mM} \mathrm{NaHCO} 3,500 \mathrm{U} / \mathrm{ml}$ Collagenase I) with a tissue-lyser (Gentle

783 MACS, Miltenyi Biotec, Bergisch Gladbach, Germany) and digested enzymatically for

784 20-30 $\min$ at $37^{\circ} \mathrm{C}$ while continuously shaking in a water bath. The spleen was

785 dissociated by pressing it through a $100 \mu \mathrm{m}$ nylon mesh (Greiner Bio-One, Solingen,

786 Germany) and cells were collected in FACS buffer (autoMACS Running Buffer, Miltenyi

787 Biotec, Bergisch Gladbach, Germany). Blood was collected in EDTA-treated tubes 
788 (Sarstedt, Nümbrecht, Germany). Immune cells were separated by centrifugation:

789 adipose tissue homogenates at $400 \mathrm{~g}$ and $18^{\circ} \mathrm{C}$ for $5 \mathrm{~min}$, liver homogenates via $20 \%$

790 (w/v) Histodenz (Sigma Aldrich, St. Louis, MO, USA) density-centrifugation at $1500 \mathrm{~g}$

791 and $4^{\circ} \mathrm{C}$, spleen homogenates at $400 \mathrm{~g}$ and $4^{\circ} \mathrm{C}$ for $5 \mathrm{~min}$. Following, cells and blood

792 were subjected to erythrocyte lysis in RBC lysis buffer (BioLegend, San Diego, CA,

793 USA) for 10 min on ice. Erythrocyte lysis was terminated by washing the cells in the

794 10-fold volume of FACS buffer (autoMACS Running Buffer, Miltenyi Biotec, Bergisch

795 Gladbach, Germany) and passed through a $30 \mu \mathrm{m}$ cell strainer (BD Biosciences,

796 Franklin Lakes, USA) to remove large cellular debris. After centrifugation at $400 \mathrm{~g}$ and

$7974^{\circ} \mathrm{C}$ for $5 \mathrm{~min}$, cells were resuspended in $1 \mathrm{ml} \mathrm{FACS}$ buffer (autoMACS Running Buffer,

798 Miltenyi Biotec, Bergisch Gladbach, Germany) and used for cell counting.

799

\section{Flow Cytometry}

801 For flow cytometry analysis, $1 \times 10^{5}-1 \times 10^{6}$ cells were stained after Fc-blocking with

802 TruStain FcX (BioLegend, San Diego, CA, USA) and incubation with Zombie Aqua

803 fixable viability dye (BioLegend, San Diego, CA, USA). Fluorochrome-conjugated

804 antibodies were used for specific immunostainings with dilutions (in autoMACS

805 Running Buffer, Miltenyi Biotec, Bergisch Gladbach, Germany) as listed in table 4: anti806 mCD45 (30-F11), anti-mCD3 (17A2), anti-mCD19 (6D5), anti-mNK1.1 (PK136), anti807 mNcr1 (29A1.4), anti-mCD11b (M1/70), anti-mCD27 (LG.3A10) and anti F4/80 (BM8).

808 Cells were analyzed using an 8-color flow cytometer (MACSQuant10, Miltenyi Biotec,

809 Bergisch Gladbach, Germany) and data analysis was performed with FlowJo 10.4

810 (FlowJo LLC, BD Biosciences, San Jose, CA, USA) software packages.

811

812 
813 Table 4: Detailed description of antibodies used for flow cytometry.

\begin{tabular}{llllll}
\hline Target & Fluorophor & Dilution & Clone & Company & Oder No. \\
\hline NK1.1 & BV421 & $1: 50$ & PK136 & BioLegend, San Diego, CA, USA & $\# 108732$ \\
CD19 & PE/Cy7 & $1: 50$ & 6 D5 & BioLegend, San Diego, CA, USA & $\# 115520$ \\
CD3 & APC & $1: 100$ & $17 A 2$ & BioLegend, San Diego, CA, USA & $\# 100236$ \\
CD45 & APC/Cy7 & $1: 100$ & $30-F 11$ & BioLegend, San Diego, CA, USA & $\# 103115$ \\
CD11b & PerCP/Cy5.5 & $1: 100$ & M1/70 & BioLegend, San Diego, CA, USA & $\# 101227$ \\
CD27 & PE/Cy7 & $1: 50$ & LG.3A10 & BioLegend, San Diego, CA, USA & $\# 124215$ \\
Live/Dead ZombieAqua & $1: 100$ & - & BioLegend, San Diego, CA, USA & $\# 423102$ \\
F4/80 & PE & $1: 50$ & BM8 & BioLegend, San Diego, CA, USA & $\# 123110$ \\
Ncr1 & BV421 & $1: 20$ & $29 A 1.4$ & BioLegend, San Diego, CA, USA & $\# 137611$ \\
NK1.1 & Alexa488 & $1: 50$ & PK136 & BioLegend, San Diego, CA, USA & $\# 108718$ \\
TruStain & - & $1: 100$ & - & BioLegend, San Diego, CA, USA & $\# 101320$ \\
FCX & & & & & \\
CD3 & PerCP/Cy5.5 & $1: 100$ & $17 A 2$ & BioLegend, San Diego, CA, USA & $\# 100218$ \\
CD11b & APC & $1: 100$ & M1/70 & BioLegend, San Diego, CA, USA & $\# 101212$ \\
& & & & &
\end{tabular}

814

\section{RNA Isolation and RNASeq of Bulk Sorted NK Cells}

816 NK cells from murine organs and blood were purified from single cell suspensions

817 using FACSAria Illu or FACSAria Fusion cell sorters (BD Biosciences, San Jose, CA,

818 USA) after immunostainings as described above. NK cells were sorted from HFD-fed

819 animals identified as single/viable/CD45 $/$ CD3-/NK1.1 ${ }^{+} / \mathrm{Ncr} 1^{+}$cells using a $70 \mu \mathrm{m}$

820 nozzle at 70 psi pressure. During sorting, sample and collection tubes were chilled.

821 Purified cells were directly sorted into FACS buffer (autoMACS Running Buffer, Miltenyi

822 Biotec, Bergisch Gladbach, Germany) and pelleted by centrifugation for 5 min at

$823400 \mathrm{~g}$ and $4^{\circ} \mathrm{C}$. Total RNA was extracted using the Arcturus RNA picopure kit (Thermo

824 Fisher Scientific, Waltham, MA, USA) following the manufacturer's instructions. Due to

825 the low amount of input material, pre-amplification using the Ovation RNASeq System

826 V2 (NuGEN, Redwood, CA, USA), was performed as described previously (Paeger et

827 al., 2017). For library preparation, the Illumina Nextera XT DNA sample preparation

828 protocol (Illumina, San Diego, CA, USA) was used, with 1 ng cDNA input. After 
829 validation with the Agilent 4200 TapeStation (Agilent, Santa Clara, CA, USA) and

830 quantification with a Qubit device (Invitrogen, Carlsbad, CA, USA) all libraries were

831 pooled. The pool was quantified using the Peqlab KAPA Library Quantification Kit

832 (VWR, Radnor, PA; USA) and the Applied Biosystems 7900HT Sequence Detection

833 (ThermoFisher, Waltham, MA, USA) and sequenced on an Illumina NovaSeq system

834 (Illumina, San Diego, CA, USA) with PE100 read length.

835

\section{Analysis of RNA-Sequencing Results}

837 We applied the community-curated nfcore/rnaseq analysis pipeline version 1.4 .2 838 (Ewels et al., 2019) for processing RNA-sequencing data. The gene-level 839 quantification was carried out using Salmon 0.14.1 (Patro et al., 2017) using the

840 reference genome GRCm38. The differential gene expression analysis was done using 841 the DESeq2 1.26.0 (Love et al., 2014) R package. We denote a gene differentially 842 expressed with an FDR adjusted_p_value $\leq 0.05$. Gene-ontology term analysis of the 843 differentially expressed genes was carried out using the clusterProfiler 3.14.3 (Yu et 844 al., 2012) R package.

845

\section{NK Cell Isolation by Magnetic Separation and DNA Extraction}

847 For knock out validation, NK cells were isolated from spleen of P2Y6 ${ }^{f / f l}$ and P2Y6 ${ }^{\Delta N c r 1}$ 848 mice fed a normal chow diet using the mouse NK cell isolation kit from Miltenyi Biotec, 849 (Bergisch Gladbach, Germany). The spleen was dissociated by pressing it through a $850100 \mu \mathrm{m}$ nylon mesh (Greiner Bio-One, Solingen, Germany). Following, erythrocyte 851 lysis was carried out for 10 min on ice in RBC lysis buffer (BioLegend, San Diego, CA, $852 \mathrm{USA}$ ). Cells were centrifuged for $5 \mathrm{~min}$ at $400 \mathrm{~g}$ and $4^{\circ} \mathrm{C}$. The pellet was resuspended 853 in FACS buffer (autoMACs Running Buffer, Miltenyi Biotec, Bergisch Gladbach, 854 Germany) and cells were passed through a $30 \mu \mathrm{m}$ nylon mesh to remove cell clumps.

855 After a second centrifugation at $400 \mathrm{~g}$ for $5 \mathrm{~min}$ at $4^{\circ} \mathrm{C}$, cells were resuspended in $1 \mathrm{ml}$ 856 FACS buffer (autoMACS Running Buffer, Miltenyi Biotec, Bergisch Gladbach, 857 Germany) and used for cell counting. Following, NK cells were isolated according to 858 the manufacturer's protocol (Miltenyi Biotec, Bergisch Gladbach, Germany). As 3 x $10^{7}$ 859 cells were used per sample, all reagent volumes and total volumes were scaled up 860 accordingly. Magnetic separation was carried out using LS columns (Miltenyi Biotec, 861 Bergisch Gladbach, Germany). 
862 After separation, DNA was extracted from isolated NK cells and cells of the flow

863 through (nonNK cells). Cells were lysed in lysis buffer (100 mM Tris-HCl pH 8.5, 5 mM

864 EDTA pH 8.0, 0.2\% SDS (v/v), $200 \mathrm{mM} \mathrm{NaCl}$ ) at $37^{\circ} \mathrm{C}$ over night. DNA was precipitated

865 in $0.5 \mathrm{ml}$ ice-cold isopropanol followed by incubation in $0.5 \mathrm{ml} 70 \%$ ethanol. The DNA

866 pellet was resuspended in $\mathrm{H}_{2} \mathrm{O}$ and used for genomic PCR with primers listed in

867 table 1.

868

869 Seahorse Analyses of Liver Micro-Punches

870 To obtain consistent liver punches for analysis, we used a $0.5 \mathrm{~mm}$ EMS-Core Sampling

871 Tool (Electron Microscopy Science, Hatfield, PA, USA). Four to five tissue punches per

872 animal were carefully moved into Seahorse XF96e spheroid microplates (Agilent,

873 Santa Clara, CA, USA) containing Seahorse base medium (Agilent, Santa Clara, CA,

874 USA) with $25 \mathrm{mM}$ glucose, $1 \mathrm{mM}$ sodium pyruvate and $2 \mathrm{mM}$ L-glutamine. The

875 spheroid microplates (Agilent, Santa Clara, CA, USA) were coated with Cell-Tak

876 (22.4 mg/mL; Corning, Corning, NY, USA) to prevent detachment of the tissue punches

877 during the assay. Plates were then transferred to a $\mathrm{CO}_{2}$-free incubator and maintained

878 at $37^{\circ} \mathrm{C}$ for 30 min before starting the assay. Following instrument calibration, plates

879 were transferred to the Seahorse XF96 Flux Analyzer (Agilent, Santa Clara, CA, USA)

880 to record cellular oxygen consumption rates (OCR) and extracellular acidification rates

881 (ECAR). Ten measurements were performed to assess baseline levels.

882

883 Histological Analysis of PGAT and Liver

884 MAC2 Staining in PGAT

885 Perigonodal adipose tissue (PGAT) was isolated and fixed in $4 \%$ (w/v) 886 paraformaldehyde for $2-3$ days at $4^{\circ} \mathrm{C}$. Fixed tissue samples were then embedded in 887 paraffin and $5 \mu \mathrm{m}$ thick sections were cut at a rotary microtome (Leica, Wetzlar, 888 Germany). After a 10 min incubation in PBS, endogenous peroxidases were blocked 889 for 20 min with $0.3 \%(v / v) \mathrm{H}_{2} \mathrm{O}_{2}$ (in PBS and 0.25\% (v/v) TritonX). After washing with 890 PBS, sections were blocked with 10\% (v/v) Rotiblock (Carl Roth, Karlsruhe, Germany) 891 (diluted in PBS and 0.25\% (v/v) TritonX) for $80 \mathrm{~min}$. Following, primary antibody 892 incubation in rat-anti-MAC2 antibody (Biozol, Eching, Germany; 1:100 diluted in 893 Signalstain, Cell Signaling, Danvers, MA, USA) was conducted overnight at $4^{\circ} \mathrm{C}$. After 894 washing with PBS and $0.25 \%$ (v/v) Triton X, secondary antibody incubation with HRP895 conjugated goat-anti-rat (Jackson Immuno Research, Cambridgeshire, UK; 1:100 
896 diluted in PBS $+0.1 \%(v / v)$ TritonX) was carried out for $1 \mathrm{~h}$ at room temperature. After

897 washing in PBS and $0.25 \%$ Triton $X$ followed by DAB reaction and staining of nuclei

898 via Mayers Hematoxylin (Sigma Aldrich, St. Louis, MO, USA), sections were scanned

899 on a Leica DM 1000 LED (Leica, Wetzlar, Germany).

900

\section{Oil-Red-O Staining in Liver}

902 After sacrifice, liver tissue was embedded in tissue freezing medium (Leica, Wetzlar,

903 Germany) and frozen down on dry ice. Liver sections were cut on a cryostat (Leica,

904 Wetzlar, Germany) as $7 \mu \mathrm{m}$ thick sections. Prior to the staining, tissue sections were

905 washed for $5 \mathrm{~min}$ in tap water, followed by an incubation in Oil-Red-O solution (0.3 g

906 Oil-Red-O (Sigma Aldrich, St. Louis, MO, USA) in $60 \mathrm{ml}$ isopropanol and $40 \mathrm{ml}$ tap

907 water) for $15 \mathrm{~min}$. After several washes in tap water, sections were incubated for $5 \mathrm{~min}$

908 in Mayers Hematoxylin (Sigma Aldrich, St. Louis, MO, USA) followed by a 15 min

909 incubation in tap water. Sections were embedded in Kaiser's glycerine gelatine (Merck

910 Millipore, Burlington, MA, USA) and scanned on a Leica DM 1000 LED (Leica, Wetzlar,

911 Germany).

912

\section{Quantification of Immunohistochemical Stainings}

914 MAC2 and Oil-Red-O stainings were quantified using Image J (NIH, Bethesda, MD,

915 USA). First, images (RGB color) were split into different channels. Threshold was

916 adjusted for the blue channel by finding a threshold value that fits at minimum three

917 independent pictures. Integrated density values were used in order to compare

918 genotypes. Each data point in Suppl. Fig.S8A and Suppl. Fig. S10 represents the mean

919 integrated density value \pm SEM of 2-3 technical replicates each of them originating

920 from one biological replicate (one mouse).

921 The size of adipocytes was quantified using the Adiposoft plugin (Version 1.16; CIMA,

922 University of Navarra) for Image J (NIH, Bethesda, MD, USA).

\section{Bone Marrow-Derived Macrophage Cultures}

925 Bone marrow was isolated from ethanol-rinsed (70\% v/v) femurs and tibias of C57BL/6,

$926 \mathrm{P} 2 \mathrm{Y}^{\Delta \mathrm{LysM}}$ or P2Y6 ${ }^{\mathrm{fl} / \mathrm{fl}}$ mice fed a normal chow diet. Collected cells were centrifuged for

$9271 \mathrm{~min}$ at $300 \mathrm{~g}$ and $4^{\circ} \mathrm{C}$, followed by red blood cell lysis in RBC lysis buffer (BioLegend,

928 San Diego, CA, USA) for $5 \mathrm{~min}$ on ice. Following, cells were passed through a $30 \mu \mathrm{m}$

929 cell strainer to remove cellular debris and pelleted by centrifugation at $1200 \mathrm{rpm}$ for 
$9305 \mathrm{~min}$ at $4^{\circ} \mathrm{C}$. Then, cells were re-suspended in culture medium (RPMI $1640 \mathrm{w} / \mathrm{O}$

931 phenolred (Thermo Fisher Scientific, Waltham, MA, USA) supplemented with 10\% FCS

$932(\mathrm{v} / \mathrm{v}), 1 \%$ glutamine (v/v), 1\% penicillin-streptomycin (v/v) and $50 \mathrm{ng} / \mathrm{ml} \mathrm{M}-\mathrm{CSF}$

933 (Miltenyi Biotec, Bergisch Gladbach, Germany) and seeded at a density of $5 \times 10^{5}$ cells

934 per well of a 6-well plate. Cells were differentiated for a minimum of 7 days with medium

935 exchange every third day. One day before polarization experiments, BMDMs were

936 washed twice with sterile PBS and further cultured in the absence of M-CSF. Cells

937 were stimulated with $100 \mathrm{ng} / \mathrm{ml}$ LPS (Sigma Aldrich, St. Louis, MO, USA) and $20 \mathrm{ng} / \mathrm{ml}$

938 IFNy (Thermo Fisher Scientific, Waltham, MA, USA) for M1-polarization or with

$93950 \mathrm{ng} / \mathrm{ml}$ IL-6 (R\&D Systems, Minneapolis, MN, USA) and $20 \mathrm{ng} / \mathrm{ml}$ IL-4 (Miltenyi

940 Biotec, Bergisch Gladbach, Germany) for M2-polarization. After $24 \mathrm{~h}$ of stimulation,

941 cells were washed twice with sterile PBS and harvested using cell scarpers. Following

942 centrifugation, cell pellets were stored at $-80^{\circ} \mathrm{C}$ until further use.

943

944 Lipidomic Analyses of Liver Tissue

945 Chain-length specific DAG and TAG species from liver samples were quantified by

946 nano-electrospray ionization tandem mass spectrometry (Nano-ESI-MS/MS)

947 according to the protocol described in Turpin et al. 2019. Conditions of lipid extraction,

948 Nano-ESI-MS/MS analysis and processing of mass spectra and quantification of lipid

949 species was done as previously described (Kumar et al., 2015).

950

951 Metabolomic Analyses

952 Sample collection

953 Mice were sacrificed after 6 and 12 weeks of HFD- or normal chow-diet feeding. A

954 piece of liver tissue from the left lobe was dissected and directly snap frozen in liquid

955 nitrogen. Perigonodal adipose tissue was dissected and dissociated in lysis buffer (120

$956 \mathrm{mM} \mathrm{NaCl}, 5.2 \mathrm{mM} \mathrm{KH} \mathrm{PO}_{2}, 1 \mathrm{mM} \mathrm{MgSO} 4,10 \mathrm{mM}$ Hepes, $10 \mathrm{mM} \mathrm{NaHCO}$, 500U/ml

957 Collagenase I) with a tissue-lyser (Gentle MACS, Miltenyi Biotec, Bergisch Gladbach,

958 Germany, Germany) and digested enzymatically for $20-30$ min at $37^{\circ} \mathrm{C}$ while

959 continuously shaking in a water bath. Adipocytes and stromal vascular fraction cells

960 were separated by centrifugation at $400 \mathrm{~g}$ at $18^{\circ} \mathrm{C}$ for $5 \mathrm{~min}$. After centrifugation, the

961 adipocytes accumulated as fat layer on the surface of the suspension and the SVF

962 cells were pelleted at the bottom. Adipocytes were carefully transferred to a new vial

963 an washed twice in PEB buffer (BSA 0.5\% w/v, 2 mM EDTA, PBS) and then quick 
964 frozen in liquid nitrogen. The SVF cells were washed twice in PEB buffer and then snap

965 frozen as pellet. Blood was collected in EDTA-treated tubes (Sarstedt, Nümbrecht,

966 Germany) and plasma generated by centrifugation at $17000 \mathrm{~g}$ for $10 \mathrm{~min}$ at $4^{\circ} \mathrm{C}$,

967 followed by snap freezing in liquid nitrogen. All samples were stored at $-80^{\circ} \mathrm{C}$ until

968 being used for metabolomic analyses.

969

970 Metabolite Extraction

971 Samples were aliquoted and, if necessary disintegrated with a ball mill (Qiagen,

972 TissueLyser II). Each sample was extracted in $1000 \mu \mathrm{l}$ of pre-cooled $\left(-20^{\circ} \mathrm{C}\right)$ extraction

973 buffer (UPLC-grade actonitrile:methanol:water [40:40:20 v/v]), which was spiked with

$97450 \mathrm{ng} / \mathrm{ml}$ of uridine d2 (Santa Cruz Biotechnology, sc-213138) and UDP-a-D-13C

975 glucose (Cambridge Isotope Laboratories, CLM-10513) as internal standards. The

976 sample was immediately vortexed until the plasma was homogenously suspended and

977 samples were incubated for $30 \mathrm{~min}$ on an orbital mixer at $4^{\circ} \mathrm{C}$ and $1500 \mathrm{rpm}$, before

978 sonicating them for $10 \mathrm{~min}$ in an ice-cooled bath-type sonicator. After sonication,

979 samples were centrifuged for $10 \mathrm{~min}$ at $21.100 \mathrm{xg}$ and $4^{\circ} \mathrm{C}$ and the cleared supernatant

980 was transferred to fresh $1.5 \mathrm{~mL}$ Eppendorf tubes and concentrated to dryness using a

981 Speed Vac concentrator (www.eppendorf.com). In parallel to the analytical samples,

982 we prepared $50 \mu$ l aliquots of reference samples, which were extracted as described

983 above. These reference samples contained distinct concentrations $(0-5000 \mathrm{ng} / \mathrm{ml})$ of

984 the pure reference compounds uridine (Sigma Aldrich, St. Louis, MO, USA; U3750)

985 and UDP (Sigma Aldrich, St. Louis, MO, USA; 94330).

986

987 Quantitative LC-MS Analysis of Uridine

988 For the mass spectrometric analysis of uridine levels, the concentrated metabolite 989 pellet was re-suspended in $100 \mu$ of UPLC grade water. Five $\mu$ l of the cleared 990 supernatant of the re-suspended samples was analyzed on an UPLC (Acquity iClass, 991 Waters $)$, using a HSST3 $(100 \times 1.0 \mathrm{~mm}$ column with $1.8 \mu \mathrm{m}$ particle size, Waters $)$ 992 connected to a Xevo TQ-S (Waters) triple quadrupole mass spectrometer. The flow 993 rate was set to $100 \mu \mathrm{l} / \mathrm{min}$ of buffer $\mathrm{A}(0.1 \%$ formic acid in UPLC grade water) and 994 buffer B (0.1\% formic acid in UPLC-grade acetonitrile) using the following gradient: 0 9951 min 99-90\% A; 1-5 $\min 90-50 \%$ A; 5-6.5 min 50-1\%A; 6.6-7.5 min 1\% A; 7.5-7.7 min $9961-99 \% \mathrm{~A}$. The column was re-equilibrated at $99 \% \mathrm{~A}$ for additional $3.3 \mathrm{~min}$. The eluting 997 metabolites were detected in positive ion mode using MRM (multi reaction monitoring) 
998 applying the following settings: capillary voltage $2.0 \mathrm{kV}$, desolvation temperature $550^{\circ} \mathrm{C}$,

999 desolvation gas flow rate $800 \mathrm{l} / \mathrm{h}$, cone gas flow $150 \mathrm{ml} / \mathrm{min}$, collision cell gas flow 0.15

$1000 \mathrm{ml} / \mathrm{min}$. The following MRM transitions were used for relative compound quantification

1001 of uridine and uridine $d 2$, respectively: $\mathrm{m} / \mathrm{z}$ precursor mass $\left(\mathrm{M}+\mathrm{H}^{+}\right) 245 / 247$, fragment

1002 mass $\left(\mathrm{M}+\mathrm{H}^{+}\right) \mathrm{m} / \mathrm{z}$ 113/115 using a cone voltage of 26/6 $\mathrm{V}$ and a collision energy of

1003 16/10 V. For confirmation purpose of the analyzed compound an additional transition

1004 of the precursor mass $\left(\mathrm{M}+\mathrm{H}^{+}\right) \mathrm{m} / \mathrm{z}$ 245/247, fragment mass $\left(\mathrm{M}+\mathrm{H}^{+}\right) \mathrm{m} / \mathrm{z}$ 133/98 using

1005 a cone voltage of $26 / 6 \mathrm{~V}$ and a collision energy of $16 / 32 \mathrm{~V}$ was analyzed.

1006

\section{Anion-Exchange Chromatography-Mass Spectrometry (AEC-MS) Analysis of 1008 UDP}

1009 Parallel to the analysis of uridine an aqueous aliquot of the re-suspended metabolite

1010 pellet was analyzed using a Dionex ion chromatography system (ICS 5000+, Thermo

1011 Fisher Scientific, Waltham, MA, USA) connected to a QE-HF mass spectrometer

1012 (Thermo Fisher Scientific, Waltham, MA, USA). The applied protocol was adopted from

1013 [2]. In brief: $5 \mu \mathrm{l}$ of the metabolite extract were injected in push partial loop mode using

1014 an overfill factor of 3 , onto a Dionex lonPac AS11-HC column $(2 \mathrm{~mm} \times 250 \mathrm{~mm}, 4 \mu \mathrm{m}$

1015 particle size, Thermo Fisher Scientific, Waltham, MA, USA) equipped with a Dionex

1016 IonPac AG11-HC guard column $(2 \mathrm{~mm} \times 50 \mathrm{~mm}, 4 \mu \mathrm{m}$, Thermo Fisher Scientific,

1017 Waltham, MA, USA). The column temperature was held at $30^{\circ} \mathrm{C}$, while the auto

1018 sampler was set to $6^{\circ} \mathrm{C}$. The metabolites were separated using a potassium hydroxide

1019 gradient, which was generated by an eluent generator using a potassium hydroxide

1020 cartridge that was supplied with deionized water. The AEC flow rate was set to 380

$1021 \mu \mathrm{l} / \mathrm{min}$, applying the following gradient. 0-8 min, 30-35 mM KOH; 8-12 min, 35-100 mM

$1022 \mathrm{KOH}$; 12-15 min, $100 \mathrm{mM} \mathrm{KOH}, 15-15.1 \mathrm{~min}, 100-30 \mathrm{mM} \mathrm{KOH}$. The column was re-

1023 equilibrated at $30 \mathrm{mM} \mathrm{KOH}$ for $3.9 \mathrm{~min}$. The eluting metabolites were detected in

1024 negative ion mode using a MRM method on a Waters TQ triple quadrupole mass

1025 spectrometer (Waters). The MS settings for the analysis were: capillary voltage 2.75

$1026 \mathrm{kV}$, desolvation temperature $550^{\circ} \mathrm{C}$, desolvation gas flow $800 \mathrm{l} / \mathrm{h}$. The following MRM

1027 transitions were used for relative compound quantification of UDP and UDP-13C-GIc,

1028 respectively: $\mathrm{m} / \mathrm{z}$ precursor mass $\left(\mathrm{M}-\mathrm{H}^{+}\right)$403/571, fragment mass $\left(\mathrm{M}-\mathrm{H}^{+}\right) \mathrm{m} / \mathrm{z}$ 159/323

1029 using a cone voltage of $34 / 38 \mathrm{~V}$ and a collision energy of 26/24 V. For confirmation

1030 purpose of the analyzed compound an additional fragment mass $\left(\mathrm{M}-\mathrm{H}^{+}\right) \mathrm{m} / \mathrm{z}$ 111/96

1031 using a cone voltage of $34 / 38 \mathrm{~V}$ and a collision energy of $20 / 34$ was analyzed. 
1032 Data analysis and peak integration was performed using the TargetLynx Software

1033 (Waters). Both uridine and UDP was quantified using an independently generated

1034 calibration curve of the pure reference compound.

1035

\section{Statistical Analyses}

1037 Depending on their genotype, littermate mice were allocated into experimental groups 1038 (P2Y6 $6^{f / f l}$ vs P2Y6 $6^{\Delta N c r 1}$ or P2Y6 $6^{f / f l}$ vs P2Y6 $\left.{ }^{\Delta L y s M}\right)$. For investigations of UDP and uridine 1039 levels, two independent cohorts of C57BI6 mice were randomly assigned to HFD or 1040 CD feeding. The numbers of mice, representing the biological replicate, are included 1041 in the figure legends. Where multiple trials of the same experiment were conducted 1042 (technical replicates), such as for GPCR (duplicates), BMDM culture 1043 (duplicates/triplicates) and immunostainings (duplicates/triplicates), the results of 1044 these technical replicates were averaged and finally plotted as a single replicate.

1045 Statistical significance was tested using Graph Pad Prism 9.0.2 (GraphPad Software 1046 Inc., San Diego, CA, USA) and defined as significant, if $p \leq 0.05$. Statistical tests that 1047 were applied and resulting significance levels are indicated on figures and figure 1048 legends.

\section{Data Availability}

1051 Raw RNASeq data have been deposited in the NCBI Gene Expression Omnibus under 1052 accession code GSE175591. 


\section{Acknowledgements}

1067 We thank all co-workers who supported this work at our institution, with special thanks

1068 to P. Scholl, N. Spenrath, C. Schäfer, K. Marohl, J. Noe and J. Frère for excellent tech-

1069 nical assistance. We thank Dr. T. Wunderlich and Dr. C. Brandt for fruitful scientific

1070 discussions and Dr. H. Broenneke, Dr. U. Lichtenberg, Dr. K. Schoefisch and Dr. R.

1071 Braun for skillful administrative help.

1072

1073

\section{Competing interests}

1074 The authors declare no competing interests.

1075 
1078 Arner and Rydén, 2015. Fatty acids, obesity and insulin resistance. Obesity Facts, 8:147-155. https://doi.org/10.1159/000381224.

1080

1081 Ayala JE, Bracy DP, Malabanan C, James FD, Ansari T, Fueger PT, McGuinness OP, 1082 Wasserman DH. Hyperinsulinemic-euglycemic clamps in conscious, unrestrained mice. 1083 J Vis Exp. 2011 Nov 16;(57):3188. doi: 10.3791/3188. PMID: 22126863; PMCID: 1084 PMC3308587.

1085

1086 Bakker AB, Wu J, Phillips JH, Lanier LL. NK cell activation: distinct stimulatory 1087 pathways counterbalancing inhibitory signals. Hum Immunol. 2000 Jan;61(1):18-27. 1088 doi: 10.1016/s0198-8859(99)00160-3. PMID: 10658974.

1089

1090 Bar I, Guns PJ, Metallo J, Cammarata D, Wilkin F, Boeynams JM, Bult H, Robaye B. 1091 Knockout mice reveal a role for P2Y6 receptor in macrophages, endothelial cells, and 1092 vascular smooth muscle cells. Mol Pharmacol. 2008 Sep;74(3):777-84. doi: 1093 10.1124/mol.108.046904. Epub 2008 Jun 3. PMID: 18523137.

Berg JM, Tymoczko JL, Stryer L. Biochemistry. 5th edition. New York: W H Freeman; https://www.ncbi.nlm.nih.gov/books/NBK21190/. Accessed May 2021.

1098

Borges L, Kubin M, Kuhlman T. LIR9, an immunoglobulin-superfamily-activating receptor, is expressed as a transmembrane and as a secreted molecule. Blood. 2003 Feb 15;101(4):1484-6. doi: 10.1182/blood-2002-05-1432. Epub 2002 Sep 26. PMID: 12393390.

1103

1104 Castoldi A, Naffah de Souza C, Câmara NO, Moraes-Vieira PM. The Macrophage 1105 Switch in Obesity Development. Front Immunol. 2016 Jan 5;6:637. doi: 10.3389/fimmu.2015.00637. PMID: 26779183; PMCID: PMC4700258.

Chen J, Zhao Y, Liu Y. The role of nucleotides and purinergic signaling in apoptotic cell clearance - implications for chronic inflammatory diseases. Front Immunol. 2014 Dec 23;5:656. doi: 10.3389/fimmu.2014.00656. PMID: 25566266; PMCID: PMC4274988.

1113 T. Uridine supplementation exerts anti-inflammatory and anti-fibrotic effects in an 1114 animal model of pulmonary fibrosis. Respir Res. 2015 Sep 15;16(1):105. doi: 10.1186/s12931-015-0264-9. PMID: 26369416; PMCID: PMC4570657. 
1121 Cízek J, Herholz K, Vollmar S, Schrader R, Klein J, Heiss WD. Fast and robust 1122 registration of PET and MR images of human brain. Neuroimage. 2004 May;22(1):4341123 42. doi: 10.1016/j.neuroimage.2004.01.016. PMID: 15110036.

1124

1125 Clausen BE, Burkhardt C, Reith W, Renkawitz R, Förster I. Conditional gene targeting 1126 in macrophages and granulocytes using LysMcre mice. Transgenic Res. 1999 1127 Aug;8(4):265-77. doi: 10.1023/a:1008942828960. PMID: 10621974.

1128

1129 Crinier A, Milpied P, Escalière B, Piperoglou C, Galluso J, Balsamo A, Spinelli L, 1130 Cervera-Marzal I, Ebbo M, Girard-Madoux M, Jaeger S, Bollon E, Hamed S, 1131 Hardwigsen J, Ugolini S, Vély F, Narni-Mancinelli E, Vivier E. High-Dimensional Single1132 Cell Analysis Identifies Organ-Specific Signatures and Conserved NK Cell Subsets in 1133 Humans and Mice. Immunity. 2018 Nov 20;49(5):971-986.e5. doi: 1134 10.1016/j.immuni.2018.09.009. Epub 2018 Nov 6. PMID: 30413361; PMCID: 1135 PMC6269138.

1136

1137 Cursons J, Souza-Fonseca-Guimaraes F, Foroutan M, Anderson A, Hollande F, 1138 Hediyeh-Zadeh S, Behren A, Huntington ND, Davis MJ. A Gene Signature Predicting 1139 Natural Killer Cell Infiltration and Improved Survival in Melanoma Patients. Cancer 1140 Immunol Res. 2019 Jul;7(7):1162-1174. doi: 10.1158/2326-6066.CIR-18-0500. Epub 11412019 May 14. PMID: 31088844.

1142

1143 de Andrade LF, Lu Y, Luoma A, Ito Y, Pan D, Pyrdol JW, Yoon CH, Yuan GC, 1144 Wucherpfennig KW. Discovery of specialized NK cell populations infiltrating human 1145 melanoma metastases. JCI Insight. 2019 Dec 5;4(23):e133103. doi: 1146 10.1172/jci.insight.133103. PMID: 31801909; PMCID: PMC6962021.

1147

1148 de Heredia FP, Gómez-Martínez S, Marcos A. Obesity, inflammation and the immune 1149 system. Proc Nutr Soc. 2012 May;71(2):332-8. doi: 10.1017/S0029665112000092. 1150 Epub 2012 Mar 20. PMID: 22429824.

1151

1152 Deng Y, Wang ZV, Gordillo R, An Y, Zhang C, Liang Q, Yoshino J, Cautivo KM, De 1153 Brabander J, Elmquist JK, Horton JD, Hill JA, Klein S, Scherer PE. An adipo-biliary1154 uridine axis that regulates energy homeostasis. Science. 2017 Mar 1155 17;355(6330):eaaf5375. doi: 10.1126/science.aaf5375. Epub 2017 Mar 16. PMID: 1156 28302796; PMCID: PMC5832364..

1158 Eckelhart E, Warsch W, Zebedin E, Simma O, Stoiber D, Kolbe T, Rülicke T, Mueller 1159 M, Casanova E, Sexl V. A novel Ncr1-Cre mouse reveals the essential role of STAT5 1160 for NK-cell survival and development. Blood. 2011 Feb 3;117(5):1565-73. doi: 1161 10.1182/blood-2010-06-291633. Epub 2010 Dec 2. PMID: 21127177. 1164 of hepatic uridine phosphorylase activity and plasma concentration of uridine in mice. 
1165 Biochem Pharmacol. 1990 Dec 1;40(11):2479-85. doi: 10.1016/0006-2952(90)90089-

1166

1167

1168 Elliott MR, Chekeni FB, Trampont PC, Lazarowski ER, Kadl A, Walk SF, Park D, 1169 1170

4. PMID: 2148479. Woodson RI, Ostankovich M, Sharma P, Lysiak JJ, Harden TK, Leitinger N, Ravichandran KS. Nucleotides released by apoptotic cells act as a find-me signal to promote phagocytic clearance. Nature. 2009 Sep 10;461(7261):282-6. doi: 10.1038/nature08296. PMID: 19741708; PMCID: PMC2851546.

Enerbäck S, Semb H, Tavernier J, Bjursell G, Olivecrona T. Tissue-specific regulation of guinea pig lipoprotein lipase; effects of nutritional state and of tumor necrosis factor on mRNA levels in adipose tissue, heart and liver. Gene. 1988 Apr 15;64(1):97-106. doi: 10.1016/0378-1119(88)90484-2. PMID: 3396878.

Erick TK, Brossay L. Phenotype and functions of conventional and non-conventional NK cells. Curr Opin Immunol. 2016 Feb;38:67-74. doi: 10.1016/j.coi.2015.11.007. Epub 2015 Dec 17. PMID: 26706497; PMCID: PMC4715908.

Evaldsson C, Rydén I, Uppugunduri S. Anti-inflammatory effects of exogenous uridine in an animal model of lung inflammation. Int Immunopharmacol. 2007 Aug;7(8):102532. doi: 10.1016/j.intimp.2007.03.008. Epub 2007 Apr 24. PMID: 17570319.

Ewels P., Hammarén R., Peltzer A., Moreno D., Garcia M., rfenouil; marchoeppner; Senthilkumar Panneerselvam; Sven F.; jun-wan; Johannes Alneberg; aanil; Sofia Haglund; Paolo Di Tommaso; Anders Jemt; KochTobi; Lavanya Veeravalli; Colin Davenport; Rad Suchecki; Max Natthawut Adulyanukosol; Francesco; Denis OMeally; Chuan Wang, 2019. nf-core/rnaseq: nf-core/rnaseq version 1.4 "Gray Crocus Dachshund" (version 1.4). Zenodo. https://doi.org/10.5281/zenodo.3490660.

Filipovic I, Sönnerborg I, Strunz B, Friberg D, Cornillet M, Hertwig L, Ivarsson MA, Björkström NK. 29-Color Flow Cytometry: Unraveling Human Liver NK Cell Repertoire Diversity. Front Immunol. 2019 Nov 19;10:2692. doi: 10.3389/fimmu.2019.02692. PMID: 31798596; PMCID: PMC6878906.

Fruchart-Najib J, Baugé E, Niculescu LS, Pham T, Thomas B, Rommens C, Majd Z, Brewer B, Pennacchio LA, Fruchart JC. Mechanism of triglyceride lowering in mice expressing human apolipoprotein A5. Biochem Biophys Res Commun. 2004 Jun 25;319(2):397-404. doi: 10.1016/j.bbrc.2004.05.003. PMID: 15178420.

Genoux A, Dehondt H, Helleboid-Chapman A, Duhem C, Hum DW, Martin G, Pennacchio LA, Staels B, Fruchart-Najib J, Fruchart JC. Transcriptional regulation of apolipoprotein A5 gene expression by the nuclear receptor RORalpha. Arterioscler Thromb Vasc Biol. 2005 Jun;25(6):1186-92. doi: 10.1161/01.ATV.0000163841.85333.83. Epub 2005 Mar 24. PMID: 15790933. 
1210 Gerrick KY, Gerrick ER, Gupta A, Wheelan SJ, Yegnasubramanian S, Jaffee EM.

1211 Transcriptional profiling identifies novel regulators of macrophage polarization. PLoS

1212 One. 2018 Dec 7;13(12):e0208602. doi: 10.1371/journal.pone.0208602. PMID:

1213 30532146; PMCID: PMC6286176.

1214

1215 Giannattasio G, Ohta S, Boyce JR, Xing W, Balestrieri B, Boyce JA. The purinergic G 1216 protein-coupled receptor 6 inhibits effector $T$ cell activation in allergic pulmonary 1217 inflammation. J Immunol. 2011 Aug 1;187(3):1486-95. doi: 10.4049/jimmunol.1003669.

1218 Epub 2011 Jul 1. PMID: $21724990 ;$ PMCID: PMC3140636.

1219

1220 Girart MV, Fuertes MB, Domaica CI, Rossi LE, Zwirner NW. Engagement of TLR3, 1221 TLR7, and NKG2D regulate IFN-gamma secretion but not NKG2D-mediated 1222 cytotoxicity by human NK cells stimulated with suboptimal doses of IL-12. J Immunol. 12232007 Sep 15;179(6):3472-9. doi: 10.4049/jimmunol.179.6.3472. PMID: 17804388.

1224

1225 Gorini S, Callegari G, Romagnoli G, Mammi C, Mavilio D, Rosano G, Fini M, Di Virgilio 1226 F, Gulinelli S, Falzoni S, Cavani A, Ferrari D, la Sala A. ATP secreted by endothelial 1227 cells blocks $\mathrm{CX}_{3} \mathrm{CL}$ 1-elicited natural killer cell chemotaxis and cytotoxicity via P2Y 11 1228 receptor activation. Blood. 2010 Nov 25;116(22):4492-500. doi: 10.1182/blood-200912-260828. Epub 2010 Jul 28. PMID: 20668227.

1230

1231 Guh DP, Zhang W, Bansback N, Amarsi Z, Birmingham CL, Anis AH. The incidence of 1232 co-morbidities related to obesity and overweight: a systematic review and meta1233 analysis. BMC Public Health. 2009 Mar 25;9:88. doi: 10.1186/1471-2458-9-88. PMID: 1234 19320986; PMCID: PMC2667420.

1235

1236 Hamada T, Mizuta E, Yanagihara K, Kaetsu Y, Sugihara S, Sonoyama K, Yamamoto Y, 1237 Kato M, Igawa O, Shigemasa C, Inokuchi T, Yamamoto T, Shimada T, Ohtahara A, 1238 Ninomiya $\mathrm{H}$, Hisatome I. Plasma levels of uridine correlate with blood pressure and 1239 indicators of myogenic purine degradation and insulin resistance in hypertensive 1240 patients. Circ J. 2007 Mar;71(3):354-6. doi: 10.1253/circj.71.354. PMID: 17322634.

1241

1242 Hashemi E, Malarkannan S. Tissue-Resident NK Cells: Development, Maturation, and

1243 Clinical Relevance. Cancers (Basel). 2020 Jun 12;12(6):1553. doi: 1244 10.3390/cancers12061553. PMID: 32545516; PMCID: PMC7352973.

1245

1246 Haugaard ES, Frantz KB, Haugaard N. Effect of uridine on cellular UTP and glycogen 1247 synthesis in skeletal muscle: stimulation of UTP formation by insulin. Proc Natl Acad 1248 Sci U S A. 1977 Jun;74(6):2339-42. doi: 10.1073/pnas.74.6.2339. PMID: 267929; 1249 PMCID: PMC432166.

1250

1251 Idzko M, Panther E, Sorichter S, Herouy Y, Berod L, Geissler M, Mockenhaupt M, 1252 Elsner P, Girolomoni G, Norgauer J. Characterization of the biological activities of 1253 uridine diphosphate in human dendritic cells: Influence on chemotaxis and CXCL8 
1254 release. J Cell Physiol. 2004 Nov;201(2):286-93. doi: 10.1002/jcp.20070. PMID: 125515334663.

1256

1257 Idzko M, Ferrari D, Eltzschig HK. Nucleotide signalling during inflammation. Nature.

12582014 May 15;509(7500):310-7. doi: 10.1038/nature13085. PMID: 24828189; PMCID:

1259 PMC4222675.

1260

1261 Jablonski KA, Amici SA, Webb LM, Ruiz-Rosado Jde D, Popovich PG, Partida1262 Sanchez S, Guerau-de-Arellano M. Novel Markers to Delineate Murine M1 and M2

1263 Macrophages. PLoS One. 2015 Dec 23;10(12):e0145342. doi: 1264 10.1371/journal.pone.0145342. PMID: 26699615; PMCID: PMC4689374.

1265

1266 Jain S, Pydi SP, Toti KS, Robaye B, Idzko M, Gavrilova O, Wess J, Jacobson KA. Lack 1267 of adipocyte purinergic P2Y6 receptor greatly improves whole body glucose 1268 homeostasis. Proc Natl Acad Sci U S A. 2020 Dec 1;117(48):30763-30774. doi: 1269 10.1073/pnas.2006578117. Epub 2020 Nov 16. PMID: 33199639; PMCID: 1270 PMC7720204.

1272 Jensen MD, Nielsen S. Insulin dose response analysis of free fatty acid kinetics. 1273 Metabolism. 2007 Jan;56(1):68-76. doi: 10.1016/j.metabol.2006.08.022. PMID:

127417161228.

1275

1276 Jewett A, Gan XH, Lebow LT, Bonavida B. Differential secretion of TNF-alpha and IFN1277 gamma by human peripheral blood-derived NK subsets and association with functional 1278 maturation. J Clin Immunol. 1996 Jan;16(1):46-54. doi: 10.1007/BF01540972. PMID: 12798926285.

1280

1281 Kaur G, Trowsdale J, Fugger L. Natural killer cells and their receptors in multiple 1282 sclerosis. Brain. 2013 Sep;136(Pt 9):2657-76. doi: 10.1093/brain/aws159. Epub 2012 1283 Jun 25. PMID: 22734127; PMCID: PMC3754456.

1284

1285 Kim SG, Gao ZG, Soltysiak KA, Chang TS, Brodie C, Jacobson KA. P2Y6 nucleotide 1286 receptor activates $\mathrm{PKC}$ to protect $1321 \mathrm{~N} 1$ astrocytoma cells against tumor necrosis 1287 factor-induced apoptosis. Cell Mol Neurobiol. 2003 Jun;23(3):401-18. doi: 1288 10.1023/a:1023696806609. PMID: 12825835; PMCID: PMC3140713.

1290 Kim B, Jeong HK, Kim JH, Lee SY, Jou I, Joe EH. Uridine 5'-diphosphate induces 1291 chemokine expression in microglia and astrocytes through activation of the P2Y6 1292 receptor. J Immunol. 2011 Mar 15;186(6):3701-9. doi: 10.4049/jimmunol.1000212. 1293 Epub 2011 Feb 11. PMID: 21317391.

1294

1295 Kumar V, Bouameur JE, Bär J, Rice RH, Hornig-Do HT, Roop DR, Schwarz N, 1296 Brodesser S, Thiering S, Leube RE, Wiesner RJ, Vijayaraj P, Brazel CB, Heller S, 1297 Binder H, Löffler-Wirth H, Seibel P, Magin TM. A keratin scaffold regulates epidermal 1298 barrier formation, mitochondrial lipid composition, and activity. J Cell Biol. 2015 Dec 
1299 7;211(5):1057-75. doi: 10.1083/jcb.201404147. Erratum in: J Cell Biol. 2016 Mar 1300 28;212(7):877. PMID: 26644517; PMCID: PMC4674273.

1301

1302 Könner AC, Janoschek R, Plum L, Jordan SD, Rother E, Ma X, Xu C, Enriori P, Hampel

1303 B, Barsh GS, Kahn CR, Cowley MA, Ashcroft FM, Brüning JC. Insulin action in AgRP1304 expressing neurons is required for suppression of hepatic glucose production. Cell 1305 Metab. 2007 Jun;5(6):438-49. doi: 10.1016/j.cmet.2007.05.004. PMID: 17550779.

1306

1307 Lam TK, Yoshii H, Haber CA, Bogdanovic E, Lam L, Fantus IG, Giacca A. Free fatty 1308 acid-induced hepatic insulin resistance: a potential role for protein kinase C-delta. Am $1309 \mathrm{~J}$ Physiol Endocrinol Metab. 2002 Oct;283(4):E682-91. doi: 1310 10.1152/ajpendo.00038.2002. PMID: 12217885.

1312 Lam TK, van de Werve G, Giacca A. Free fatty acids increase basal hepatic glucose 1313 production and induce hepatic insulin resistance at different sites. Am J Physiol 1314 Endocrinol Metab. 2003 Feb;284(2):E281-90. doi: 10.1152/ajpendo.00332.2002. 1315 PMID: 12531742.

1316

1317 Lanier LL. Up on the tightrope: natural killer cell activation and inhibition. Nat Immunol.

13182008 May;9(5):495-502. doi: 10.1038/ni1581. PMID: 18425106; PMCID: PMC2669298.

1319

1320 Lattin JE, Schroder K, Su AI, Walker JR, Zhang J, Wiltshire T, Saijo K, Glass CK, Hume 1321 DA, Kellie S, Sweet MJ. Expression analysis of G Protein-Coupled Receptors in mouse 1322 macrophages. Immunome Res. 2008 Apr 29;4:5. doi: 10.1186/1745-7580-4-5. PMID: 1323 18442421; PMCID: PMC2394514.

1324

1325 Lazarowski ER, Sesma JI, Seminario-Vidal L, Kreda SM. Molecular mechanisms of 1326 purine and pyrimidine nucleotide release. Adv Pharmacol. 2011;61:221-61. doi: 10.1016/B978-0-12-385526-8.00008-4. PMID: 21586361.

1328

1329 Le TT, Ziemba A, Urasaki Y, Hayes E, Brotman S, Pizzorno G. Disruption of uridine 1330 homeostasis links liver pyrimidine metabolism to lipid accumulation. J Lipid Res. 2013 1331 Apr;54(4):1044-57. doi: 10.1194/jlr.M034249. Epub 2013 Jan 24. PMID: 23355744; 1332 PMCID: PMC3605981.

1333

1334 Lee BC, Kim MS, Pae M, Yamamoto Y, Eberlé D, Shimada T, Kamei N, Park HS, 1335 Sasorith S, Woo JR, You J, Mosher W, Brady HJ, Shoelson SE, Lee J. Adipose Natural 1336 Killer Cells Regulate Adipose Tissue Macrophages to Promote Insulin Resistance in 1337 Obesity. Cell Metab. 2016 Apr 12;23(4):685-98. doi: 10.1016/j.cmet.2016.03.002. Epub 13382016 Mar 31. PMID: 27050305; PMCID: PMC4833527.

1340 Lewis GF, Vranic M, Harley P, Giacca A. Fatty acids mediate the acute extrahepatic 1341 effects of insulin on hepatic glucose production in humans. Diabetes. 1997 1342 Jul;46(7):1111-9. doi: 10.2337/diab.46.7.1111. PMID: 9200644. 
1344 Li R, Tan B, Yan Y, Ma X, Zhang N, Zhang Z, Liu M, Qian M, Du B. Extracellular UDP 1345 and P2Y6 function as a danger signal to protect mice from vesicular stomatitis virus 1346 infection through an increase in IFN- $\beta$ production. J Immunol. 2014 Nov 1;193(9):45151347 26. doi: 10.4049/jimmunol.1301930. Epub 2014 Sep 26. PMID: 25261483.

1348

1349 Liu G, Xu JN, Liu D, Ding Q, Liu MN, Chen R, Fan M, Zhang Y, Zheng C, Zou DJ, Lyu $1350 \mathrm{~J}$, Zhang WJ. Regulation of plasma lipid homeostasis by hepatic lipoprotein lipase in 1351 adult mice. J Lipid Res. 2016 Jul;57(7):1155-61. doi: 10.1194/jlr.M065011. Epub 2016 1352 May 27. PMID: 27234787; PMCID: PMC4918845.

1353

1354 Love MI, Huber W, Anders S. Moderated estimation of fold change and dispersion for 1355 RNA-seq data with DESeq2. Genome Biol. 2014;15(12):550. doi: 10.1186/s130591356 014-0550-8. PMID: 25516281; PMCID: PMC4302049.

1357

1358 Michelet X, Dyck L, Hogan A, Loftus RM, Duquette D, Wei K, Beyaz S, Tavakkoli A, 1359 Foley C, Donnelly R, O'Farrelly C, Raverdeau M, Vernon A, Pettee W, O'Shea D, 1360 Nikolajczyk BS, Mills KHG, Brenner MB, Finlay D, Lynch L. Metabolic reprogramming 1361 of natural killer cells in obesity limits antitumor responses. Nat Immunol. 2018 1362 Dec;19(12):1330-1340. doi: 10.1038/s41590-018-0251-7. Epub 2018 Nov 12. PMID: 136330420624.

1364

1365 Mitchell A, Rentero C, Endoh Y, Hsu K, Gaus K, Geczy C, McNeil HP, Borges L, Tedla $1366 \mathrm{~N}$. LILRA5 is expressed by synovial tissue macrophages in rheumatoid arthritis, 1367 selectively induces pro-inflammatory cytokines and IL-10 and is regulated by TNF1368 alpha, IL-10 and IFN-gamma. Eur J Immunol. 2008 Dec;38(12):3459-73. doi: 1369 10.1002/eji.200838415. PMID: 19009525.

1370

1371 Murano I, Barbatelli G, Parisani V, Latini C, Muzzonigro G, Castellucci M, Cinti S. Dead 1372 adipocytes, detected as crown-like structures, are prevalent in visceral fat depots of 1373 genetically obese mice. J Lipid Res. 2008 Jul;49(7):1562-8. doi: 10.1194/jlr.M8000191374 JLR200. Epub 2008 Apr 3. PMID: 18390487.

1375

1376 Nielsen TS, Jessen N, Jørgensen JO, Møller N, Lund S. Dissecting adipose tissue 1377 lipolysis: molecular regulation and implications for metabolic disease. J Mol Endocrinol. 13782014 Jun;52(3):R199-222. doi: 10.1530/JME-13-0277. Epub 2014 Feb 27. PMID: 137924577718.

1381 Nowak M, Helleboid-Chapman A, Jakel H, Martin G, Duran-Sandoval D, Staels B, 1382 Rubin EM, Pennacchio LA, Taskinen MR, Fruchart-Najib J, Fruchart JC. Insulin1383 mediated down-regulation of apolipoprotein A5 gene expression through the 1384 phosphatidylinositol 3-kinase pathway: role of upstream stimulatory factor. Mol Cell 1385 Biol. 2005 Feb;25(4):1537-48. doi: 10.1128/MCB.25.4.1537-1548.2005. PMID: 1386 15684402; PMCID: PMC548024. 
1388 Oishi S, Takano R, Tamura S, Tani S, Iwaizumi M, Hamaya Y, Takagaki K, Nagata T, 1389 Seto S, Horii T, Osawa S, Furuta T, Miyajima H, Sugimoto K. M2 polarization of murine 1390 peritoneal macrophages induces regulatory cytokine production and suppresses T-cell 1391 proliferation. Immunology. 2016 Nov;149(3):320-328. doi: 10.1111/imm.12647. Epub 13922016 Aug 9. PMID: 27421990; PMCID: PMC5046056.

1393

1394 Paeger L, Karakasilioti I, Altmüller J, Frommolt P, Brüning J, Kloppenburg P. 1395 Antagonistic modulation of NPY/AgRP and POMC neurons in the arcuate nucleus by 1396 noradrenalin. Elife. 2017 Jun 20;6:e25770. doi: 10.7554/eLife.25770. PMID: 28632132; 1397 PMCID: PMC5478265.

1398

1399 Pardo J, Balkow S, Anel A, Simon MM. Granzymes are essential for natural killer cell1400 mediated and perf-facilitated tumor control. Eur J Immunol. 2002 Oct;32(10):2881-7. 1401 doi: 10.1002/1521-4141(2002010)32:10<2881::AID-IMMU2881>3.0.CO;2-K. PMID: 140212355441.

1403

1404 Patro R, Duggal G, Love MI, Irizarry RA, Kingsford C. Salmon provides fast and bias1405 aware quantification of transcript expression. Nat Methods. 2017 Apr;14(4):417-419. 1406 doi: 10.1038/nmeth.4197. Epub 2017 Mar 6. PMID: 28263959; PMCID: PMC5600148.

1408 Peng H, Jiang X, Chen Y, Sojka DK, Wei H, Gao X, Sun R, Yokoyama WM, Tian Z. 1409 Liver-resident NK cells confer adaptive immunity in skin-contact inflammation. J Clin 1410 Invest. 2013 Apr;123(4):1444-56. doi: 10.1172/JCI66381. Epub 2013 Mar 25. PMID: 1411 23524967; PMCID: PMC3613925.

1413 Peng H, Tian Z. Diversity of tissue-resident NK cells. Semin Immunol. 2017 Jun;31:31414 10. doi: 10.1016/j.smim.2017.07.006. Epub 2017 Aug 9. PMID: 28802693.

1415

1416 Ramos P, Martín-Hidalgo A, Herrera E. Insulin-induced up-regulation of lipoprotein 1417 lipase messenger ribonucleic acid and activity in mammary gland. Endocrinology. 1999 1418 Mar;140(3):1089-93. doi: 10.1210/endo.140.3.6565. PMID: 10067830.

1419

1420 Rebrin K, Steil GM, Mittelman SD, Bergman RN. Causal linkage between insulin 1421 suppression of lipolysis and suppression of liver glucose output in dogs. J Clin Invest. 14221996 Aug 1;98(3):741-9. doi: 10.1172/JCI118846. PMID: 8698866; PMCID: 1423 PMC507484.

1425 Schmidt FM, Weschenfelder J, Sander C, Minkwitz J, Thormann J, Chittka T, Mergl R, 1426 Kirkby KC, Faßhauer M, Stumvoll M, Holdt LM, Teupser D, Hegerl U, Himmerich H. 1427 Inflammatory cytokines in general and central obesity and modulating effects of 1428 physical activity. PLoS One. 2015 Mar 17;10(3):e0121971. doi: 1429 10.1371/journal.pone.0121971. PMID: 25781614; PMCID: PMC4363366.

1431 Smith SL, Kennedy PR, Stacey KB, Worboys JD, Yarwood A, Seo S, Solloa EH, 1432 Mistretta B, Chatterjee SS, Gunaratne P, Allette K, Wang YC, Smith ML, Sebra R, Mace 
1433 EM, Horowitz A, Thomson W, Martin P, Eyre S, Davis DM. Diversity of peripheral blood 1434 human NK cells identified by single-cell RNA sequencing. Blood Adv. 2020 Apr 1435 14;4(7):1388-1406. doi: 10.1182/bloodadvances.2019000699. PMID: 32271902; 1436 PMCID: PMC7160259.

1437

1438 Steculorum SM, Paeger L, Bremser S, Evers N, Hinze Y, Idzko M, Kloppenburg P, 1439 Brüning JC. Hypothalamic UDP Increases in Obesity and Promotes Feeding via P2Y61440 Dependent Activation of AgRP Neurons. Cell. 2015 Sep 10;162(6):1404-17. doi: 1441 10.1016/j.cell.2015.08.032. PMID: 26359991.

1442

1443 Steculorum SM, Timper K, Engström Ruud L, Evers N, Paeger L, Bremser S, 1444 Kloppenburg P, Brüning JC. Inhibition of P2Y6 Signaling in AgRP Neurons Reduces 1445 Food Intake and Improves Systemic Insulin Sensitivity in Obesity. Cell Rep. 2017 Feb 1446 14;18(7):1587-1597. doi: 10.1016/j.celrep.2017.01.047. PMID: 28199831.

1447

1448 Sojka DK, Tian Z, Yokoyama WM. Tissue-resident natural killer cells and their potential 1449 diversity. Semin Immunol. 2014 Apr;26(2):127-31. doi: 10.1016/j.smim.2014.01.010. 1450 Epub 2014 Feb 16. PMID: 24548893; PMCID: PMC4459495.

1451

1452 Sojka DK, Plougastel-Douglas B, Yang L, Pak-Wittel MA, Artyomov MN, Ivanova Y, 1453 Zhong C, Chase JM, Rothman PB, Yu J, Riley JK, Zhu J, Tian Z, Yokoyama WM. 1454 Tissue-resident natural killer (NK) cells are cell lineages distinct from thymic and 1455 conventional splenic NK cells. Elife. 2014 Jan 1;3:e01659. doi: 10.7554/eLife.01659. 1456 PMID: 24714492; PMCID: PMC3975579.

1457

1458 Theurich S, Tsaousidou E, Hanssen R, Lempradl AM, Mauer J, Timper K, Schilbach K, 1459 Folz-Donahue K, Heilinger C, Sexl V, Pospisilik JA, Wunderlich FT, Brüning JC. IL1460 6/Stat3-Dependent Induction of a Distinct, Obesity-Associated NK Cell Subpopulation 1461 Deteriorates Energy and Glucose Homeostasis. Cell Metab. 2017 Jul 5;26(1):1711462 184.e6. doi: 10.1016/j.cmet.2017.05.018. PMID: 28683285.

1463

1464 Turpin-Nolan SM, Hammerschmidt P, Chen W, Jais A, Timper K, Awazawa M, 1465 Brodesser S, Brüning JC. CerS1-Derived C18:0 Ceramide in Skeletal Muscle 1466 Promotes Obesity-Induced Insulin Resistance. Cell Rep. 2019 Jan 2;26(1):1-10.e7. doi: 1467 10.1016/j.celrep.2018.12.031. PMID: 30605666.

1469 Uppugunduri S, Gautam C. Effects of uridine, isomatitol and 4-thiouridine on in vitro 1470 cell adhesion and in vivo effects of 4-thiouridine in a lung inflammation model. Int 1471 Immunopharmacol. 2004 Sep;4(9):1241-8. doi: 10.1016/j.intimp.2004.04.016. PMID: 147215251120.

1474 Urasaki Y, Pizzorno G, Le TT. Uridine affects liver protein glycosylation, insulin 1475 signaling, and heme biosynthesis. PLoS One. 2014 Jun 11;9(6):e99728. doi: 1476 10.1371/journal.pone.0099728. PMID: 24918436; PMCID: PMC4053524. 
1478 Urasaki Y, Pizzorno G, Le TT. Chronic Uridine Administration Induces Fatty Liver and 1479 Pre-Diabetic Conditions in Mice. PLoS One. 2016 Jan 20;11(1):e0146994. doi: 1480 10.1371/journal.pone.0146994. PMID: 26789264; PMCID: PMC4720477.

1481

1482 Vivier, E, Tomasello, E, Baratin, M, Walzer T, Ugolini S. Functions of natural killer cells.

1483 Nat Immunol 9, 503-510 (2008). https://doi.org/10.1038/ni1582

1484

1485 Wang R, Jaw JJ, Stutzman NC, Zou Z, Sun PD. Natural killer cell-produced IFN-y and 1486 TNF- $\alpha$ induce target cell cytolysis through up-regulation of ICAM-1. J Leukoc Biol. 2012 1487 Feb;91(2):299-309. doi: 10.1189/jlb.0611308. Epub 2011 Nov 1. PMID: 22045868; 1488 PMCID: PMC3290424.

1489

1490 Weisberg SP, McCann D, Desai M, Rosenbaum M, Leibel RL, Ferrante AW Jr. Obesity 1491 is associated with macrophage accumulation in adipose tissue. J Clin Invest. 2003 1492 Dec;112(12):1796-808. doi: 10.1172/JCI19246. PMID: 14679176; PMCID: 1493 PMC296995.

1494

1495 Wensveen FM, Jelenčić V, Valentić S, Šestan M, Wensveen TT, Theurich S, Glasner 1496 A, Mendrila D, Štimac D, Wunderlich FT, Brüning JC, Mandelboim O, Polić B. NK cells 1497 link obesity-induced adipose stress to inflammation and insulin resistance. Nat 1498 Immunol. 2015 Apr;16(4):376-85. doi: 10.1038/ni.3120. Epub 2015 Mar 2. PMID: 149925729921.

1500

1501 WHO, 2021. https://www.who.int/news-room/fact-sheets/detail/obesity-and1502 overweight. Accessed May 2021.

1503

1504 Xu H, Barnes GT, Yang Q, Tan G, Yang D, Chou CJ, Sole J, Nichols A, Ross JS, 1505 Tartaglia LA, Chen $\mathrm{H}$. Chronic inflammation in fat plays a crucial role in the 1506 development of obesity-related insulin resistance. J Clin Invest. 2003 1507 Dec;112(12):1821-30. doi: 10.1172/JCI19451. PMID: 14679177; PMCID: PMC296998.

1509 Yamamoto T, Moriwaki Y, Takahashi S, Tsutsumi Z, Yamakita J, Higashino K. Effect of 1510 muscular exercise on the concentration of uridine and purine bases in plasma-1511 adenosine triphosphate consumption-induced pyrimidine degradation. Metabolism. 15121997 Nov;46(11):1339-42. doi: 10.1016/s0026-0495(97)90241-9. PMID: 9361696.

1514 Yang C, Siebert JR, Burns R, Gerbec ZJ, Bonacci B, Rymaszewski A, Rau M, Riese 1515 MJ, Rao S, Carlson KS, Routes JM, Verbsky JW, Thakar MS, Malarkannan S. 1516 Heterogeneity of human bone marrow and blood natural killer cells defined by single1517 cell transcriptome. Nat Commun. 2019 Sep 2;10(1):3931. doi: 10.1038/s41467-0191518 11947-7. PMID: 31477722; PMCID: PMC6718415.

1520 Yu G, Wang LG, Han Y, He QY. clusterProfiler: an R package for comparing biological 1521 themes among gene clusters. OMICS. 2012 May;16(5):284-7. doi: 1522 10.1089/omi.2011.0118. Epub 2012 Mar 28. PMID: 22455463; PMCID: PMC3339379. 
1523 Zhang Z, Wang Z, Ren H, Yue M, Huang K, Gu H, Liu M, Du B, Qian M. P2Y(6) agonist 1524 uridine 5'-diphosphate promotes host defense against bacterial infection via monocyte

1525 chemoattractant protein-1-mediated monocytes/macrophages recruitment. J Immunol.

15262011 May 1;186(9):5376-87. doi: 10.4049/jimmunol.1002946. Epub 2011 Mar 28.

1527 PMID: 21444765.

1528

1529 Zhao J, Zhang S, Liu Y, He X, Qu M, Xu G, Wang H, Huang M, Pan J, Liu Z, Li Z, Liu

$1530 \mathrm{~L}$, Zhang Z. Single-cell RNA sequencing reveals the heterogeneity of liver-resident

1531 immune cells in human. Cell Discov. 2020 Apr 28;6(1):22. doi: 10.1038/s41421-020-

1532 0157-z. PMID: 33907176.

1533

1534 Zwirner NW, Domaica Cl. Cytokine regulation of natural killer cell effector functions.

1535 Biofactors. 2010 Jul-Aug;36(4):274-88. doi: 10.1002/biof.107. PMID: 20623510.

1536

1537

1538

1539

1540

1541

1542

1543

1544

1545

1546

1547

1548

1549

1550

1551

1552

1553

1554

1555

1556

1557

1558

1559

1560

1561

1562

1563

1564

1565

1566

1567 
$1570 \mu \mathrm{CT}$

1571 AgRP

1572 AKT

1573 Apoa5

1574 ATP

1575 BAT

1576 BMDM

1577 Ccl3

1578 Ccl4

1579 CD

1580 CLS

1581 cNK

1582 Cxcl16

1583 DAG

1584 DN

1585 ECAR

1586 OCR

1587 ELISA

1588 ERK

1589 FFA

1590 GIR

1591 GTT

1592 HFD

1593 HGP

1594 HOMA-IR

$1595 \mathrm{HSL}$

1596 IFNy

1597 IL

1598 ILC

1599 ITT

1600 Lilra5

1601 Lpl

1602 MAPK

1603 NCD

1604 NK cell

1605 OCR

1606 OXPHOS

1607 P2Y6R

1608 PGAT

1609 pHSL

1610 PI3K

1611 PKC

1612 Plin2
Micro computer tomography

Agouti-related protein

Protein kinase $B$

Apolipoprotein A-V

Adenosine triphosphate

Brown adipose tissue

Bone marrow-derived macrophages

Chemokine (C-C motif) ligand 3

Chemokine (C-C motif) ligand 4

Control Diet

Crown-like structures

conventional NK cells

Chemokine (C-X-C motif) ligand 16

Diacylglycerides

Double negative

Extracellular acidification rate

Oxygen consumption rate

Enzyme-linked immunosorbent assay

Extracellular signal-regulated kinases

Free fatty acids

Glucose infusion rate

Glucose tolerance test

High fat diet

Hepatic glucose production

Homeostasis model assessment- insulin resistance

Hormone-sensitive lipase

Interferon gamma

Interleukin

Innate lymphoid cell

Insulin tolerance test

Leukocyte immunoglobulin like receptor A5

Lipoprotein lipase

Mitogen-activated protein kinase

Normal chow diet

Natural killer cell

Oxygen consumption rate

Oxidative phosphorylation

P2Y purinoreceptor 6

Perigonodal adipose tissue

phosphorylated hormone-sensitive lipase

Phosphoinositide 3-kinase

Protein kinase $\mathrm{C}$

Perilipin 2 


$\begin{array}{lll}1613 & \text { RER } & \text { Respiratory exchange rate } \\ 1614 & \text { Rora } & \text { RAR-related orphan receptor alpha } \\ 1615 & \text { SEM } & \text { Standard error of the mean } \\ 1616 & \text { SKM } & \text { Skeletal muscle } \\ 1617 & \text { SVF } & \text { Stromal vascular fraction } \\ 1618 & \text { TG } & \text { Triglycerides } \\ 1619 & \text { TNFa } & \text { Tumor necrosis factor alpha } \\ 1620 & \text { TPM } & \text { Transcripts per million } \\ 1621 & \text { trNK } & \text { tissue-resident NK cells } \\ 1622 & \text { UDP } & \text { Uridine diphosphate } \\ 1623 & \text { uNK } & \text { uterine NK cells } \\ 1624 & \text { Xcl1 } & \text { Chemokine (C motif) ligand }\end{array}$


A Body weight development

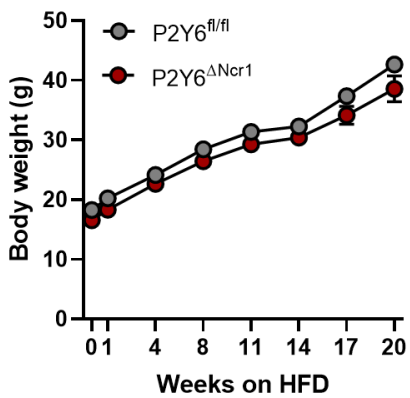

C

Organ weights

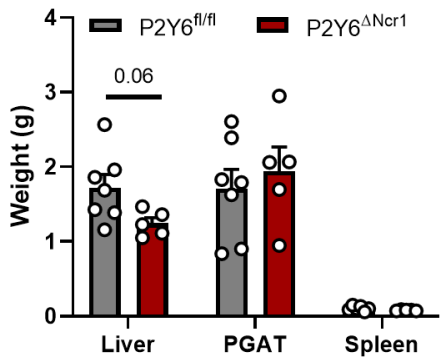

B Body composition

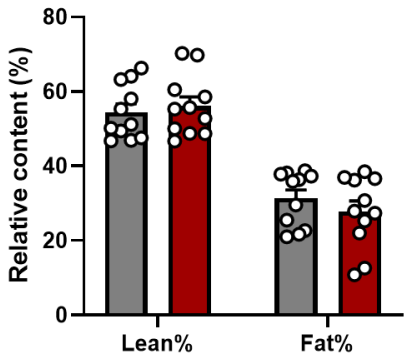

D

Leptin

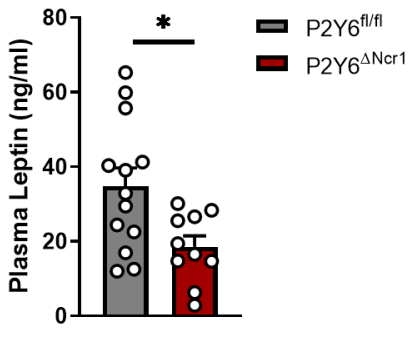


A

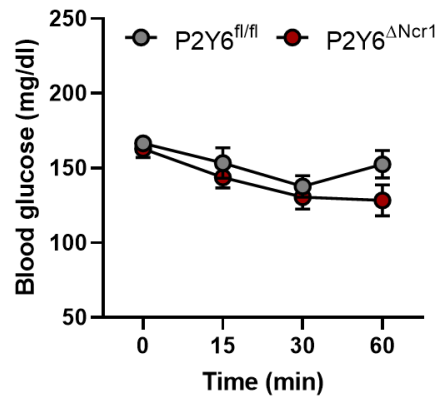

D

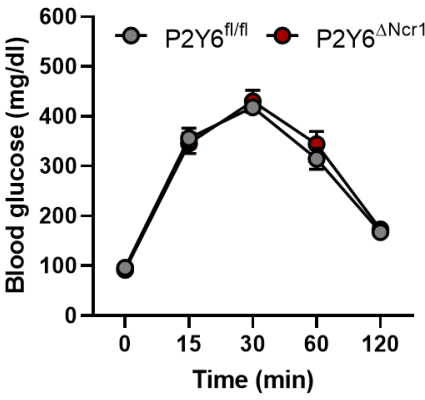

G Fasted blood glucose levels

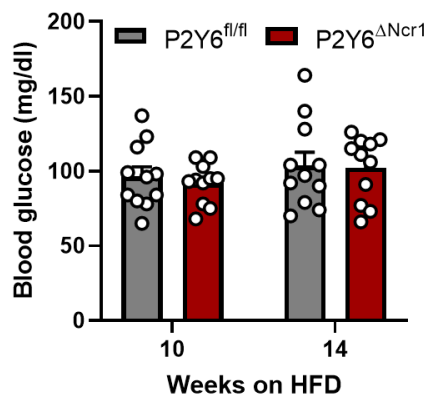

B

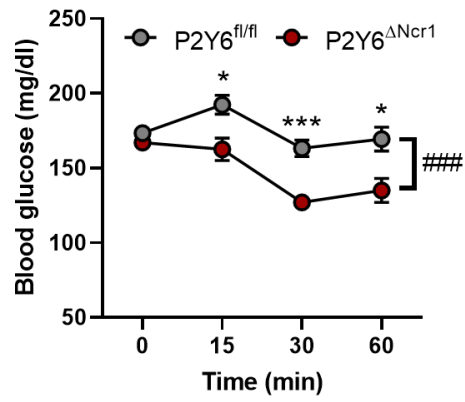

E

GTT 14w HFD

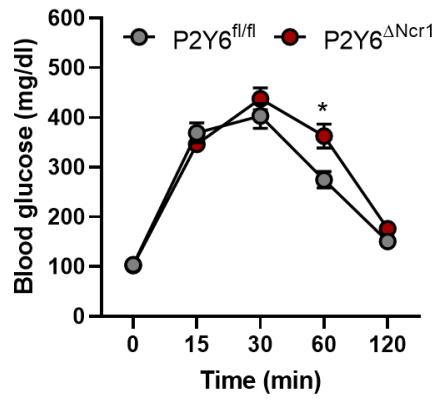

H

Insulin

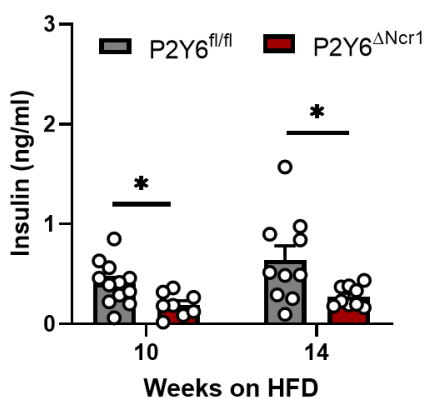

C

ITT 20w HFD

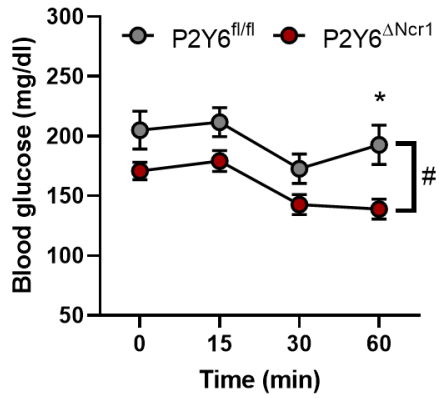

F

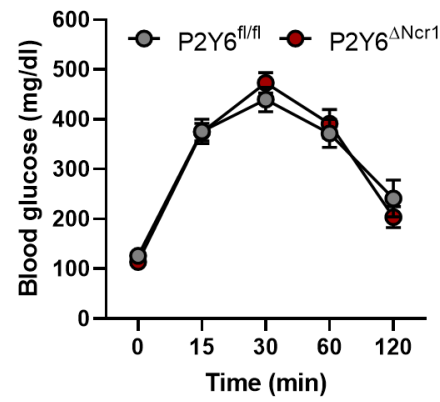

HOMA-IR

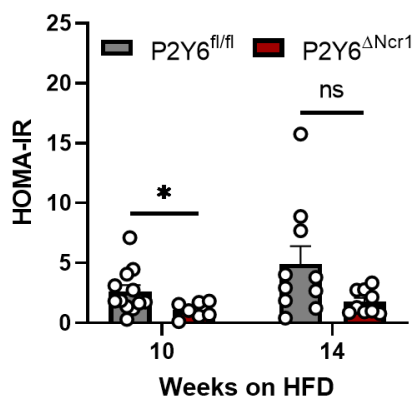


A Glucose infusion rate

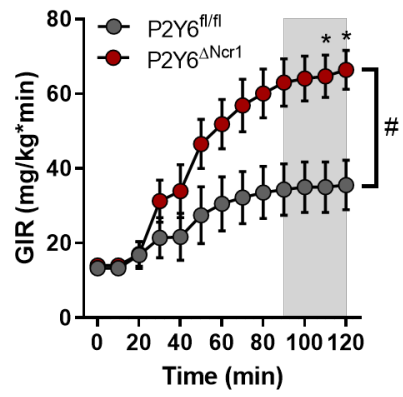

E

FFA Plasma

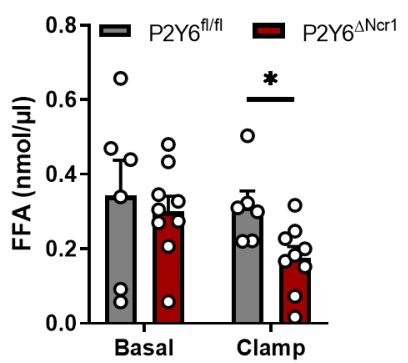

B Blood glucose levels

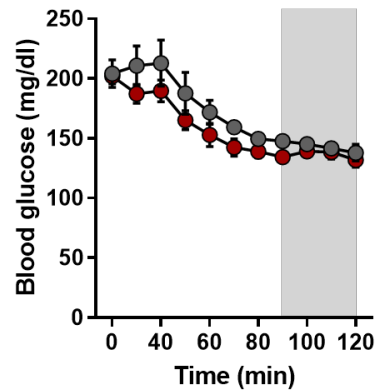

F
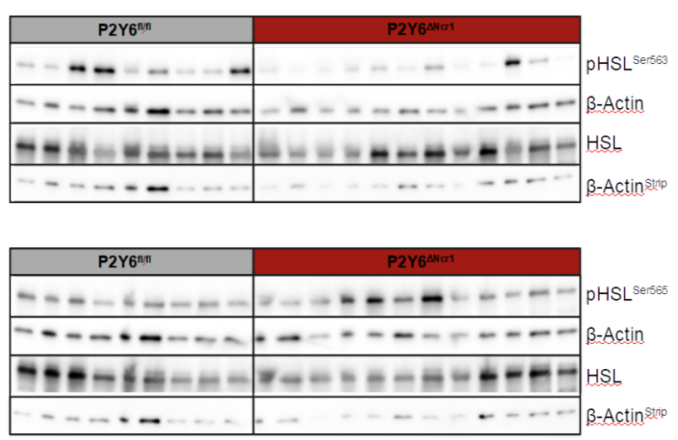

D glucose production

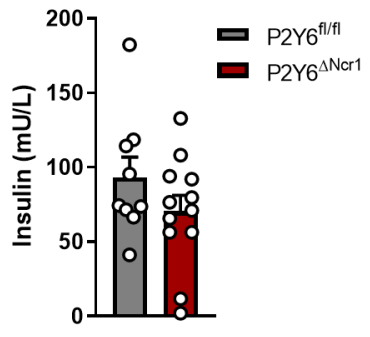

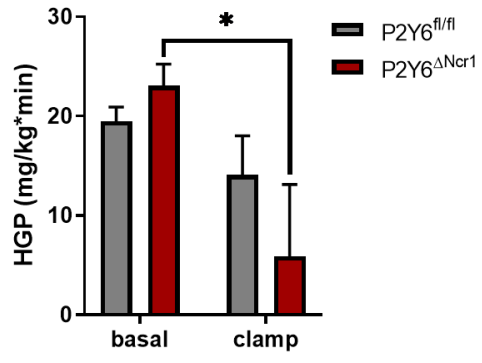

HSL phosphorylation

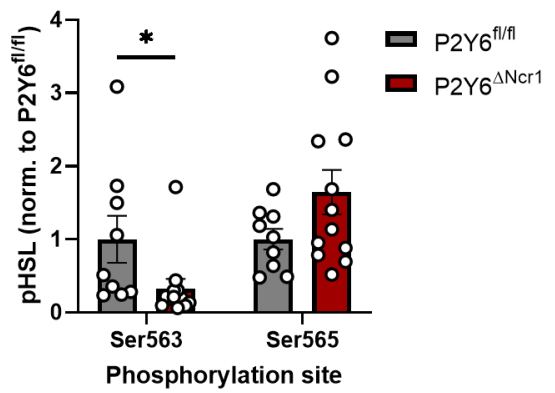

G

Glucose uptake into tissues

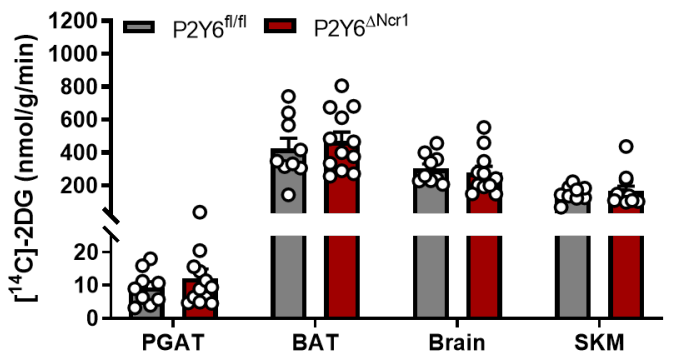




\begin{tabular}{|c|c|}
\hline P2Y6 fl/fil & P2Y6 ${ }^{\Delta \text { Nar1 }}$ \\
\hline$-\cdots-\cdots-\cdots$ & $-\cdots-\cdots-\cdots$ \\
\hline$---\cdots-\cdots$ & $-\cdots-\cdots-\cdots-\cdots$ \\
\hline$-\pi n=-m=-$ & H \\
\hline$\rightarrow-\cdots-\cdots$ & $-\cdots-\cdots-\cdots$ \\
\hline
\end{tabular}

\begin{tabular}{|c|c|}
\hline P2Y6 ${ }^{\text {fl/fII }}$ & P2Y6 ${ }^{\Delta \text { Ner1 }}$ \\
\hline 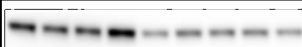 & $--\infty-\cdots-\cdots-\cdots$ \\
\hline$---\infty$ & --- \\
\hline
\end{tabular}

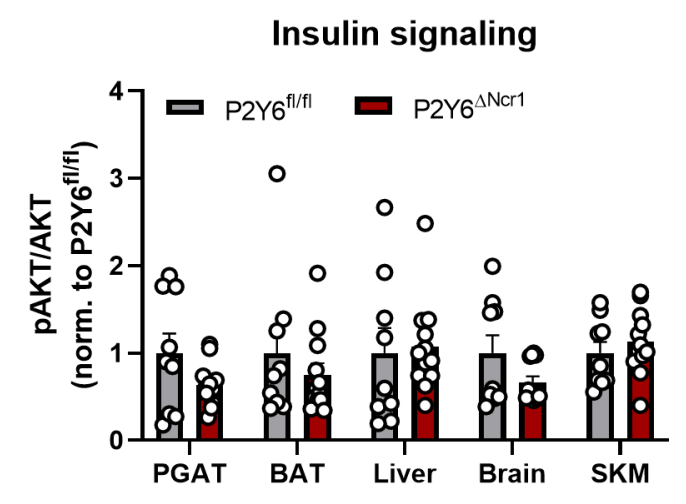

BAT

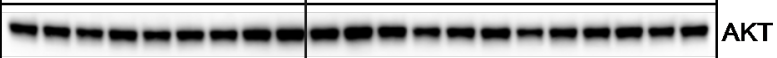

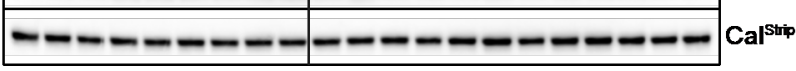

SKM
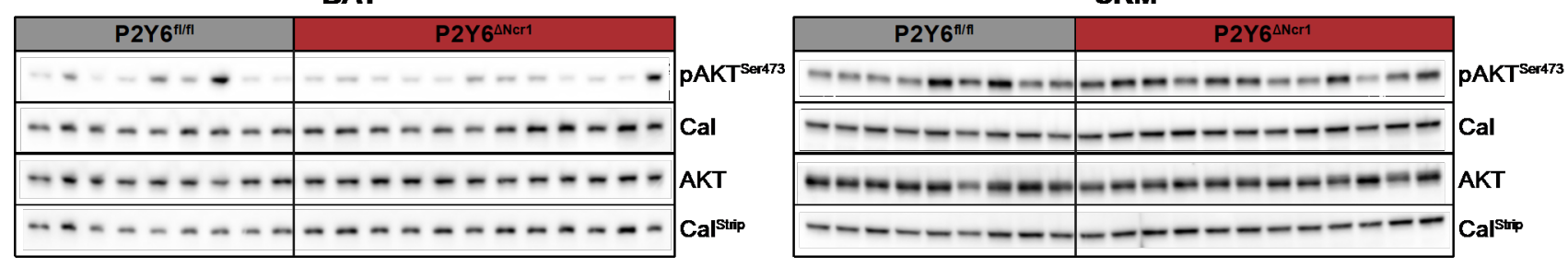

Liver

\begin{tabular}{|c|c|c|}
\hline$\overline{P 2 Y 6} 6^{\text {flffl }}$ & $\mathrm{P} 2 \mathrm{Y} 6^{\Delta \mathrm{Ncr} 1}$ & \\
\hline$-\quad----\cdots$ & $-1-\cdots-\cdots+\cdots$ & pAKT ${ }^{\text {Ser } 473}$ \\
\hline--------- & $--\cdots-\cdots-\cdots---$ & Cal \\
\hline$-----n-\infty$ & 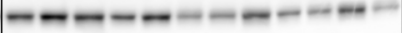 & AKT \\
\hline----- & $-\cdots-\cdots-\cdots$ & Cal ${ }^{\text {stip }}$ \\
\hline
\end{tabular}



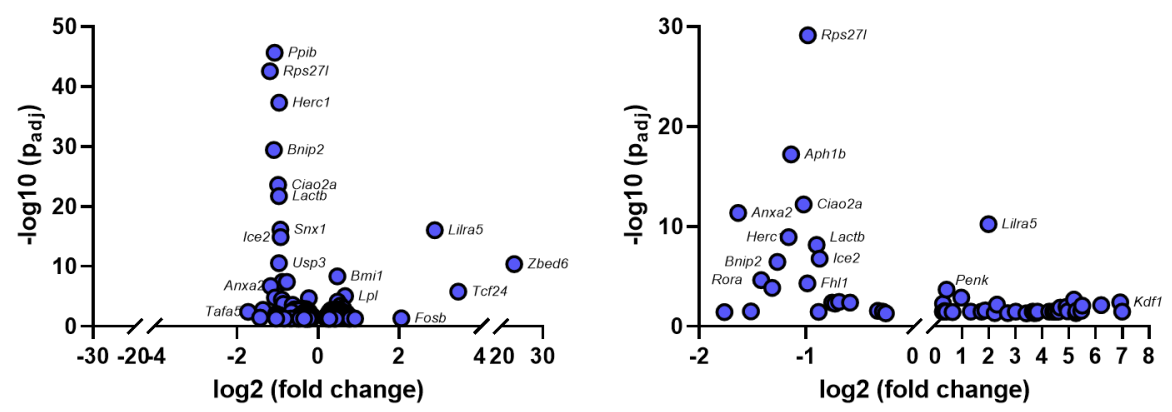

B
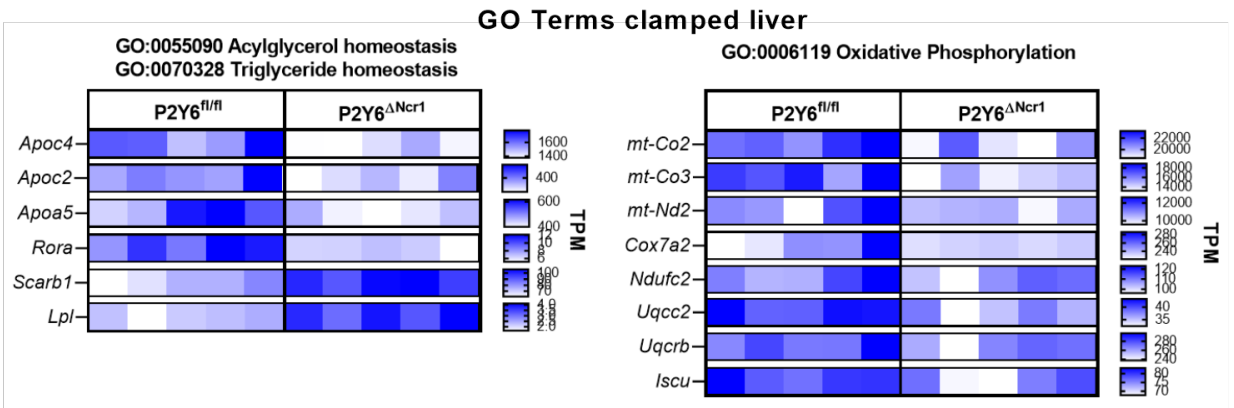

C

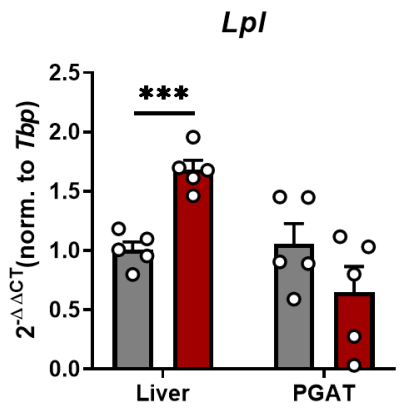

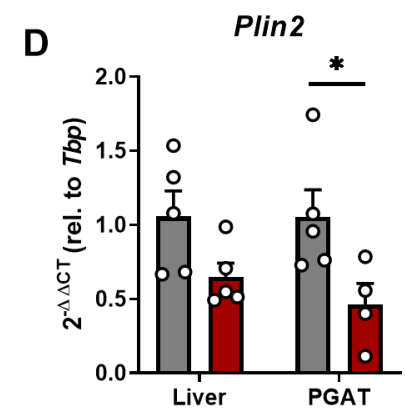
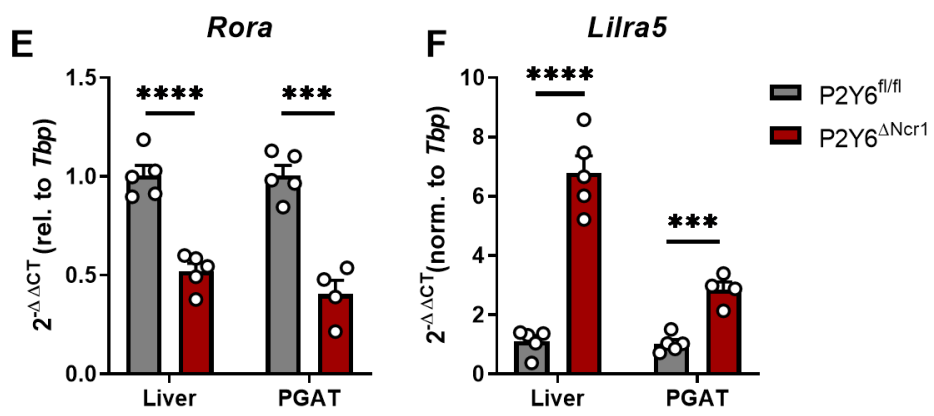

G

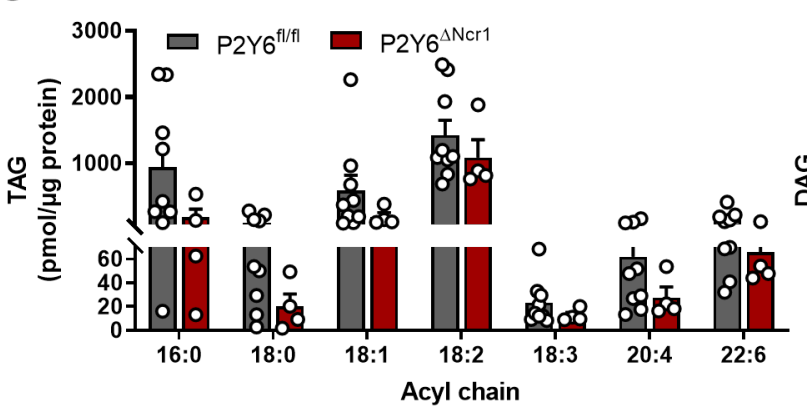

OCR/ECAR basal

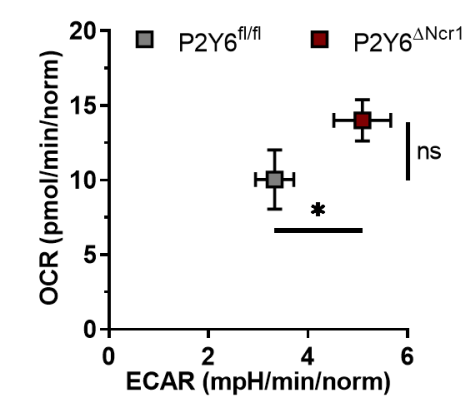

H

Hepatic Diacylglycerides

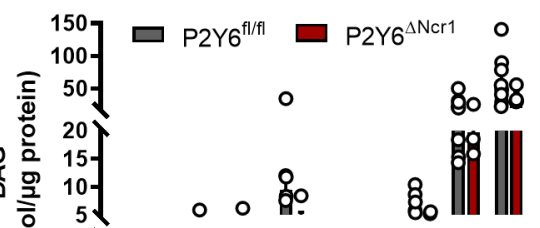

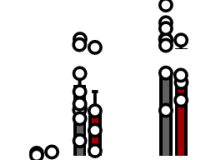

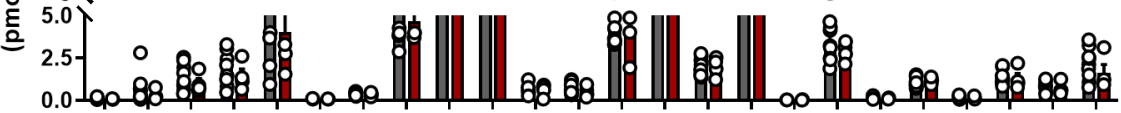

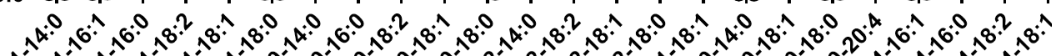

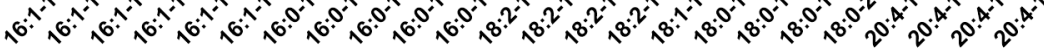
Acyl chain 


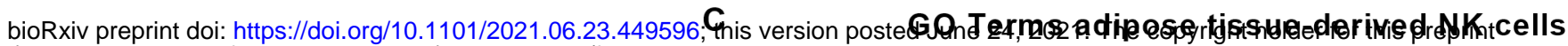

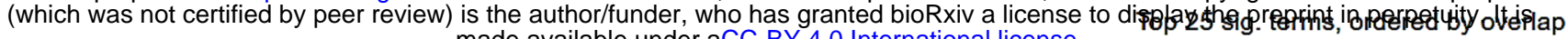

\section{Adipose tissue-derived NK cells}

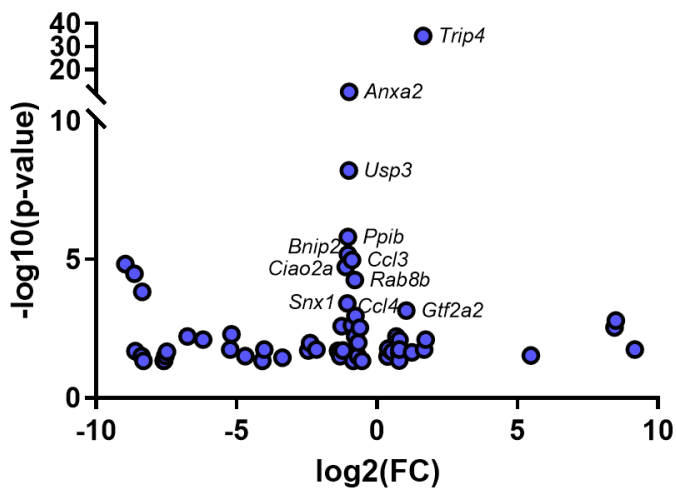

B

\section{Liver-derived NK cells}

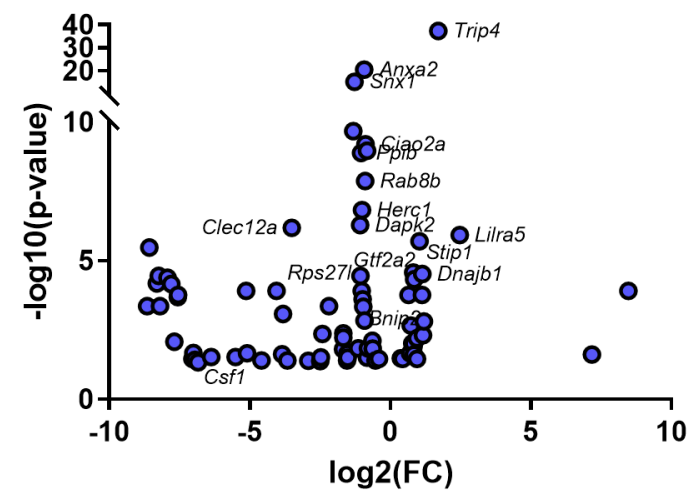

E

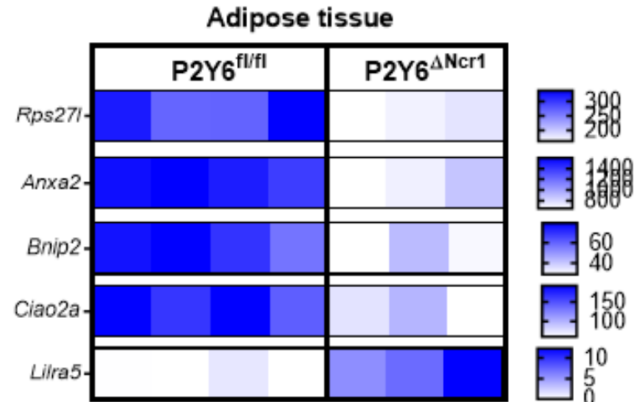

$\mathbf{F}$
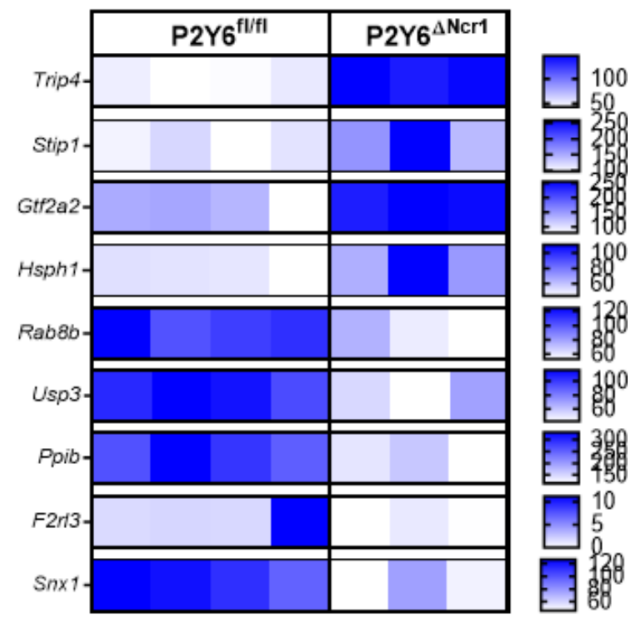

G

GO:0048247 Lymphocyte chemotaxis

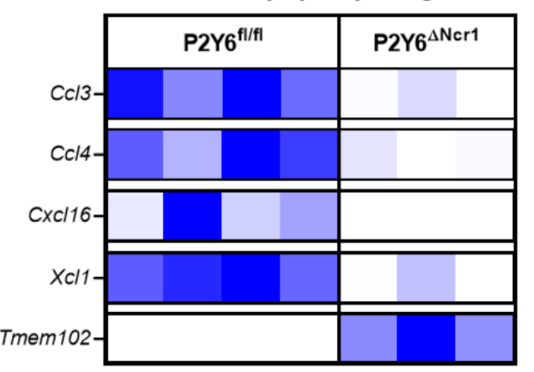

positive regulation of lymphocyte migration

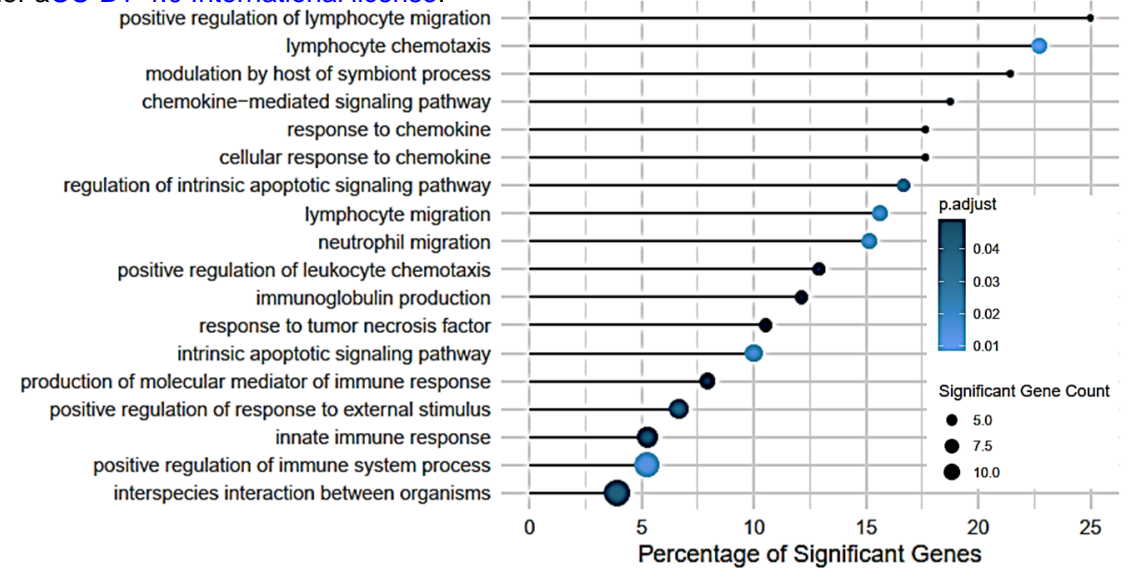

D

GO Terms liver-derived NK cells

Top 25 sig. terms, ordered by overlap

chaperone cofactor-dependent protein refolding
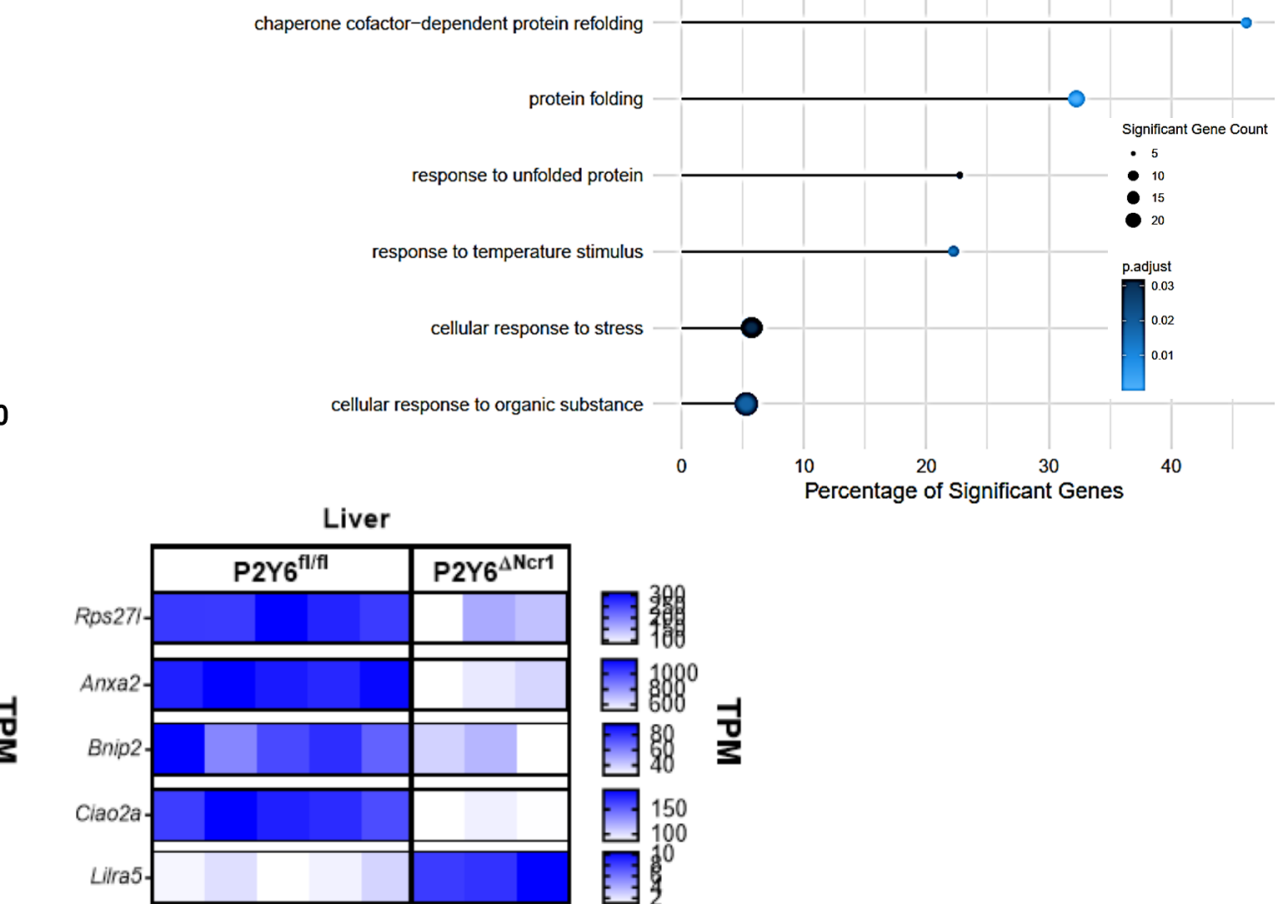

Liver

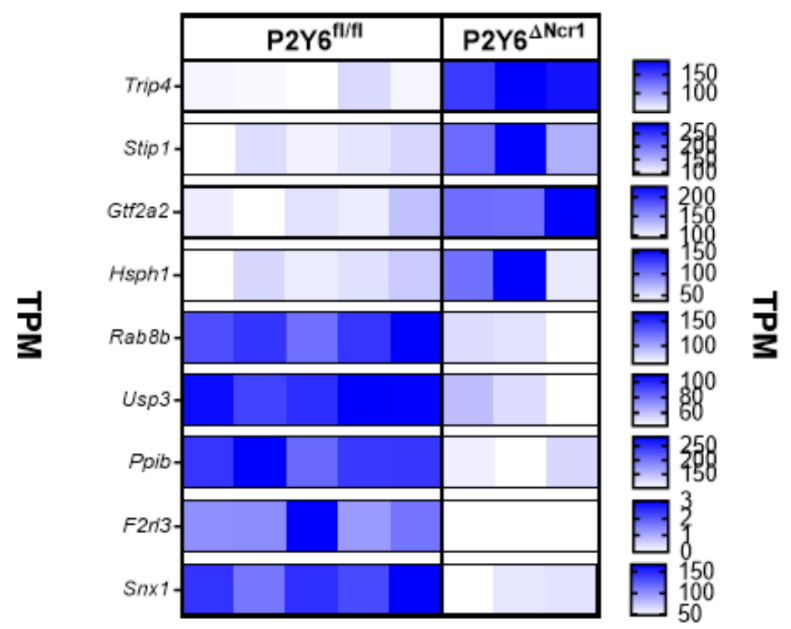


A

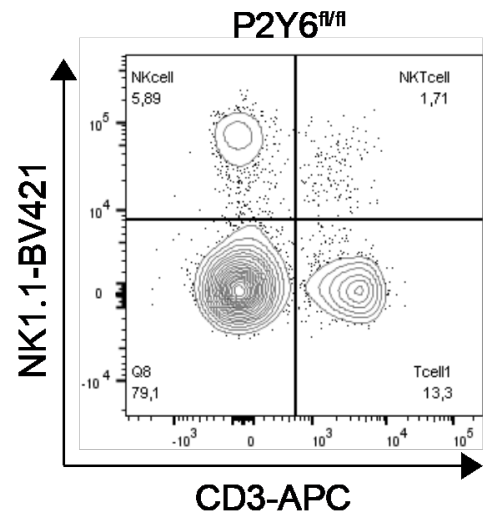

PGAT

Liver
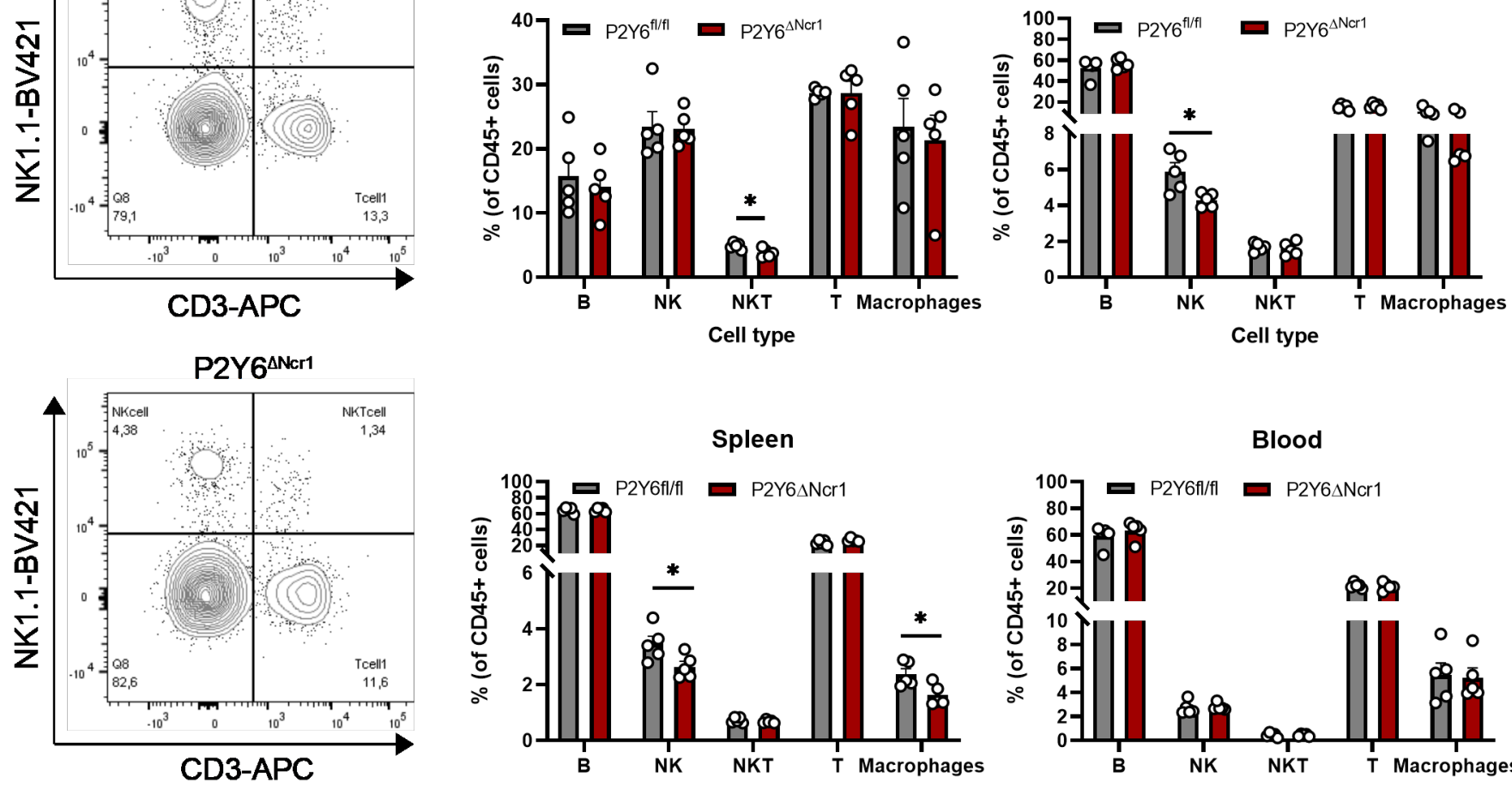

B
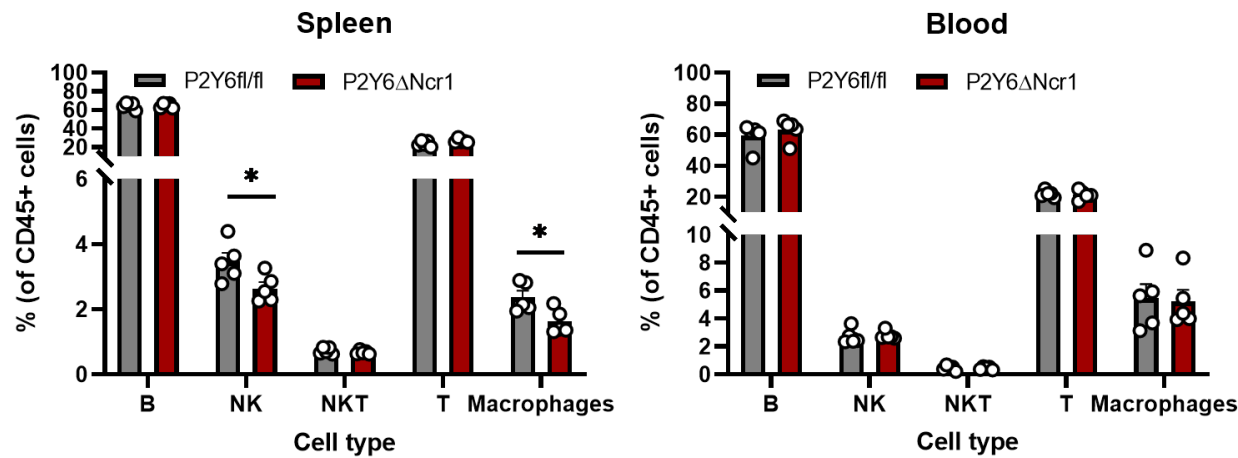

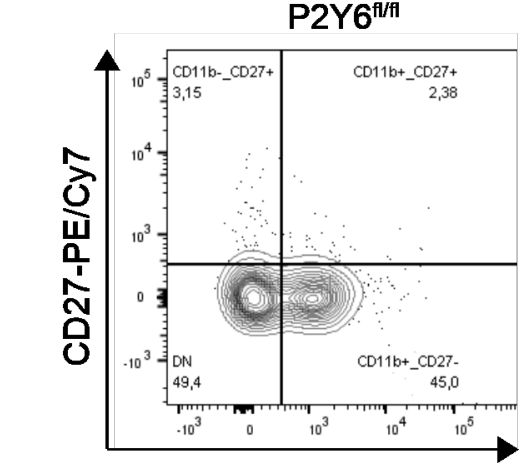

CD11b-PerCP/Cy5

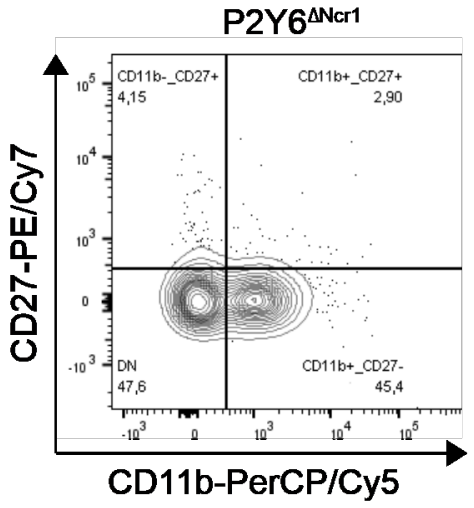

PGAT

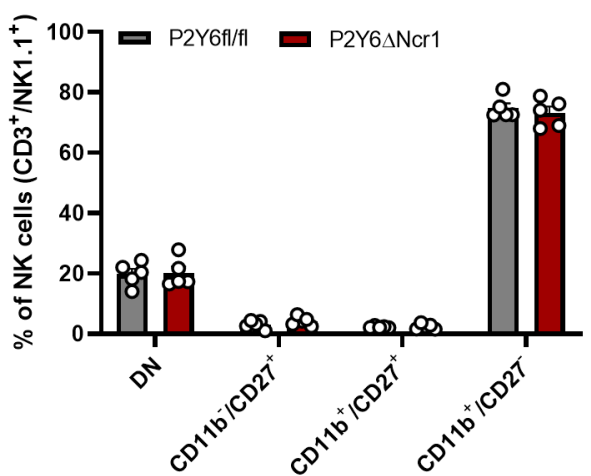

Spleen

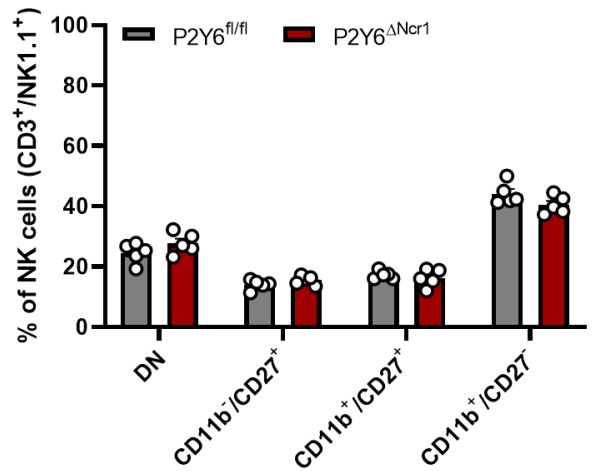

Liver

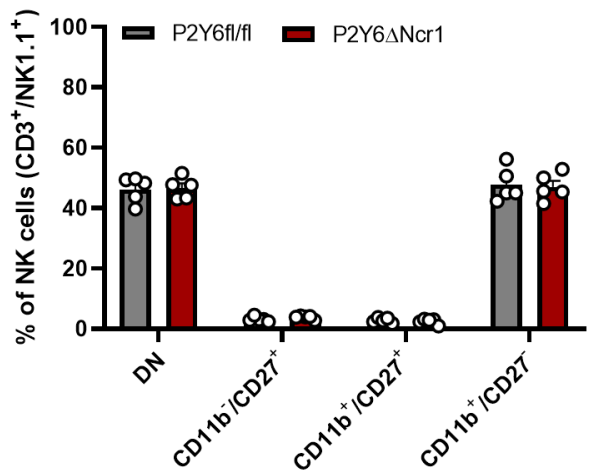

Blood

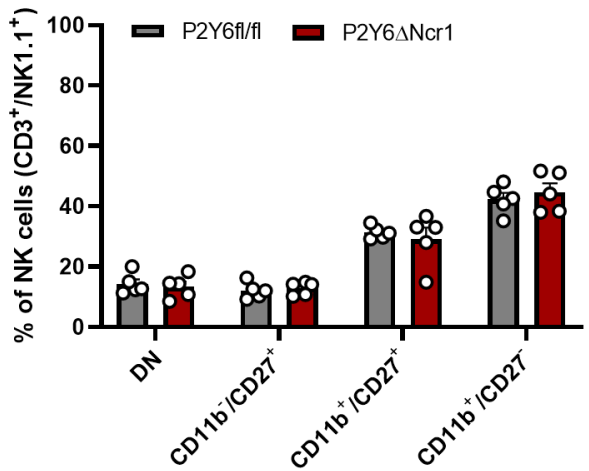


A

Body weight development

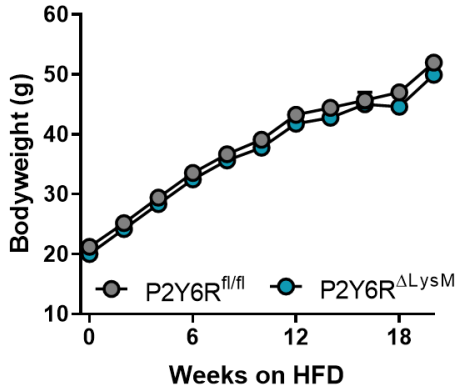

C

ITT 9w HFD

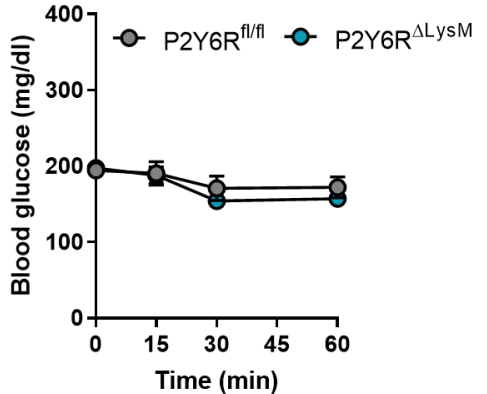

$\mathbf{F}$

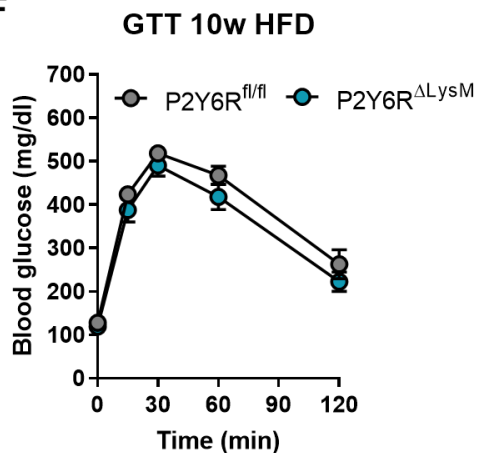

Insulin

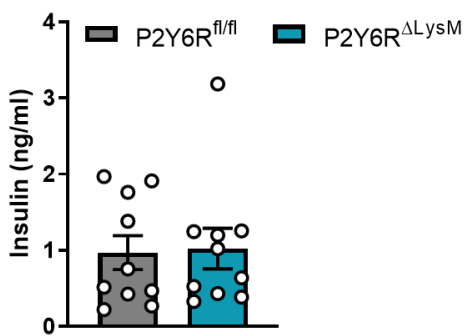

B

Body composition

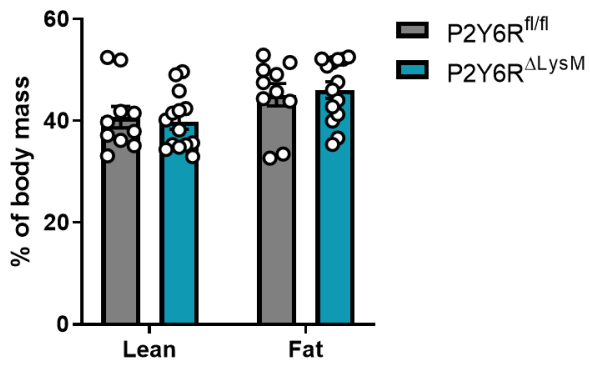

D

ITT 14w HFD

E

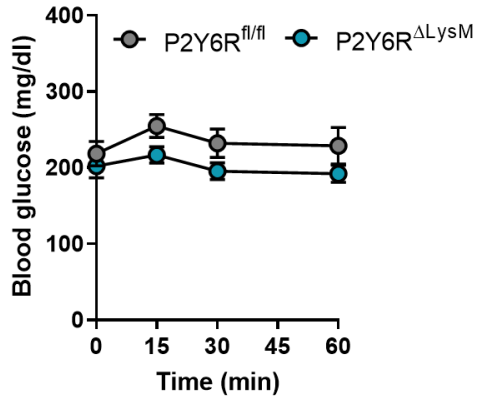

G

GTT 15w HFD

H
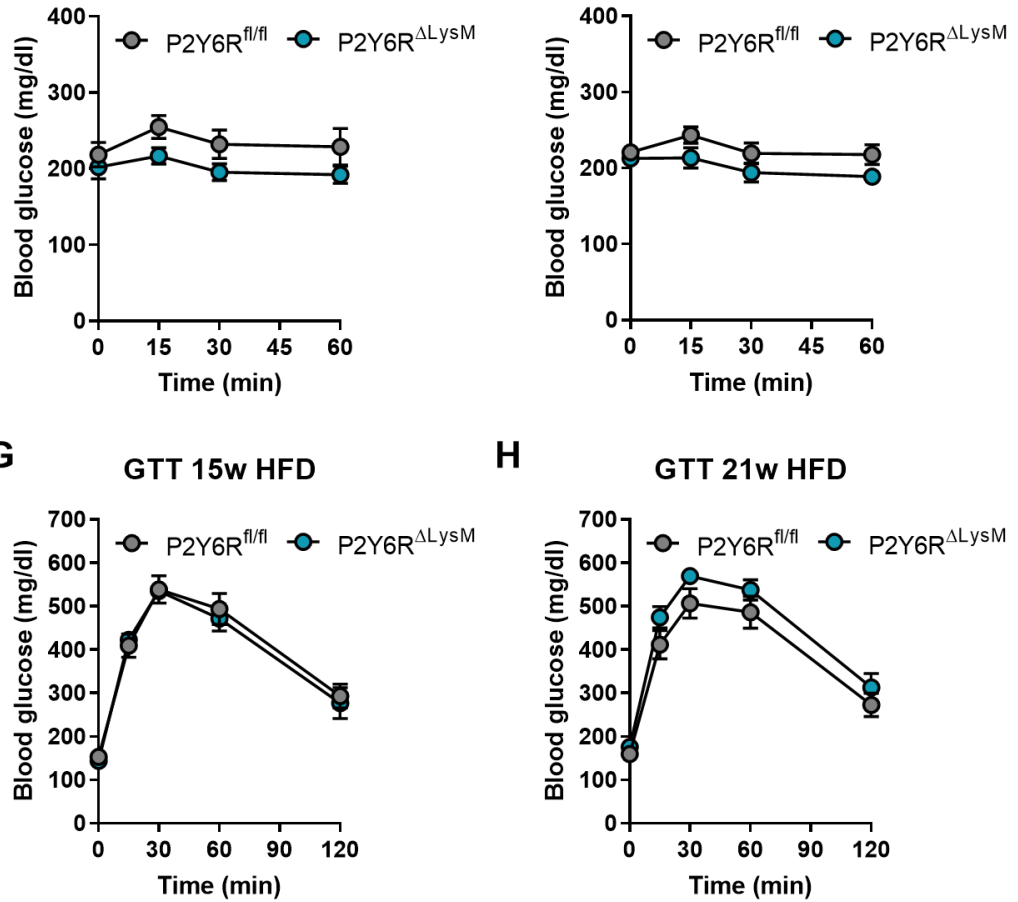

J

Leptin

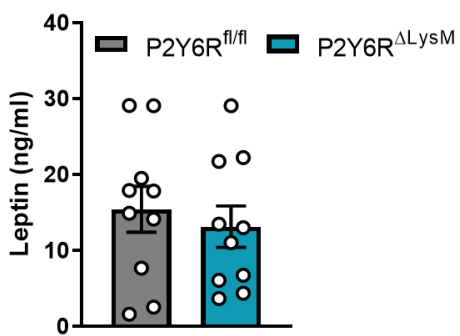

HOMA-IR

GTT 21w HFD

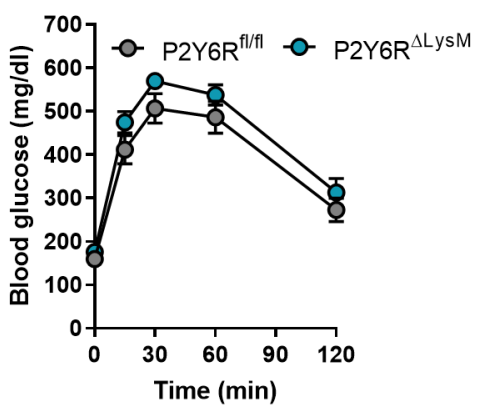

K

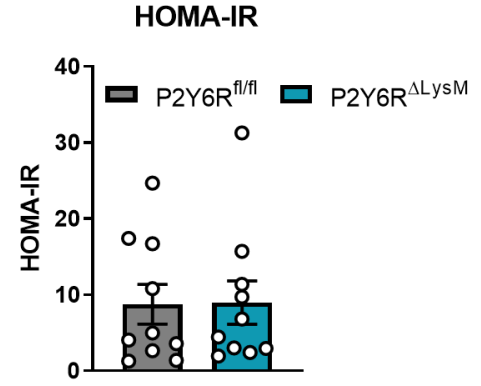


A

Plasma Uridine

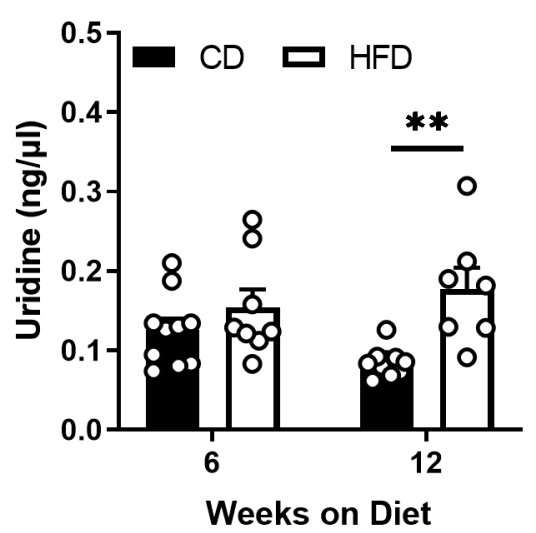

E

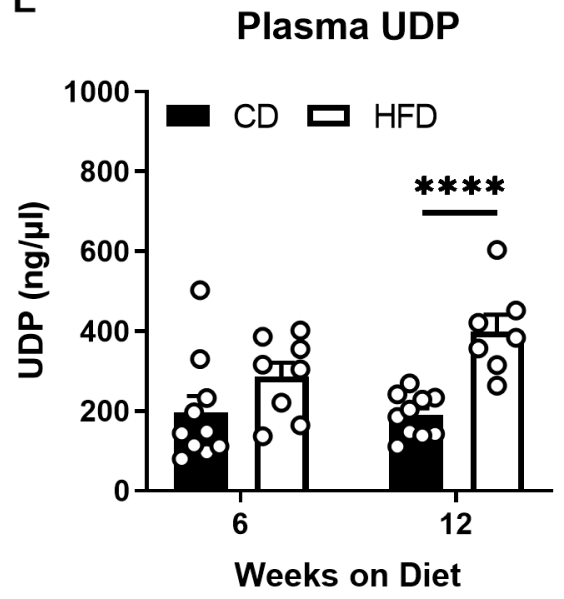

B

Hepatic Uridine

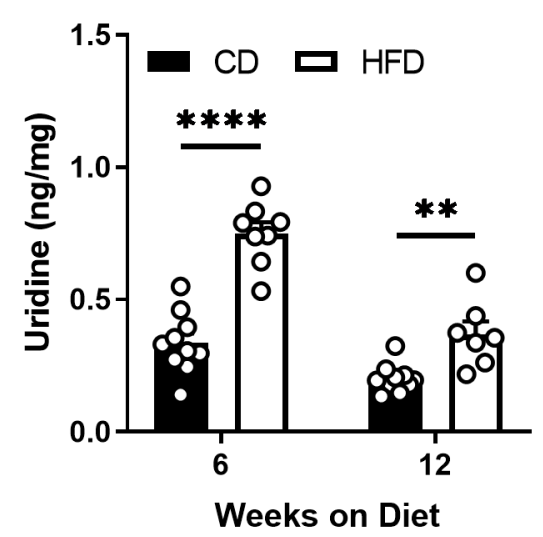

F

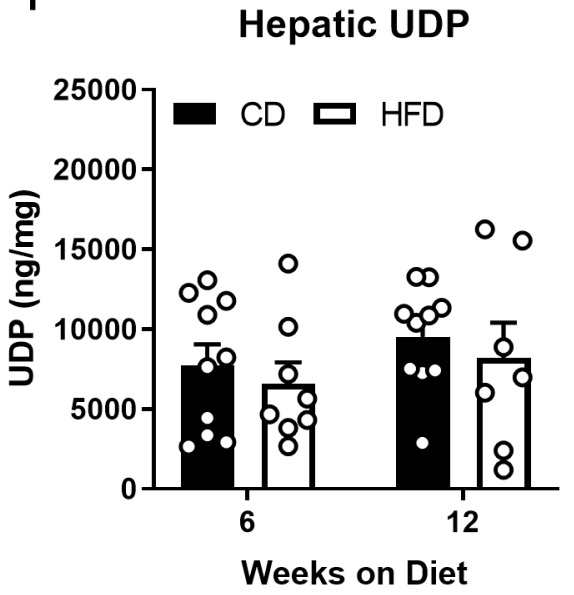

C

SVF Uridine

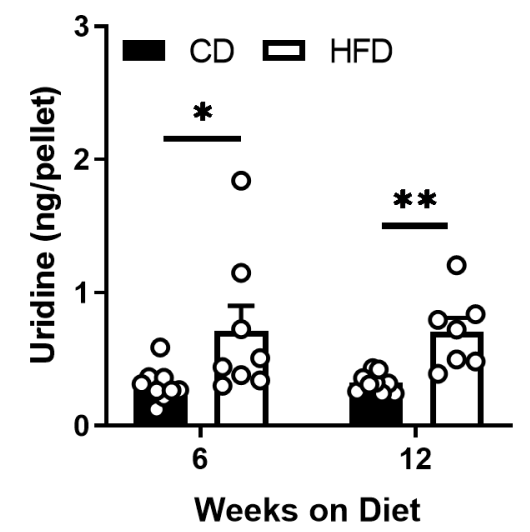

G

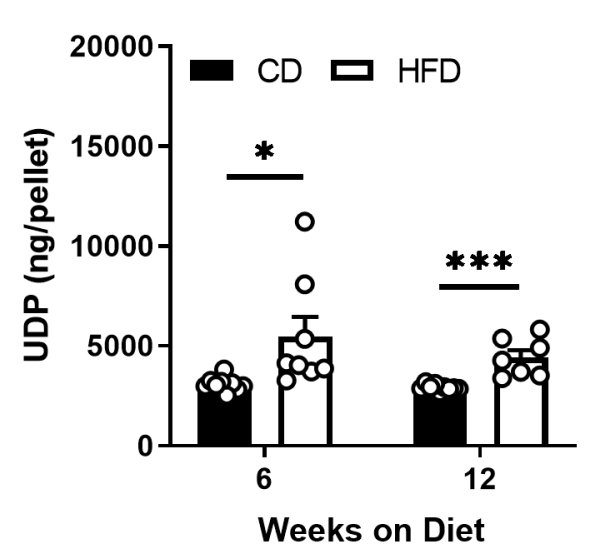

Adipocyte Uridine

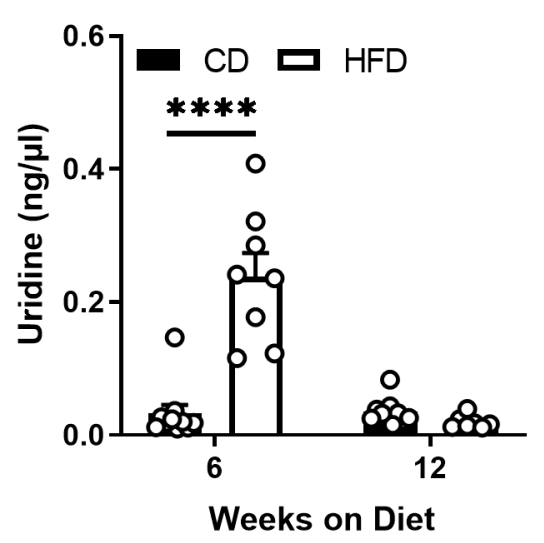

H

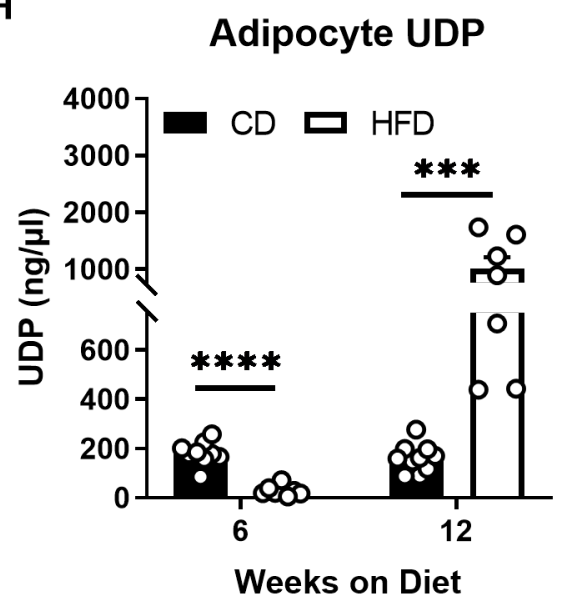


nonNK

P2Y6 ${ }^{\mathrm{f} / 1 \mathrm{fl}}$
nonNK

P2Y6 ${ }^{\Delta \text { Ncr1 }}$
NK

P2Y6 $6^{\text {fl/f }}$
NK

P2Y6 ${ }^{\Delta \mathrm{Ncr} 1}$

Ncr1-Cre $<650$ bp (WT) $\leftarrow 450$ bp (TG) $\triangle \mathrm{P} 2 \mathrm{Y} 6$

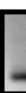


A Body weight development

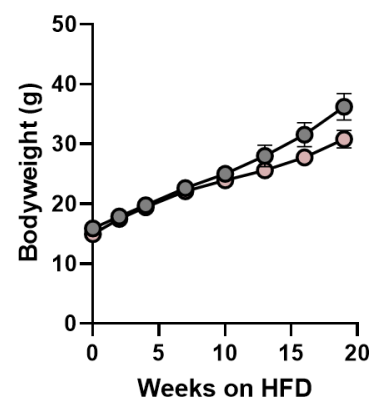

C

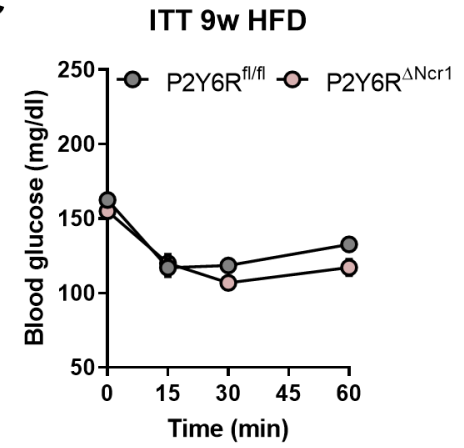

$\mathbf{F}$

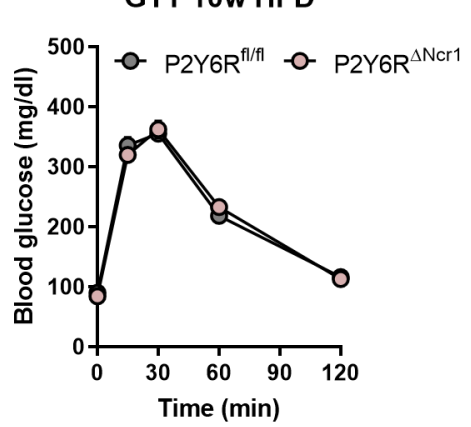

B

\section{Organ weights}

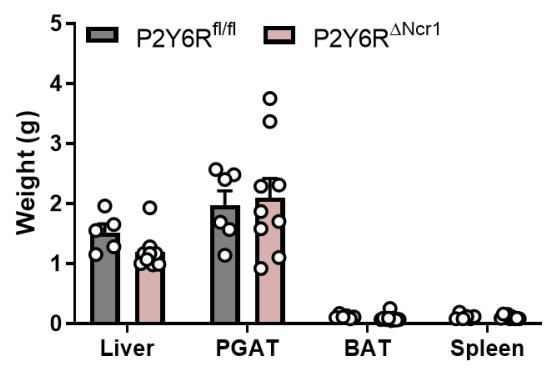

D

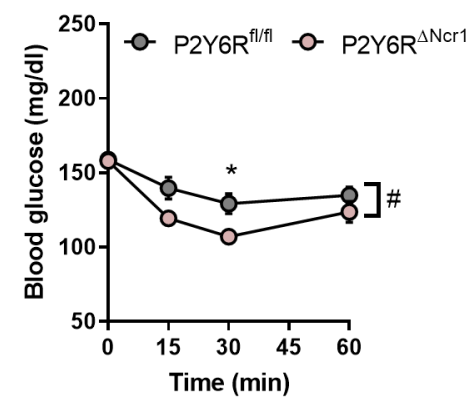

G

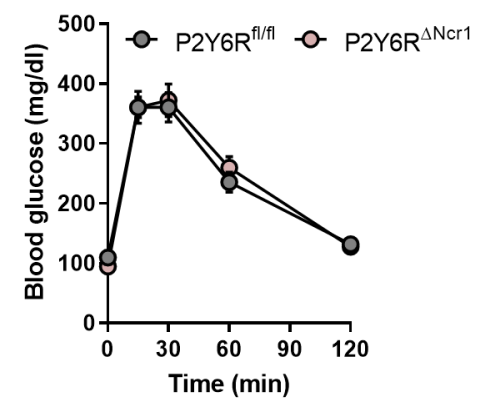

E

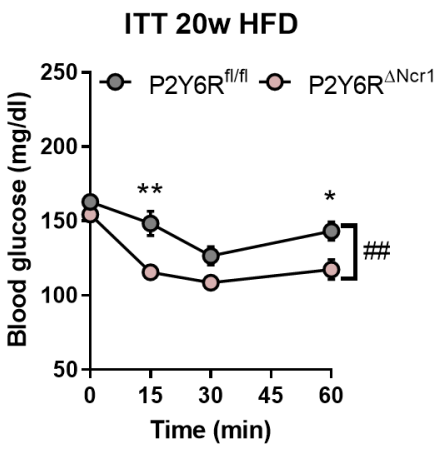

H

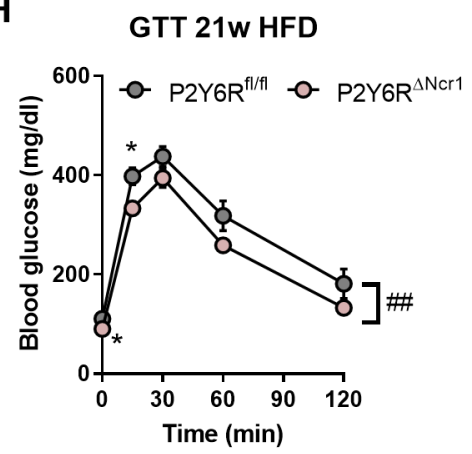


A

Food Intake

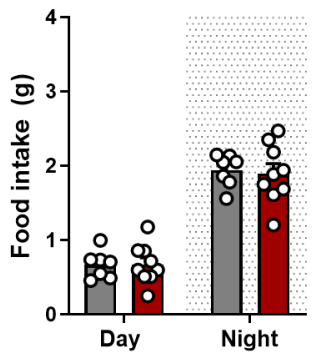

C

Energy Expenditure

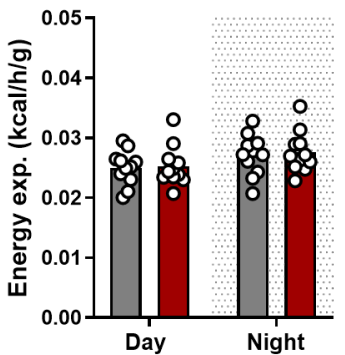

B Water Consumption

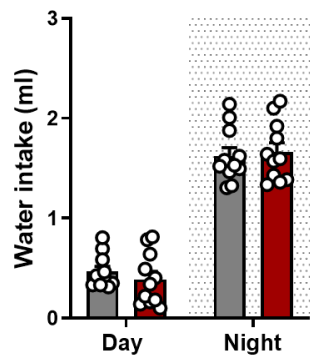

D

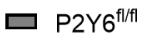

- $\mathrm{P}_{2} \mathrm{Y}^{\mathrm{AN} \mathrm{N} 1}$

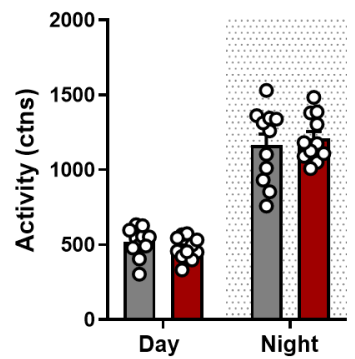

口 $\mathrm{P} 2 \mathrm{YG}^{\mathrm{fl/fI}}$

$\square \mathrm{P}_{2} \mathrm{Y6}^{\mathrm{\Delta N} \mathrm{N} 1}$ 
A Body weight development

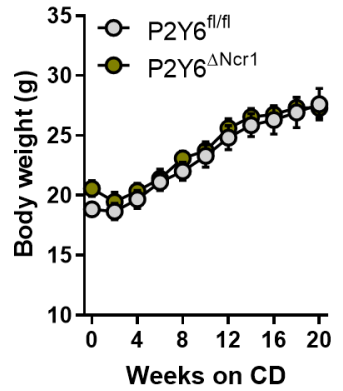

C

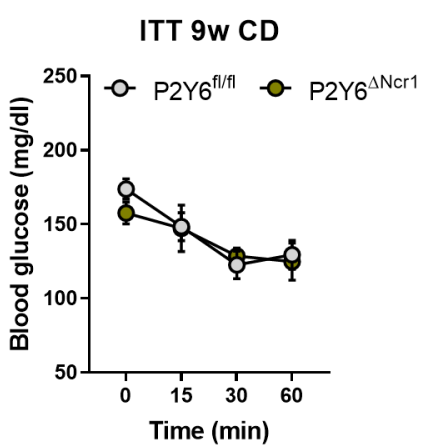

F

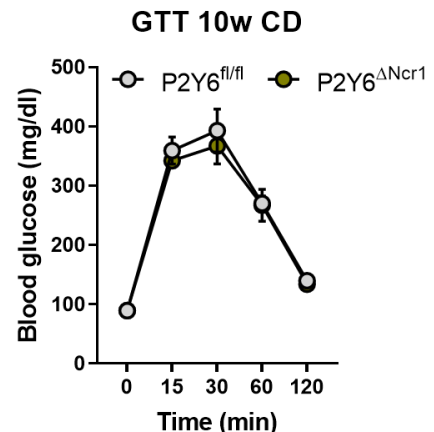

I

Insulin

10w CD

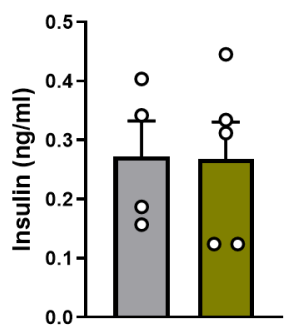

B

Organ weights

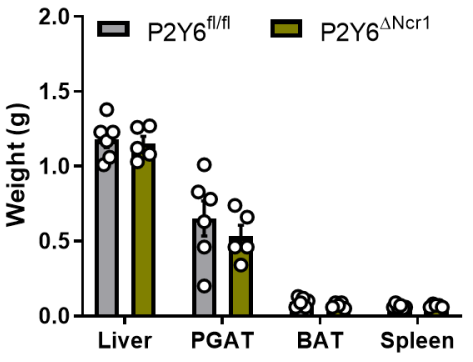

D

ITT 13w CD

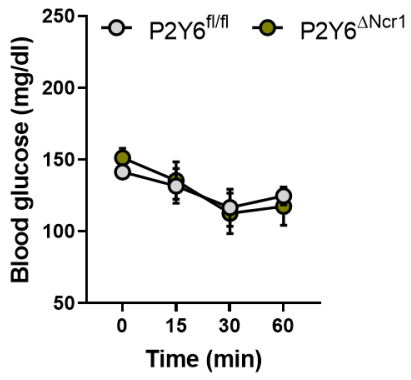

G

GTT 14w CD

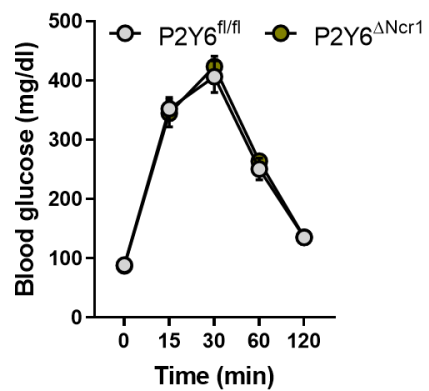

H

E ITT 20w CD

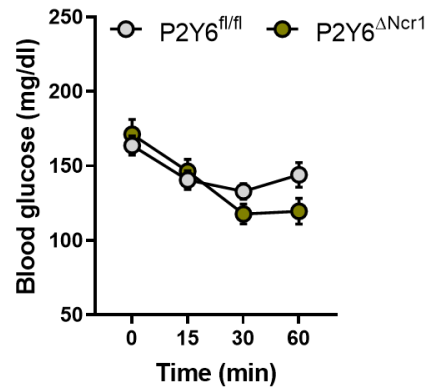

GTT 21w CD

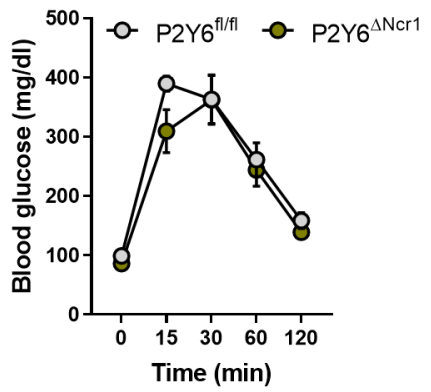

J

HOMA-IR

10W CD

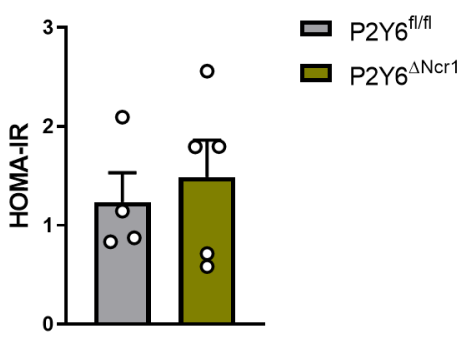




\section{Clamped PGAT}

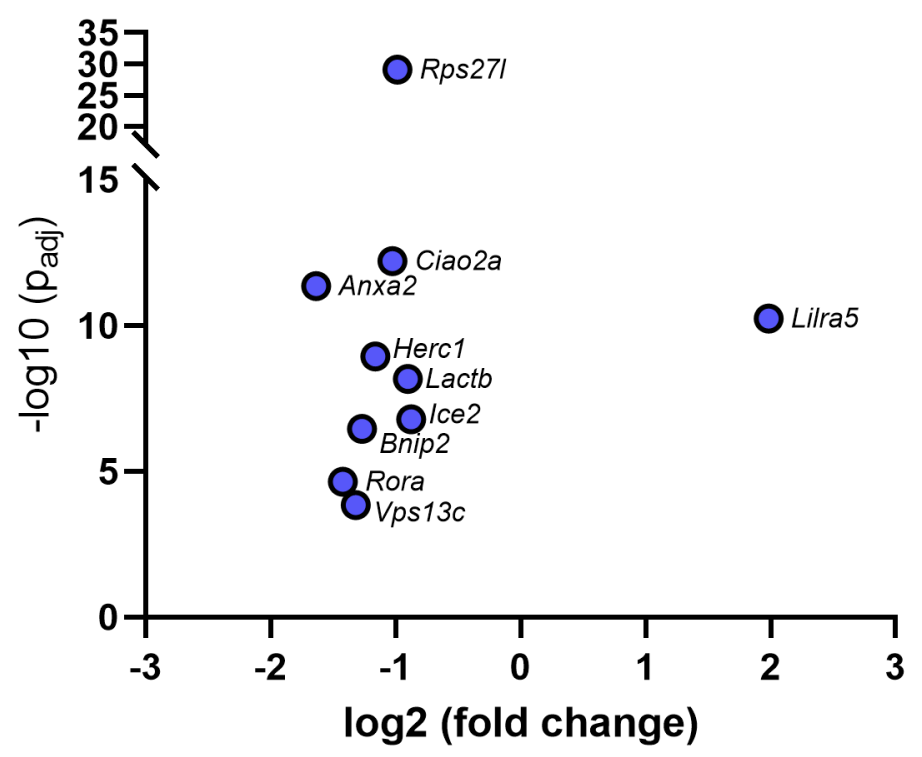

\section{Clamped Liver}

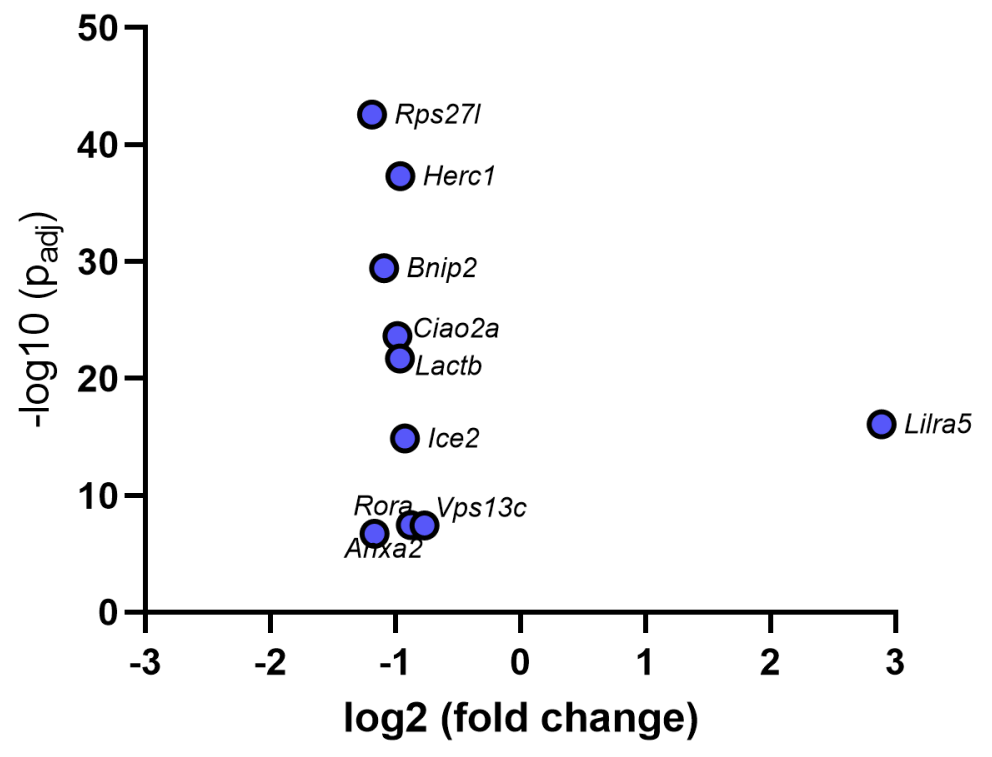


A

MAC2 Staining PGAT

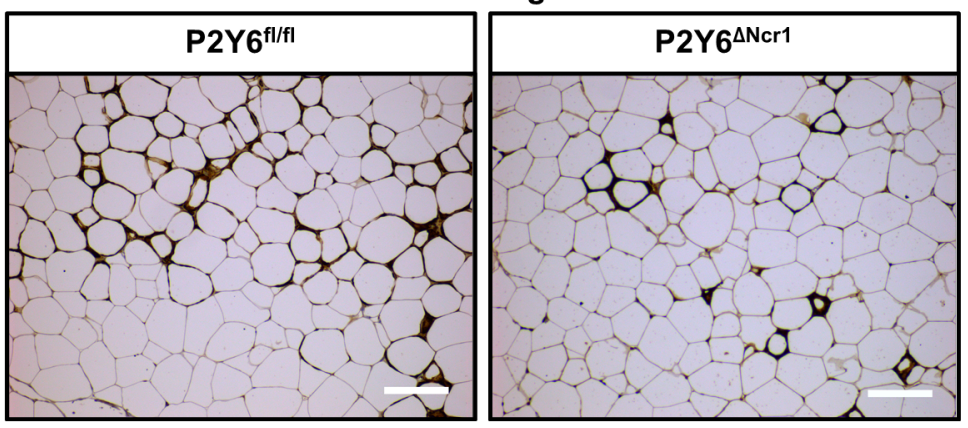

MAC2

Adipocyte cell size

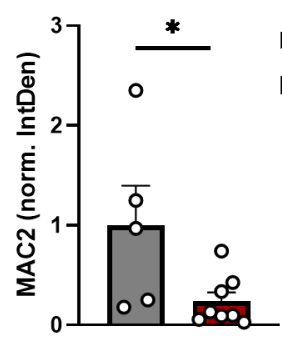

B

Inflammatory Markers PGAT

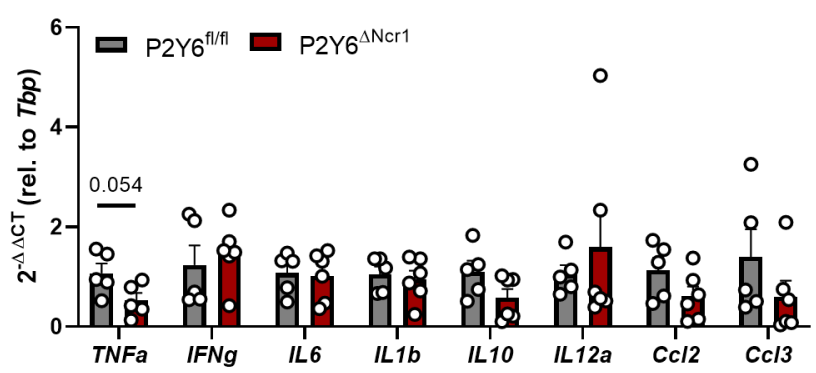

C

Inflammatory Markers Liver

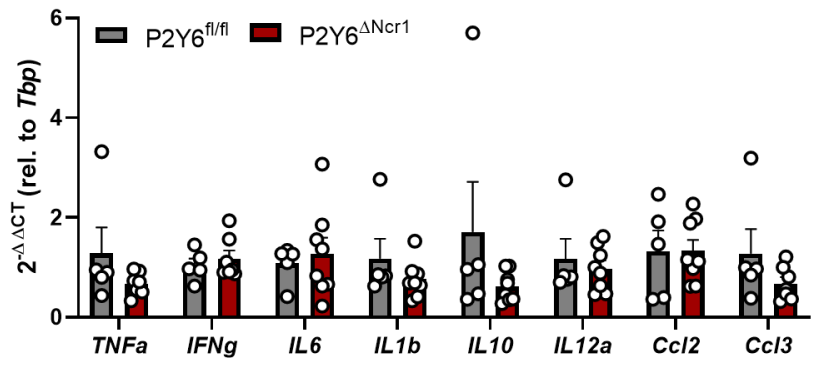


A

A P2y6r

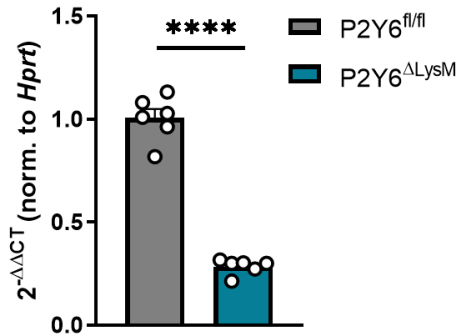

C
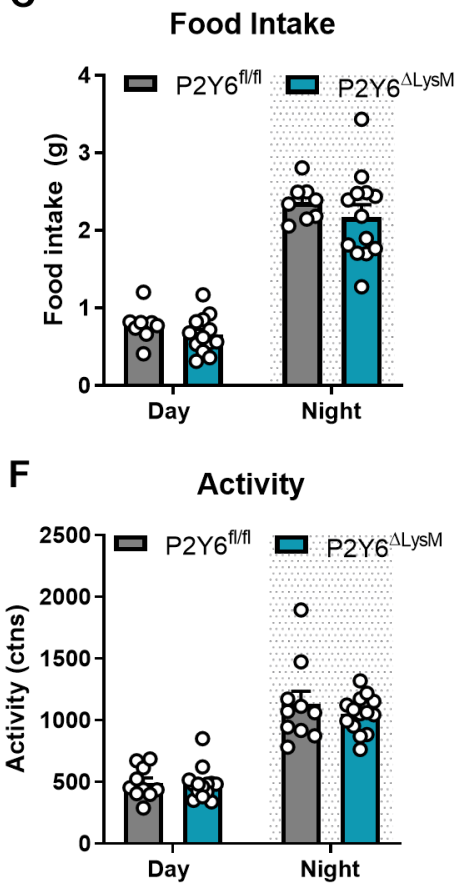

B

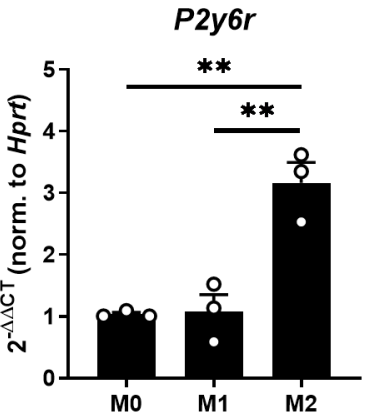

Macrophage Polarization

D

Water Consumption

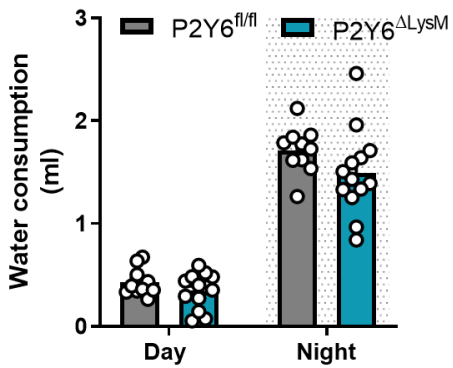

G

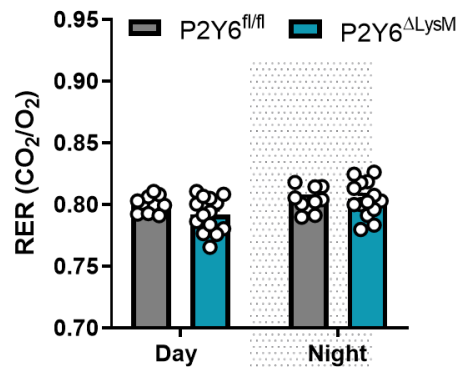

E

Energy Expenditure

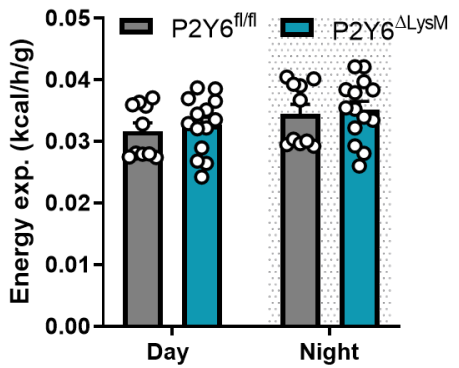




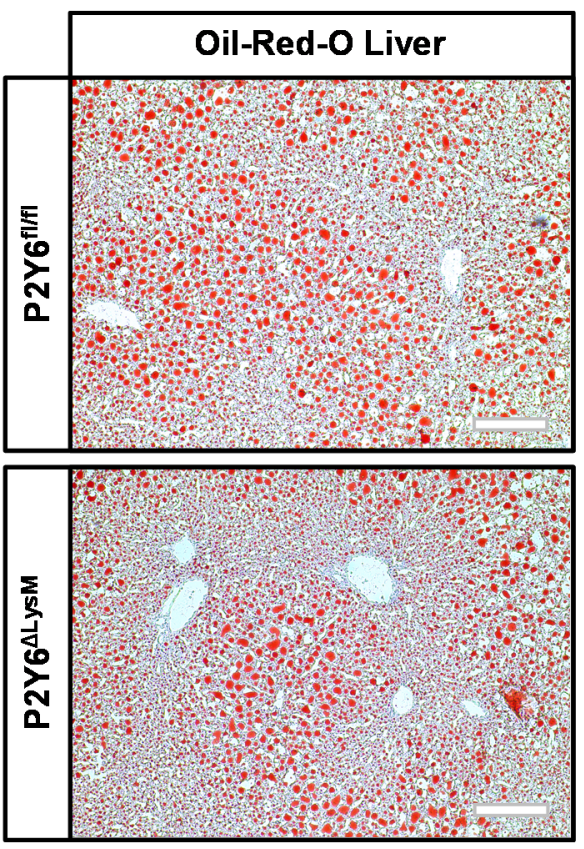

Oil-Red-O Liver

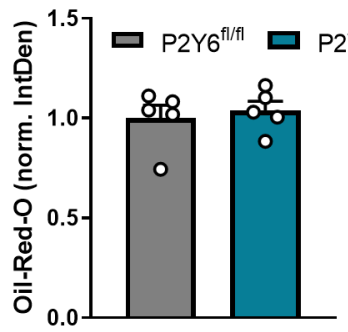

\section{MAC2 PGAT}
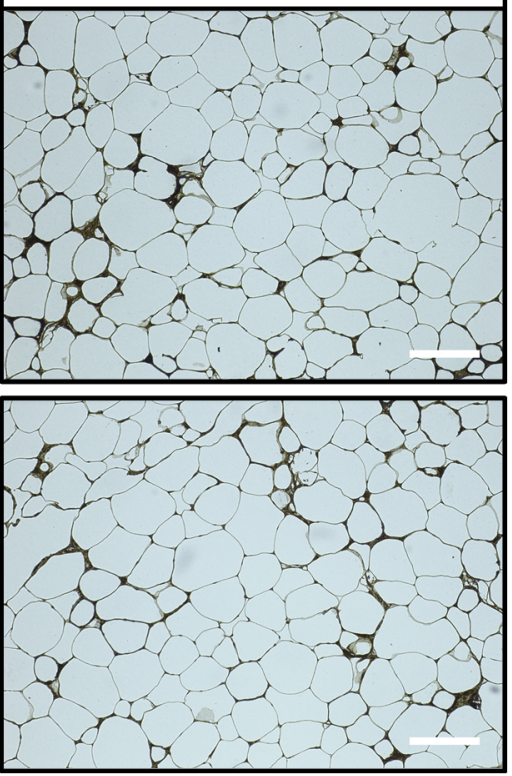

MAC2 PGAT

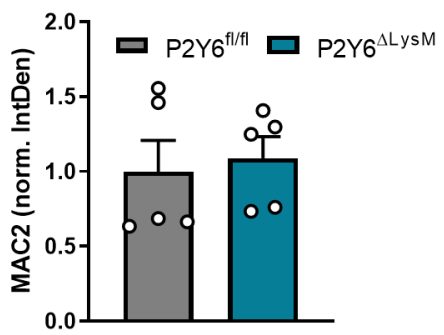

